\title{
Spectral Sequences and Adiabatic Limits
}

\author{
Robin Forman ${ }^{\star}$ \\ Department of Mathematics, Rice University, Houston, TX 77251
}

Received: 29 November 1993

Abstract: A Taylor series analysis of the Laplacian as the underlying manifold is deformed leads to a Hodge theoretic derivation of the Leray spectral sequence.

\section{Introduction}

Suppose $(M, g)$ is a compact Riemannian manifold with a smooth distribution of $n$-planes $A$. Let $B$ be the orthogonal distribution to $A$. Writing

$$
g=g_{A} \oplus g_{B},
$$

we define a 1-parameter family of metrics on $M$ by setting, for $0<\delta \leqq 1$,

$$
g_{\delta}=g_{A} \oplus \delta^{-2} g_{B} .
$$

In addition, let

$$
V \rightarrow M
$$

be a flat bundle,

In this paper we investigate a spectral sequence associated with $A$ and $B$ for the cohomology of $M$ with values in $V$. We show in Sects. 2 and 3 how the spectral sequence arises naturally from a Taylor series analysis of the eigenvalues of $\square_{\delta}^{p}$ near $\delta=0$ (where $\square_{\delta}^{p}$ denotes the Laplacian induced by the metric $g_{\delta}$ acting on $p$-forms of $M$ with values in $V$ ). We demonstrate how the algebraic properties of the spectral sequence can be proved using standard Hodge theory. In Sect. 4 we show that our spectral sequence is intimately related to the Leray spectral sequence associated to a filtered differential complex. In addition, of $A$ is integrable, the spectral sequence is isomorphic to the standard Leray spectral sequence associated to the foliation $A$. If $A$ is integrable, and in addition satisfies certain geometric restrictions (see hypotheses (H1) and (H2)), we show in Sect. 5 that the leading order asymptotics of the small eigenvalues of $\square_{\delta}^{p}$, and the corresponding eigenspaces, are determined by

$\star$ Partially supported by an NSF postdoctoral fellowship 
information contained in the spectral sequence. In the special case that the splitting of $T M$ arises from a fibration, our results overlap with those of [Ma-Me] and [Dai].

The limit of $\left(M, g_{\delta}\right)$ as $\delta \rightarrow 0$ is known as the "adiabatic limit." The adiabatic limit was introduced in this form by Witten in [Wi]. He considered the foliation $F$ consisting of the fibers of a fibration

$$
\mathscr{F} \hookrightarrow M \rightarrow N,
$$

where $\mathscr{F}$ is compact, $N=S^{1}$, and the metric $g$ makes $(0.1)$ a Riemannian submersion. Witten investigated the limit of the eta-invariant of $M$ and $\delta$ approached 0 . This question was also considered in [Bi-Fr] and [Ch]. In [Bi-Ch] and [Dai], this investigation was extended to general base spaces $N$.

Our topic begins with (and this paper owes much to the ideas in) [Ma-Me] in which the authors, starting with a fibration as in (0.1), analyse the behavior of the space of harmonic forms on $M$ as $\delta \rightarrow 0$. They show that, modulo a change of coordinates, the space of harmonic $p$-forms approaches a finite dimensional space $\bar{E}_{\infty}^{p}$. This space $\bar{E}_{\infty}^{p}$ can be identified from a Taylor series analysis as follows: Define a nested family of spaces

$$
\bar{E}_{0}^{p} \supseteq \bar{E}_{1}^{p} \supseteq \bar{E}_{2}^{p} \supseteq \cdots
$$

by

$$
\begin{aligned}
\bar{E}_{k}^{p}= & \left\{p \text {-forms } \omega \mid \exists p \text {-forms } \omega_{1}, \ldots, \omega_{j}\right. \text { with } \\
& \left.\square_{\delta}^{p}\left(\omega+\delta \omega_{1}+\cdots+\delta^{j} \omega^{j}\right) \in 0\left(\delta^{k}\right)\right\} .
\end{aligned}
$$

They proved that there is an $N$ such that

$$
\bar{E}_{N}^{p}=\bar{E}_{N+1}^{p}=\cdots=\bar{E}_{\infty}^{p},
$$

and this is the space referred to above. These results follow from their construction, using Melrose's calculus of pseudodifferential operators on manifolds with corners, of a parametrix for $\square_{\delta}^{p}$ which has a uniform extension to the closed interval $[0,1]$. This implies that in the case of a fibration, the eigenvalues and eigenvectors have well-defined asymptotics as $\delta \rightarrow 0$.

In this paper, we take a simpler, and more general approach. We begin with any distribution. $A \subset T M$. In particular, we do not require that $A$ arise from a fibration, or even that $A$ be integrable. We show that a Taylor series analysis, motivated by the adiabatic limit, leads directly to a Hodge theoretic spectral sequence which converges to the cohomology of $M$. We observe that the spectral sequence structure, i.e. the associated differentials and bigradings (which do not appear in [Ma-Me]), arise naturally in our context.

We start with a rescaling map $\rho_{\delta}$ (see Lemma 1.2), which also appears implicitly in $[\mathrm{Ma}-\mathrm{Me}]$, which is an isometry

$$
\rho_{\delta}:\left(\Omega^{p}(V), g_{\delta}\right) \rightarrow\left(\Omega^{p}(V), g\right)
$$

(where $\Omega^{p}(V)$ denotes $p$-forms on $M$ with values in $V$ ). Let

$$
d_{\delta}=\rho_{\delta} d \rho_{\delta}^{-1}, \quad d_{\delta}^{*}=\rho_{\delta} \dot{d}_{\left(g_{\delta}\right)}^{*} \rho_{\delta}^{-1},
$$

and define a nested sequences of spaces

$$
E_{-1} \supseteq E_{0}^{p} \supseteq E_{1}^{p} \supseteq E_{2}^{p} \supseteq \ldots
$$


by

$$
\begin{aligned}
E_{k}^{p}= & \left\{\omega \in \Omega^{p}(M, V) \mid \exists \omega_{1}, \ldots, \omega_{j}\right. \text { with } \\
& d_{\delta}\left(\omega+\delta \omega_{1}+\cdots+\delta^{j} \omega^{j}\right) \in 0\left(\delta^{k}\right) \\
& \left.d_{\delta}^{*}\left(\omega+\delta \omega_{1}+\cdots+\delta^{j} \omega_{j}\right) \in 0\left(\delta^{k}\right)\right\} .
\end{aligned}
$$

Our first result (Theorem 1.3) is that the sequence $(0.2)$ converges. That is, there is an $N$ such that

$$
E_{N}^{p}=E_{N+1}^{p}=\cdots=E_{\infty}^{p}
$$

and, in fact, $\operatorname{dim} E_{N}^{p}<\infty$. More precisely, we show (Theorem 1.3) that $\operatorname{dim} E_{N^{\prime}}^{p}<$ $\infty$, where

$$
N^{\prime}=\min \{\operatorname{dim} A+3, \operatorname{dim} B+3\} .
$$

We define a differential $d_{k}$ on $E_{k}^{p}$ by setting, for $\omega \in E_{k}^{p}$,

$$
d_{k} \omega=\lim _{\delta \rightarrow 0} \delta^{-k} d_{\delta}\left(w+\delta w_{1}+\cdots+\delta^{j} \omega_{j}\right),
$$

where the $\omega_{i}$ 's are as in (0.3). Unfortunately, the map $d_{k}$ depends on the $\omega_{i}$ 's, but the map $\pi_{k} d_{k}$ does not, where $\pi_{k}$ denotes the orthogonal projection onto $E_{k}^{p}$. In fact we show (Theorem 2.3)

(i) $\left(\pi_{k} d_{k} \pi_{k}\right)^{2}=0$.

(ii) The kernel of

$$
\Delta_{k}=\left(\pi_{k} d_{k} \pi_{k}\right)\left(\pi_{k} d_{k}^{*} \pi_{k}\right)+\left(\pi_{k} d_{k}^{*} \pi_{k}\right)\left(\pi_{k} d_{k} \pi_{k}\right): E_{k}^{p} \rightarrow E_{k}^{p}
$$

is precisely $E_{k+1}^{p}$.

Statement (i) says that for each $k$

$$
\pi_{k} d_{k} \pi_{k}: E_{k}^{0} \rightarrow E_{k}^{1} \rightarrow E_{k}^{2} \rightarrow \cdots
$$

forms a differential complex, and (ii) implies that the cohomology of the complex at the $p^{\text {th }}$ stage is isomorphic to $E_{k+1}^{p}$. (There are some analytical subtleties here, but this is certainly true if $\operatorname{dim} E_{k}^{p}<\infty$, for example if $k \geqq N^{\prime}$.) Thus the complexes $\left\{E_{k}^{p}, \pi_{k} d_{k} \pi_{k}\right\}$ form a spectral sequence.

Before leaving Sect. 2, we prove that the $E_{k}^{p}$ spaces inherit a natural bigrading from the decomposition $T M=A+B$ which is compatible with the differential $\pi_{k} d_{k} \pi_{k}$. That is,

$$
E_{k}^{p}=\bigoplus_{a+b=p} E_{k}^{a, b}, \text { where } E_{k}^{a, b}=E_{k}^{p} \cap \Omega^{a, b}
$$

and

$$
\pi_{k} d_{k} \pi_{k}\left(E_{k}^{a, b}\right) \subseteq E_{k}^{a-k+1, b+k} .
$$

In Sect. 3 we make precise the sense in which (0.2) converges to $H^{p}(M, V)$, the cohomology of $M$ with values in $V$. The main idea is to introduce the cohomology of the space of formal Laurent series of forms on $M$. This section essentially follows the ideas of Sects. 2, 5 and 6 of [Ma-Me], albeit in a more general setting. The conclusions follow from these observations: 
(i) For every $\omega \in E_{\infty}^{p}$ there is a formal power series

$$
\omega_{\delta}=\omega+\delta \omega_{1}+\delta^{2} \omega_{2}+\cdots
$$

such that, formally,

$$
d_{\delta} \omega_{\delta}=d_{\delta}^{*} \omega_{\delta}=0 .
$$

(ii) The $\omega_{\delta}$ 's arising in (i) form a basis, modulo the action of $\mathscr{L}$ (the ring of formal real Laurent series), for the cohomology of the complex $\left(\mathscr{L}\left[\Omega^{p}\right], d_{\delta}\right)$. Here, $\mathscr{L}\left[\Omega^{p}\right]$ denotes the space of formal Laurent series with coefficients in $\Omega^{p}(M, V)$.

(iii) The operator $\rho_{\delta}$ provides an isomorphism between $\left(\mathscr{L}\left[\Omega^{p}\right], d_{\delta}\right)$ and $\left(\mathscr{L}\left[\Omega^{p}\right], d\right)$.

(iv) The cohomology of $\left(\mathscr{L}\left[\Omega^{p}\right], d\right)$ is canonically isomorphic to $\mathscr{L}\left[H^{p}(M, V)\right]$ and hence, modulo $\mathscr{L}, H^{p}(M, V)$ provides a basis

Observations (i)-(iv) allow us to conclude, in particular, that for all $p$,

$$
\operatorname{dim} E_{\infty}^{p}=\operatorname{dim} H^{p}(M, V) .
$$

The topological nature of the spaces $E_{k}^{p}$ is clarified in Sect. 4. We demonstrate that the spectral sequence $\left\{\mathscr{L}\left[E_{k}^{p}\right], \pi_{k} d_{k} \pi_{k}\right\}$ is isomorphic to the Leray spectral sequence arising from the natural infinite filtration of $\mathscr{L}\left[\Omega^{p}\right]$ :

$$
\mathscr{L}\left[\Omega^{p}\right]=\cdots \supseteq \delta^{-1} \Omega^{p}[[\delta]] \supseteq \Omega^{p}[[\delta]] \supseteq \delta \Omega^{p}[[\delta]] \supseteq \cdots
$$

and the differential $\partial=\delta d_{\delta}$. As a corollary, we learn that the dimensions of the $E_{k}^{p}$ are independent of the metrics $g_{A}$ and $g_{B}$ (since the metrics are not used in the construction of the Leray spectral sequence). In addition, if $A$ is integrable we show that the spectral sequence $\left\{E_{k}^{p}, \pi_{k} d_{k} \pi_{k}\right\}$ is isomorphic to the standard Leray spectral sequence associated to the foliation $A$. In this case we learn that $\operatorname{dim} E_{k}^{p}$ depends only on $A$, that is, it is independent of the chosen complement $B$ as well as the metrics $g_{A}$ and $g_{B}$. In the special case of a fibration, this was proved in [Dai].

We make the relationship between $E_{\infty}^{p}$ and $H^{p}(M, V)$ more direct in Sect. 5. In this section we require, essentially, that $A$ be a Riemannian foliation with compact leaves, and that $g$ be a bundle-like metric. In particular, we require that $(M, A, g)$ be given locally, by a fibration of the type $(0.1)$. However, there are many interesting examples of foliations satisfying our hypotheses which are not globally of the form (0.1). We note that these restrictions are required only for the analysis of this section.

We study the behavior of the small eigenvalues of $\square_{\delta}^{p}$ and the corresponding eigenspaces. Let

$$
\lambda_{1}^{p}(\delta) \leqq \lambda_{2}^{p}(\delta) \leqq \cdots
$$

denote the eigenvalues of $\square_{\delta}^{p}$. Let

$$
\operatorname{eig}_{k}^{p}=\operatorname{span}\left\{\omega \in \Omega^{p} \mid \rho_{\delta} \square_{\delta}^{p} \rho_{\delta}^{-1} \omega=\lambda_{i}^{p}(\delta) \omega \text { and } \lambda_{i}^{p}(\delta) \in 0\left(\delta^{2 k}\right)\right\}
$$

Then we show (Theorem 5.17) that as $\delta \rightarrow 0$,

$$
\operatorname{eig}_{k}^{p}=E_{k}^{p}+0(\delta) \text {. }
$$

As a corollary, if $\mathscr{H}_{\delta}^{p}(M, V)$ denotes the kernel of $\square_{\delta}^{p}$, then as $\delta \rightarrow 0$, 


$$
\rho_{\delta} \mathscr{H}_{\delta}^{p}(M, V)=E_{\infty}^{p}+0(\delta) .
$$

Now write $\lambda_{i}^{p}(\delta) \sim \delta^{k}$ if there is a $c$ such that for all $\delta \in(0,1)$,

$$
c \delta^{k}<\lambda_{l}^{p}(\delta)<\frac{1}{c} \delta^{k}
$$

Then every $\lambda_{i}^{p}(\delta)$ is $\sim \delta^{2 k}$ for some $k$. The leading order term of such $\lambda_{l}^{p}$ is determined by the Taylor series data. Namely, let $\widetilde{E}_{k}^{p}$ denote the orthogonal complement of $E_{k+1}^{p}$ in $E_{k}^{p}$. We have seen that the kernel of

$$
\Delta_{k}^{p}: E_{k}^{p} \rightarrow E_{k}^{p}
$$

is $E_{k+1}^{p}$. Since $\Delta_{k}^{p}$ is self-adjoint, $\Delta_{k}^{p}$ must map $\widetilde{E}_{k}^{p}$ to itself. We have for $k \geqq 1$ (Theorem 5.20)

$$
\left\{\lambda_{i}^{p}(\delta) \mid \lambda_{i}^{p}(\delta) \sim \delta^{2 k}\right\}=\delta^{2 k}\left\{\text { eigenvalues of } \Delta_{k}^{p}: \widetilde{E}_{k}^{p} \rightarrow \widetilde{E}_{k}^{p}\right\}+0\left(\delta^{2 k+1}\right) .
$$

The statement (0.4) implies, in particular, that the one-parameter family of spaces $\rho_{\delta} \mathscr{H}_{\delta}^{p}, \delta \in(0,1]$ has a continuous extension to the closed interval $\delta \in[0,1]$. We complete Sect. 5 by sharpening this statement. Namely, we show that a slight modification of our proof of (0.4) yields (Theorem 5.21) that this extension is, in fact, $C^{\infty}$ on $[0,1]$. Equivalently, if

$$
\omega_{\delta}=\omega+\delta \omega_{1}+\delta^{2} \omega_{2}+\cdots
$$

is any formal power series in $\delta$ with values in $\Omega^{p}(M, V)$, which formally satisfies

$$
L_{\delta}^{p} \omega_{\delta}=0
$$

then $\omega_{\delta}$ is the Taylor series at $\delta=0$ of a $C^{\infty}$ family of forms $\tilde{\omega}_{\delta}, \delta \in[0,1]$, which satisfy, for each $\delta \in(0,1]$,

$$
L_{\delta}^{p} \omega_{\delta}=0 \text {. }
$$

This extends Theorem 17 of [Ma-Me] to our setting.

In the special case of a fibration, the results in Sect. 5 overlap with those of [Dai] and [Ma-Me]. In [Dai], the results of [Ma-Me] were used to study the adiabatic limit of the eta-invariant of the fiber bundle $M$. In an analogous fashion, in another paper we will use these results to derive a formula for the analytic torsion of $M$.

In Sects. 1-4, we show that the adiabatic limit leads to a natural spectral sequence for any splitting of the tangent space $T M=A+B$. It remains an intriguing problem to find a more general context for the analysis of Sect. 5. That is, less restrictive geometric assumptions which still imply that the spectral data determines the precise asymptotics of the eigenvalues and eigenspaces.

\section{Preliminaries}

Let $\left(M^{m}, g\right)$ be a compact Riemannian manifold, and $V \rightarrow M$ a flat vector bundle. That is, $V$ comes equipped with a Euclidean inner product and a compatible flat connection. By $\Omega^{p}(V)$ we denote the space of $p$-forms on $M$ with values in $V$. There is a natural extension of the usual deRham differential to a differential 


$$
d: \Omega^{p}(V) \rightarrow \Omega^{p+1}(V) .
$$

Suppose $A \subset T M$ is a smooth distribution of $n$-planes, and $B=A^{\perp}$ is the orthogonal distribution. Then we have a decomposition

$$
T M=A \oplus B
$$

and a corresponding decomposition

$$
T^{*} M=A^{*} \oplus B^{*} .
$$

This decomposition induces a bigrading on $\Omega^{p}(V)$ by

$$
\Omega^{p}(V)=\bigoplus_{i=0}^{p} \Omega^{i, p-i}(V),
$$

where

$$
\Omega^{i, p-i}(V)=\Gamma\left(\Lambda^{i} A^{*} \oplus \Lambda^{j} B^{*} \oplus V\right) .
$$

Similarly, all operators on forms inherit a corresponding decomposition. In particular, the $d$ operator inherits a bigrading

$$
d=\sum_{i} d^{i, l-i}
$$

where

$$
d^{a, b}: \Omega^{i, j}(V) \rightarrow \Omega^{i+a, j+b}(V) .
$$

For any such decomposition of $T M$ we have

$$
d=d^{2,-1}+d^{1,0}+d^{0,1}+d^{-1,2} .
$$

Note that $d^{1,0}$ and $d^{0,1}$ are first order differential operators, while $d^{2,-1}$ and $d^{-1,2}$ are zeroth order. Geometric properties of the distributions $A$ and $B$ are reflected in the analytic properties of these operators. For example

Lemma 1.1. The operator $d^{2,-1}=0$ if and only if $A$ is integrable

For a proof, see [Mo], page 58 .

The identity $d^{2}=0$ yields the identities

$$
\begin{aligned}
& 0=\left(d^{2}\right)^{4,-2}=\left(d^{2,-1}\right)^{2} \\
& 0=\left(d^{2}\right)^{3,-1}=d^{2,-1} d^{1,0}+d^{1,0} d^{2,-1} \\
& 0=\left(d^{2}\right)^{2,0}=d^{2,-1} d^{0,1}+\left(d^{1,0}\right)^{2}+d^{0,1} d^{2,-1} \\
& 0=\left(d^{2}\right)^{1,1}=d^{2,-1} d^{-1,2}+d^{1,0} d^{0,1}+d^{0,1} d^{1,0}+d^{-1,2} d^{2,-1} \\
& 0=\left(d^{2}\right)^{0,2}=d^{1,0} d^{-1,2}+\left(d^{0,1}\right)^{2}+d^{-1,2} d^{1,0} \\
& \left.0=\left(d^{2}\right)^{-1,3}=d^{0,1} d^{-1,2}+d^{-1,2}\right) d^{0,1} \\
& 0=\left(d^{2}\right)^{-2,4}=\left(d^{-1,2}\right)^{2} .
\end{aligned}
$$

The decomposition of the tangent space (1.1) induces a corresponding decomposition of the metric

$$
g=g_{A} \oplus g_{B} .
$$

We define a 1 -parameter family of metrics $g_{\delta}, 0<\delta \leqq 1$, by 


$$
g_{\delta}=g_{A} \oplus \delta^{-2} g_{B}
$$

For each $p$ and $\delta$ we have an induced Lapalcian

$$
\square_{\delta}^{p}=d_{\left(g_{\delta}\right)}^{*} d+d d_{\left(g_{\delta}\right)}^{*}: \Omega^{p}(V) \rightarrow \Omega^{p}(V),
$$

where $d_{\left(g_{\delta}\right)}^{*}$ is the adjoint of $d$ with respect to the metric on $\Lambda^{*} T^{*} M$ induced by the metric $g_{\delta}$.

Our goal is to study the behavior of the eigenvalues of $\square_{\delta}^{p}$ as $\delta \rightarrow 0$. In our investigation, we will make use of the classical variational approach (see [Du-Sc], p. 908)

$$
\lambda_{i}^{p}(\delta)=\sup _{v_{1}, \ldots, v_{i-1} \in H_{1}^{p}} \inf _{\substack{v_{1} \in H_{1}^{p} \\ v_{i} \perp\left\{v_{1}, \ldots, v_{i-1}\right\}}} \frac{\left\langle d v_{i}, d v_{i}\right\rangle_{\delta}+\left\langle d_{\left(g_{\delta}\right)}^{*} v_{1}, d_{\left(g_{\delta}\right)}^{*} v_{i}\right\rangle_{\delta}}{\left\langle v_{l}, v_{i}\right\rangle_{\delta}}
$$

Here we have numbered the eigenvalues of $\square_{\delta}^{p}$ in increasing order, with each eigenvalue listed according to its multiplicity:

$$
\lambda_{1}^{p}(\delta) \leqq \lambda_{2}^{p}(\delta) \leqq \cdots
$$

Moreover, $H_{1}^{p}$ denotes the completion, in the space of $L^{2} p$-forms, of the $C^{\infty} p$ forms with respect to the norm

$$
\|\omega\|_{H_{1}}=\|\nabla \omega\|_{L^{2}}^{2}+\|\omega\|_{L^{2}}^{2} .
$$

Note that the space $H_{1}^{p}$ is independent of the metric used to define the norms, and thus is independent of $\delta$.

One difficulty in applying (1.2) is that both the operator and the inner product vary with $\delta$. To simplify, we introduce the isometry

$$
\rho_{\delta}:\left(\Omega^{p}(V), g_{\delta}\right) \rightarrow\left(\Omega^{p}(V), g\right),
$$

where, for $\omega \in \Omega^{l, J}$,

$$
\rho_{\delta} \omega=\delta^{J} \omega
$$

Then for all $\omega \in \Omega^{p}$,

$$
\frac{\left\langle\square_{\delta}^{p} \omega, \omega\right\rangle_{\delta}}{\langle\omega, \omega\rangle_{\delta}}=\frac{\left\langle\left(\rho_{\delta} \square_{\delta}^{p} \rho_{\delta}^{-1}\right)(\rho \delta \omega),(\rho \delta \omega)\right\rangle}{\langle\rho \delta \omega, \rho \delta \omega\rangle},
$$

where we use $\langle$,$\rangle to denote the inner product induced by the original metric g$.

Lemma 1.2. Let $L_{\delta}^{p}=\rho_{\delta} \square_{\delta}^{p} \rho_{\delta}^{-1}$. Then

$$
L_{\delta}^{p}=d_{\delta} d_{\delta}^{*}+d_{\delta}^{*} d_{\delta}
$$

where

$$
d_{\delta}=\delta^{-1} d^{2,-1}+d^{1,0}+\delta d^{0,1}+\delta^{2} d^{-1,2}
$$

and

$$
d_{\delta}^{*}=\delta^{-1}\left(d^{2,-1}\right)^{*}+\left(d^{1,0}\right)^{*}+\delta\left(d^{0,1}\right)^{*}+\delta^{2}\left(d^{-1,2}\right)^{*}
$$

is the adjoint. Note that all adjoints are taken with respect to the metric $g$.

Proof. First note that 


$$
L_{\delta}^{p}=\left(\rho_{\delta} d \rho_{\delta}^{-1}\right)\left(\rho_{\delta} d_{\left(g_{\delta}\right)}^{*} \rho_{\delta}^{-1}\right)+\left(\rho_{\delta} d_{\left(g_{\delta}\right)}^{*} \rho_{\delta}^{-1}\right)\left(\rho_{\delta} d \rho_{\delta}^{-1}\right) .
$$

The operator $\rho_{\delta} d_{\left(g_{\delta}\right)}^{*} \rho_{\delta}^{-1}$ is the adjoint of $\rho_{\delta} d \rho_{\delta}^{-1}$ with respect to the metric $g$, so (1.4) follows from (1.3). To prove (1.3) note that for all $\omega \in \Omega^{i, j}$,

$$
\rho_{\delta} d^{a, b} \rho_{\delta}^{-1} \omega=\rho_{\delta} d^{a, b} \delta^{-j} \omega=\delta^{-j} \rho_{\delta}(d \omega)^{i+a, j+b}=\delta^{-j} \delta^{j+b}(d \omega)^{i+a, j+b}=\delta^{b} d^{a, b} \omega .
$$

Thus, if we write

$$
d=\sum d^{a, b}
$$

then

$$
\rho_{\delta} d \rho_{\delta}^{-1}=\sum \delta^{b} d^{a, b} .
$$

Using Lemma 1.2 we can reformulate (1.2) as

$$
\lambda_{i}^{p}(\delta)=\sup _{v_{1}, \ldots, v_{i-1} \in H_{1}^{p}} \inf _{\substack{v_{i} \in H_{1} \\ v_{i} \perp\left\{v_{1}, \ldots, v_{i-1}\right\}}} \frac{\left|d_{\delta} v_{i}\right|^{2}+\left|d_{\delta}^{*} v_{i}\right|^{2}}{\left|v_{i}\right|^{2}} .
$$

This quotient motivates the following definition. Define the nested sequence of spaces

$$
E_{-1}^{P} \supseteq E_{0}^{p} \supseteq E_{1}^{p} \supseteq E_{2}^{p} \supseteq \cdots .
$$

where

$$
\begin{aligned}
E_{k}^{p}= & \left\{\omega \in H_{1}^{p} \mid \exists j \text { and } \omega_{1}, \ldots, \omega_{j} \in H_{1}^{p}\right. \text { with } \\
& d_{\delta}\left(\omega+\delta \omega_{1}+\cdots+\delta^{j} \omega_{j}\right) \in \delta^{k} \Omega^{p+1}[\delta] \\
& \left.d_{\delta}^{*}\left(\omega+\delta \omega_{1}+\cdots+\delta^{j} \omega_{j}\right) \in \delta^{k} \Omega^{p-1}[\delta]\right\},
\end{aligned}
$$

where $\Omega^{i}[\delta]$ denotes the space of polynomials with $\delta$ with coefficients in $\Omega^{i}(E)$.

Example. For $\omega_{\delta}=\omega+\delta \omega_{1}+\cdots+\delta^{j} \omega_{J}$,

$$
\begin{aligned}
& d_{\delta} \omega_{\delta}=\delta^{-1} d^{2,-1} \omega+\left(d^{1,0} \omega+d^{2,-1} \omega_{1}\right)+\cdots, \\
& d_{\delta}^{*} \omega_{\delta}=\delta^{-1}\left(d^{2,-1}\right)^{*} \omega+\left(\left(d^{1,0}\right)^{*} \omega+\left(d^{2,-1}\right)^{*} \omega_{1}\right)+\cdots .
\end{aligned}
$$

Therefore

$$
\begin{aligned}
& E_{0}^{p}=\left\{\omega \in H_{1}^{p} \mid d^{2,-1} \omega=\left(d^{2,-1}\right)^{*} \omega=0\right\}, \\
& E_{1}^{p}=\left\{\omega \in E_{0}^{p} \mid d^{1,0} \omega \in \operatorname{Image}\left(d^{2,-1}\right),\left(d^{1,0}\right)^{*} \omega \in \operatorname{Image}\left(d^{2,-1}\right)^{*}\right\} .
\end{aligned}
$$

This characterization of $E_{1}^{p}$ follows from the orthogonal decomposition

$$
\Omega^{p}=\left(\operatorname{Image} d^{2,-1}\right) \oplus\left(\operatorname{Kernel} d^{2,-1} \cap \operatorname{Kernel}\left(d^{2,-1}\right)^{*}\right) \oplus\left(\operatorname{Image}\left(d^{2,-1}\right)^{*}\right)
$$

so that if

$$
d^{1,0} \omega \in \operatorname{Image} d^{2,-1}, \quad\left(d^{1,0}\right)^{*} \omega \in \operatorname{Image}\left(d^{2,-1}\right)^{*}
$$

there is a form $\omega_{1}^{p}$ with

$$
d^{1,0} \omega+d^{2,-1} \omega_{1}=\left(d^{1,0}\right)^{*} \omega+\left(d^{2,-1}\right)^{*} \omega_{1}=0 .
$$

Thus, if we set $\omega_{\delta}=\omega+\delta \omega_{1}$ we have 


$$
d_{\delta} \omega_{\delta}, d_{\delta}^{*} \omega_{\delta} \in \delta \Omega[\delta] \Rightarrow \omega \in E_{1}^{p} .
$$

In fact, from (1.7) we see that for $\omega \in E_{0}^{p} \subset \operatorname{Kernel} d^{2,-1}$,

$$
\begin{aligned}
d^{1,0} \omega \in \operatorname{Kernel} d^{2,-1} & =\left(\operatorname{Kernel} d^{2,-1} \cap \operatorname{Kernel}\left(d^{2,-1}\right)^{*}\right) \oplus\left(\text { Image } d^{2,-1}\right) \\
& =E_{0}^{p} \oplus\left(\operatorname{Image} d^{2,-1}\right) .
\end{aligned}
$$

Therefore

$$
d^{1,0} \omega \in \text { Image } d^{2,-1} \longleftrightarrow d^{1,0} \omega \in\left(E_{0}^{p+1}\right)^{\perp} .
$$

Similarly

$$
\left(d^{1,0}\right)^{*} \omega \in \operatorname{Image}\left(d^{2,-1}\right)^{*} \longleftrightarrow\left(d^{1,0}\right)^{*} \omega \in\left(E_{0}\right)^{\perp} .
$$

Thus we can rewrite (1.7) as

$$
E_{1}^{p}=\left\{\omega \in E_{0}^{p} \mid d^{1,0} \omega,\left(d^{1,0}\right)^{*} \omega \in\left(E_{0}\right)^{\perp}\right\} .
$$

We note here that (1.5) combined with the definition of the $E_{k}^{p}$ yields

$$
\#\left\{\lambda_{i}^{p}(\delta) \in \operatorname{spectrum}\left(L_{\delta}^{p}\right) \mid \lambda_{i}^{p}(\delta) \in 0\left(\delta^{2 k}\right)\right\} \geqq \operatorname{dim} E_{k}^{p} .
$$

Remark. In [Ma-Me], Mazzeo and Melrose define spaces

$$
\bar{E}_{k}^{p}=\left\{\omega \mid \exists \omega_{1}, \ldots, \omega_{j} \text { with } L_{\delta}^{p}\left(\omega+\delta \omega_{1}+\cdots+\delta^{j} \omega_{j}\right) \in \delta^{k} \Omega^{p}[\delta]\right\} .
$$

It is clear that

So, in particular

$$
E_{k}^{p} \subseteq \bar{E}_{k-1}^{p} \subseteq E_{\left[\frac{k-1}{2}\right]}^{p}
$$

$$
E_{\infty}^{p}=\bigcap_{i=0}^{\infty} E_{i}^{p}=\bigcap_{i=0}^{\infty} \bar{E}_{i}^{p}=\bar{E}_{\infty}^{p} .
$$

We conclude this section with a proof that the sequence (1.6) stabilizes. That is, there is an $N$ such that

$$
E_{N}^{p}=E_{N+1}^{p}=\cdots=E_{\infty}^{p} .
$$

It is sufficient to prove that there is an $N^{\prime}$ with

$$
\operatorname{dim} E_{N^{\prime}}^{p}<\infty .
$$

In fact, one can take

$$
N^{\prime}=\operatorname{Min}\{\operatorname{dim} A+3, \operatorname{dim} B+3\} .
$$

Theorem 1.3. With $N^{\prime}$ as in (1.9),

$$
\operatorname{dim} E_{N^{\prime}}^{p}<\infty
$$

for every $p$.

Proof. Let

$$
\pi^{i, j}: \Omega^{*} \rightarrow \Omega^{i, j}
$$

denote the canonical projection. We will show that for every $i$,

$$
\operatorname{dim} \pi^{i, p-i} E_{N^{\prime}}^{p}<\infty .
$$


Suppose $\omega \in E_{N^{\prime}}^{p}$. Then there are $\omega_{1}, \ldots, \omega_{j}$ such that

$$
\begin{aligned}
& d_{\delta}\left(\omega+\delta \omega_{1}+\cdots+\delta^{j} \omega_{j}\right) \in \delta^{N^{\prime}} \Omega[\delta] . \\
& d_{\delta}^{*}\left(\omega+\delta \omega_{1}+\cdots+\delta^{j} \omega_{j}\right) \in \delta^{N^{\prime}} \Omega[\delta] .
\end{aligned}
$$

Write $\eta^{i, j}$ for $\pi^{i, j} \eta$. From (1.9) we see that for every $0 \leqq i \leqq p$ and $k \geqq N^{\prime}-2$,

$$
\omega^{i-k, p-i+k}=0
$$

Thus from (1.10)

$$
\begin{aligned}
& d^{2,-1} \omega^{i, p-i}=0 \\
& d^{1,0} \omega^{i, p-i}+d^{2,-1} \omega_{1}^{i-1, p-i+1}=0 \\
& \vdots \\
& d^{-1,2} \omega_{N^{\prime}-3}^{i-\left(N^{\prime}-3\right), p-i+\left(N^{\prime}-3\right)}=0 .
\end{aligned}
$$

Let

$$
\bar{\omega}=\sum_{k<i} \omega_{i-k}^{k, p-k}
$$

Then (1.12) implies

$$
d\left(\omega^{i, p-i}+\bar{\omega}\right)=0
$$

(where $d$ is the usual $d$ operator), so that

$$
\omega^{i, p-i}+\bar{\omega}=d u_{1}+h_{1}
$$

for some $u_{1} \in \Omega^{p-1}$ and $h_{1} \in \mathscr{H}^{p}(M, V)$, the (finite-dimensional) space of $g$ harmonic $p$-forms.

Similarly, let

$$
\overline{\bar{\omega}}=\sum_{k>i} \omega_{k-i}^{k, p-k}
$$

Then (1.11) implies

$$
d^{*}\left(\omega^{i, j}+\overline{\bar{\omega}}\right)=0
$$

so that

$$
\omega^{i, p-i}+\overline{\bar{\omega}}=d^{*} u_{2}+h_{2}
$$

for some $u_{2} \in \Omega^{p+1}, h_{2} \in \mathscr{H}^{p}(M, E)$. Thus

$$
\pi^{i, p-i} E_{N^{\prime}}^{p} \subset\left[\left(\bigoplus_{k<i} \Omega^{k, p-k} \oplus \Omega^{p-1} \oplus \mathscr{H}^{p}\right) \cap\left(\bigoplus_{k>i} \Omega^{k, p-k} \oplus d^{*} \Omega^{p+1} \oplus \mathscr{H}^{p}\right)\right] .
$$

Let $R^{i, p-i}$ denote the intersection on the right-hand side of (1.13). Define a linear map

$$
\tau: R^{i, p-i} \rightarrow \mathscr{H}^{p}
$$

as follows. For $\omega \in R^{l, p-l}$ we have

$$
\omega=\tilde{\omega}+d v+h
$$

for some $\tilde{\omega} \in \bigoplus_{k<i} \Omega^{k, p-k}, v \in \Omega^{p-1}, h \in \mathscr{H}^{p}$. 
Set

$$
\tau(\omega)=h .
$$

The representation (1.14) may not be unique, in which case any such representation can be chosen on a basis and $\tau$ can be extended linearly to all of $R^{i, p-i}$.

The map $\tau$ is injective. To see this, suppose

$$
\tau(\omega)=0
$$

so that $\omega \in \Omega^{i, p-i}$ satisfies

$$
\begin{aligned}
& \omega=\tilde{\omega}+d v_{1}, \\
& \omega=\tilde{\tilde{\omega}}+d^{*} v_{2}+h
\end{aligned}
$$

for $\tilde{\omega} \in \bigoplus_{k<i} \Omega^{k, p-k}, \tilde{\tilde{\omega}} \in \bigoplus_{k>i} \Omega^{k, p-k}$ and $h \in \mathscr{H}^{p}$. Then

$$
|\omega|^{2}=\langle\omega-\tilde{\omega}, \omega-\tilde{\tilde{\omega}}\rangle=\left\langle d v_{1}, d^{*} v_{2}+h_{2}\right\rangle=0
$$

so that

$$
\omega=0 \text {. }
$$

Thus

as desired.

$$
\operatorname{dim} \pi^{i, p-i} E_{N^{\prime}}^{p} \leqq \operatorname{dim} R^{i, p-i} \leqq \operatorname{dim} \mathscr{H}^{p}<\infty
$$

\section{The Sequence $\left\{E_{k}^{p}\right\}$ as a Spectral Sequence}

The goal of this section is to show that the sequence (1.6) comes equipped with the algebraic structure of a spectral sequence, and that this structure arises naturally from the Taylor series analysis of Sect. 1. Moreover, we show that the $E_{k}^{p}$ inherit a bigrading that is compatible with the differential.

We begin with some notation. In Theorem 1.3 we proved that the sequence (1.6) stabilizes. Denote by $N(p)$ the integer with the property that

$$
E_{N(p)-1}^{p} \neq E_{N(p)}^{p}=E_{\infty}^{p} .
$$

Let $\pi_{k}$ denote the orthogonal projection onto $E_{k}$, with $\pi_{k}^{\perp}=1-\pi_{k}$. Let $\widetilde{E}_{k}^{p} \subset E_{k}^{p}$ denote the orthogonal complement in $E_{k}^{p}$ of $E_{k+1}^{p}$, and $\tilde{\pi}_{k}$ the orthogonal projection onto $\widetilde{E}_{k}^{p}$ (so that $1=\sum_{i=-1}^{N-1} \tilde{\pi}_{i}+\pi_{N}$ ).

Lastly, we define $\mathscr{E}_{k}^{p} \subset \Omega[\delta]$ by

$$
\mathscr{E}_{k}^{p}=\left\{v(\delta) \in \Omega^{p}[\delta] \mid d_{\delta} v(\delta), d_{\delta}^{*} v(\delta) \in \delta^{k} \Omega[\delta]\right\}
$$

so that $v \in E_{k}^{p}$ if and only if $v$ has an extension $v(\delta) \in \mathscr{E}_{k}^{p}$, where we say $v(\delta)$ is an extension of $v$ if $v(0)=v$.

Every $v \in E_{k}$ has an extension $v_{\delta} \in \mathscr{E}_{k}$. The set of such extensions is an affine space. It will be convenient, in what follows, to have a fixed origin for this space.

For $k=0,1,2, \ldots, N-1$, let $\Phi$ be any linear extension map

$$
\Phi: \widetilde{E}_{k} \rightarrow \mathscr{E}_{k}
$$

i.e. $\Phi$ is an assignment

$$
v \in \widetilde{E}_{k} \rightarrow \Phi(v)=v+\delta v_{1}+\delta^{2} v_{2}+\cdots+\delta^{j} v_{j}
$$


such that $\Phi(v) \in \mathscr{E}_{k}$ and the map $v \rightarrow v_{i}$ is linear for every $i$.

For $k=-1$ and 0 we have

$$
\widetilde{E}_{k} \subset \mathscr{E}_{k}
$$

So we can let $\Phi$ be identity map on $\widetilde{E}_{-1}$ and $\widetilde{E}_{0}$.

Now extend $\Phi$ linearly to all of $E_{N}^{\perp}$. That is, for all $v \in E_{N}^{\perp}$,

$$
\Phi(v)=\sum_{i=0}^{N-1} \Phi\left(\tilde{\pi}_{i} v\right) .
$$

We can express any extension $v_{\delta} \in \mathscr{E}_{k}$ of $v \in E_{k}$ in terms of our fixed extension $\Phi$ as follows

Lemma 2.1. For $k \leqq N$, if $v \in \widetilde{E}_{k}$ and $v_{\delta} \in \mathscr{E}_{k}$ is any extension, then $v_{\delta}$ can be expressed uniquely as

$$
v_{\delta}=\Phi(v)+\delta r_{\delta}
$$

for some $r_{\delta} \in \mathscr{E}_{k-1}$.

Conversely, if $v_{\delta}$ is defined by (2.1) for some $r_{\delta} \in \mathscr{E}_{k-1}$, then $v_{\delta} \in \mathscr{E}_{k}$ is an extension of $v$.

Proof. If $v_{\delta} \in \mathscr{E}_{k}$ is an extension of $v$ then

$$
v_{\delta}-\Phi(v)=\delta r_{\delta}
$$

for some $r_{\delta} \in \Omega[\delta]$. Moreover, since $v_{\delta}$ and $\Phi(v) \in \mathscr{E}_{k}$ we must have

$$
\delta r_{\delta} \in \mathscr{E}_{k}
$$

which implies

$$
r_{\delta} \in \mathscr{E}_{k-1}
$$

as desired

The converse is clear.

We now define the operators that will form the basis of this section. If

$$
v_{\delta}=v_{0}+\delta v_{1}+\delta^{2} v_{2}+\cdots+\delta^{j} v_{j}
$$

is a form-valued polynomial, define the operators $\tilde{d}_{-1}, \tilde{d}_{0}, \tilde{d}_{1}, \ldots$ by

$$
d_{\delta} v_{\delta}=\delta^{-1} \tilde{d}_{-1} v_{\delta}+\tilde{d}_{0} v_{\delta}+\delta \tilde{d}_{1} v_{\delta}+\delta^{2} \tilde{d}_{2} v_{\delta}+\cdots .
$$

Similarly, define $\tilde{d}_{-1}^{*}, \tilde{d}_{0}^{*}, \tilde{d}_{1}^{*}, \ldots$

Note that $v_{\delta} \in \mathscr{E}_{k}$ if and only if

$$
\tilde{d}_{i} v_{\delta}=\tilde{d}_{\imath}^{*} v_{\delta}=0
$$

for all $i<k$.

Using the fixed extension map $\Phi$ we define linear maps

$$
d_{k}:\left(E_{N}^{p}\right)^{\perp} \rightarrow \Omega^{p+1}
$$

by

$$
d_{k} v=\tilde{d}_{k}(\Phi(v))
$$


and we similarly define their adjoints $d_{k}^{*}$. We extend these operators to all of $\Omega^{p}$ by setting for $v \in E_{N}^{p}$

$$
d_{k} v+d_{k}^{*} v+0 \text { for all } k \text {. }
$$

It is important to note that the maps $d_{k}$ and $d_{k}^{*}$ depend on our choice of the extension map $\Phi$.

The basic properties of these operators are contained in the following lemma

\section{Lemma 2.2.}

(i) For every $i>j$,

$$
\pi_{i} d_{j}=\pi_{i} d_{j}^{*}=d_{j} \pi_{i}=d_{j}^{*} \pi_{i}=0 .
$$

(ii) For every $i$ and $j$,

$$
\pi_{i} d_{i} d_{j} \pi_{j}=\pi_{i} d_{i}^{*} d_{j}^{*} \pi_{j}=0 .
$$

Proof. (i) For every $v$,

$$
\pi_{i} v=\pi_{N} v+\sum_{k=l}^{N-1} \tilde{\pi}_{k} v
$$

By definition

$$
d_{j} \pi_{N} v=d_{j}^{*} \pi_{N} v=0
$$

for all $j$. For $i \leqq k \leqq N-1, \tilde{\pi}_{k} v \in E_{k}$ so that $\Phi\left(\tilde{\pi}_{k} v\right) \in \mathscr{E}_{k}$, which implies that (since $k \geqq i>j$ )

$$
\begin{aligned}
& d_{j} \tilde{\pi}_{k} v=\tilde{d}_{j} \Phi\left(\tilde{\pi}_{k} v\right)=0, \\
& d_{j}^{*} \tilde{\pi}_{k} v=\tilde{d}_{J} \Phi\left(\tilde{\pi}_{k} v\right)=0 .
\end{aligned}
$$

Adding (2.2) and (2.3) yields

$$
d_{j} \pi_{l}=d_{j}^{*} \pi_{i}=0 \quad \text { for } i>j .
$$

Taking adjoints yields

$$
\pi_{i} d_{j}=\pi_{l} d_{j}^{*}=0 \quad \text { for } i>j
$$

which proves (i).

(ii) From (i) we have

$$
\pi_{i} d_{i} d_{j} \pi_{j}=\tilde{\pi}_{i} d_{i} d_{j} \tilde{\pi}_{j}
$$

For any $v \in \Omega^{p}, \omega \in \Omega^{p+2}$ we have, since $d_{\delta}^{2}=0$

$$
0=\left\langle d_{\delta} \Phi\left(\tilde{\pi}_{i} v\right), d_{\delta}^{*} \Phi\left(\tilde{\pi}_{j} w\right)\right\rangle=\delta^{i+j}\left\langle\tilde{d}_{i} \Phi\left(\tilde{\pi}_{i} v\right), \tilde{d}_{j}^{*} \Phi\left(\tilde{\pi}_{j} w\right)\right\rangle+0\left(\delta^{l+j+1}\right) .
$$

Thus

$$
0=\left\langle\tilde{d}_{i} \Phi\left(\tilde{\pi}_{i} v\right), \tilde{d}_{j}^{*} \Phi\left(\tilde{\pi}_{j} w\right)\right\rangle=\left\langle d_{i} \tilde{\pi}_{i} v, d_{j}^{*} \pi_{j} w\right\rangle=\left\langle\tilde{\pi}_{j} d_{j} d_{i} \tilde{\pi}_{i} v, w\right\rangle,
$$

which implies

$$
\pi_{j} d_{j} d_{i} \pi_{i}=\tilde{\pi}_{j} d_{j} d_{i} \tilde{\pi}_{i}=0
$$

Taking adjoints yields

$$
\pi_{j} d_{j}^{*} d_{l}^{*} \pi_{l}=\tilde{\pi}_{j} d_{j}^{*} d_{l}^{*} \tilde{\pi}_{l}=0
$$


Lemma 2.2, part (i) has the following important corollary:

Lemma 2.3. The maps $\pi_{k} d_{k} \pi_{k}$ and $\pi_{k} d_{k}^{*} \pi_{k}$ are independent of our choice of the extension map $\Phi$.

Proof. From Lemma 2.2 (i), $\pi_{k} d_{k} \pi_{k}=\tilde{\pi} d_{k} \tilde{\pi}_{k}$. For $v \in \Omega^{p}, \tilde{\pi}_{k} d_{k} \tilde{\pi}_{k} v=\tilde{\pi}_{k} \tilde{d}_{k} \Phi\left(\tilde{\pi}_{k} v\right)$. Suppose $\Phi^{\prime}$ is any other extension map, then

$$
\left.\Phi\left(\tilde{\pi}_{k} v\right)-\Phi^{\prime}\left(\tilde{\pi}_{k} v\right)=\delta \omega\right) \delta
$$

for some $\omega_{\delta} \in \mathscr{E}_{k-1}$ (Lemma 2.1). Thus

$$
\tilde{\pi}_{k} \tilde{d}_{k}\left(\Phi\left(\tilde{\pi}_{k} v\right)-\Phi^{\prime}\left(\tilde{\pi}_{k} v\right)\right)=\tilde{\pi}_{k} d_{k-1} \omega_{\delta}=0
$$

by Lemma 2.2 (i). Therefore

$$
\pi_{k} \tilde{d}_{k} \Phi\left(\tilde{\pi}_{k} v\right)=\pi_{k} \tilde{d}_{k} \Phi^{\prime}\left(\tilde{\pi}_{k} v\right)
$$

and this implies $\pi_{k} d_{k} \pi_{k}$ is independent of $\Phi$. The same proof holds for $\pi_{k} d_{k}^{*} \pi_{k}$.

Our next goal is to show that for every $v \in E_{k}$ there is an extension $v_{\delta}^{*}$ such that

$$
\tilde{d}_{k} v_{\delta}^{*} \in \tilde{E}_{k}, \quad \tilde{d}_{k}^{*} v_{\delta}^{*} \in \tilde{E}_{k},
$$

so that

$$
\begin{aligned}
& \tilde{d}_{k} v_{\delta}^{*}=\left(\pi_{k} d_{k} \pi_{k}\right) v, \\
& \tilde{d}_{k}^{*} v_{\delta}^{*}=\left(\pi_{k} d_{k}^{*} \pi_{k}\right) v .
\end{aligned}
$$

It is from this fact that we derive all of our desired results.

Our proof of this fact begins with the definition of two more operators:

$$
\begin{aligned}
& D_{k}: E_{k+1}^{\perp} \rightarrow E_{k+1}^{\perp}, \\
& \bar{D}_{k}: E_{k+1}^{\perp} \rightarrow E_{k+1}^{\perp},
\end{aligned}
$$

where we set

$$
\begin{aligned}
& D_{k}=\sum_{i=-1}^{k} \tilde{\pi}_{i} d_{i}, \\
& \bar{D}_{k}=\sum_{i=-1}^{k} d_{i} \tilde{\pi}_{i} .
\end{aligned}
$$

These operators depend on the extension $\Phi$. Note that Lemma 2.2 (ii) implies

$$
D_{k} \bar{D}_{k}=\bar{D}_{k}^{*} D_{k}^{*}=0
$$

for all $k$. Now we define the "Laplacian" $\square_{k}$ by

$$
\square_{k}=D_{k} D_{k}^{*}+\bar{D}_{k}^{*} \bar{D}_{k}=\left(D_{k}+\bar{D}_{k}^{*}\right)\left(D_{k}^{*}+\bar{D}_{k}\right): E_{k+1}^{\perp} \rightarrow E_{k+1}^{\perp} .
$$

\section{Theorem 2.4.}

(a) Fix $v \in E_{k}^{p}$, then

(i) There is an extension $v_{\delta}^{*} \in \mathscr{E}$ of $v$ with

$$
\tilde{d}_{k} v_{\delta}^{*} \in E_{k}^{p+1}, \quad \tilde{d}_{k}^{*} v_{\delta}^{*} \in E_{k}^{p-1} .
$$


(ii) The polynomial $v_{\delta}^{*}$ from part (i) is unique modulo $\delta \mathscr{E}_{k}^{p}$.

(iii) In terms of the extension $\Phi, v_{\delta}^{*}=\Phi(v)+\sum_{i=-1}^{k-1} \delta^{k-i} \Phi\left(v_{i}\right)$, where

$$
v_{i}=\tilde{\pi}_{i} \square_{k-1}^{-1}\left(\bar{D}_{k-1}^{*} d_{k}+D_{k-1} d_{k}^{*}\right) v .
$$

(b) For every $p$ and $k$, the operator

$$
\square_{k}^{p}:\left(E_{k+1}^{p}\right)^{\perp} \rightarrow\left(E_{k+1}^{p}\right)^{\perp}
$$

is invertible.

Proof. We will prove (a) and (b) simultaneously inductively on $k$.

$k=-1$ : Part (a) is trivial since for every $v \in E_{-1}^{p}=\Omega^{p}$ and every extension $v_{\delta}$ of $v$ we have

$$
\begin{aligned}
& \tilde{d}_{-1} v_{\delta}=d^{2,-1} v \in \tilde{E}_{-1}, \\
& \tilde{d}_{-1}^{*} v_{\delta}=\left(d^{2,-1}\right)^{*} v \in \tilde{E}_{-1} .
\end{aligned}
$$

Thus any extension will satisfy part (i). Parts (ii) and (iii) follow trivially since every two extensions of $v$ differ by an element of $\delta \mathscr{E}_{0}=\delta \Omega[\delta]$.

Part (b) follows from the observation

$$
\begin{aligned}
\operatorname{Kernel} \square_{-1} & =\operatorname{Kernel}\left(\left(d^{2,-1}\right)^{*} d^{2,-1}+d^{2,-1}\left(d^{2-1}\right)^{*}\right) \\
& =\operatorname{Kernel}\left(d^{2,-1}\right)^{*} \cap \operatorname{Kernel}\left(d^{2,-1}\right)=E_{0} .
\end{aligned}
$$

Now assume (a) and (b) are true for $k-1$.

Proof of $(a)$. Given $v \in E_{k}^{p}$ define an extension $v_{\delta}^{*}$ by the formula in part (iii), i.e.

$$
v_{\delta}^{*}=\Phi(v)+\sum_{i=-1}^{k-1} \delta^{k-i} \Phi\left(v_{i}\right)
$$

where

$$
v_{i}=\tilde{\pi}_{i} \square_{k-1}^{-1}\left(\bar{D}_{k-1}^{*} d_{k}+D_{k-1} d_{k}^{*}\right) v
$$

Note that for $v \in E_{k}$,

$$
d_{k} v=d_{k} \tilde{\pi}_{k} v, \quad d_{k}^{*} v=d_{k}^{*} \tilde{\pi}_{k} v
$$

(we have used Lemma 2.2 (i)). It follows from Lemma 2.2 (ii) that

$$
\begin{aligned}
& \bar{D}_{k-1}^{*} d_{k}^{*} v=\bar{D}_{k-1}^{*} d_{k}^{*} \tilde{\pi}_{k} v=0, \\
& D_{k-1} d_{k} v=D_{k-1} d_{k} \tilde{\pi}_{k} v=0 .
\end{aligned}
$$

Thus (2.4) can be written

$$
v_{l}=\tilde{\pi}_{i} \square_{k-1}^{-1}\left(\bar{D}_{k-1}^{*}+D_{k-1}\right)\left(d_{k}+d_{k}^{*}\right) v .
$$

It is easy to check that $\omega_{\delta}$ is an extension of $v$. Moreover

$$
\begin{aligned}
\tilde{d}_{k} v_{\delta}^{*} & =\tilde{d}_{k} \Phi(v)+\sum_{i=-1}^{k-1} \tilde{d}_{i} \Phi\left(v_{i}\right)=d_{k} v+\sum_{i=-1}^{k-1} d_{i} v_{l} \\
& =d_{k} v+\left(\sum_{i=-1}^{k-1} d_{i} \tilde{\pi}_{i}\right) \square_{k-1}^{-1}\left(\bar{D}_{k-1}^{*}+D_{k-1}\right)\left(d_{k}+d_{k}^{*}\right) v \\
& =d_{k} v+\overline{D_{k-1}} \square_{k-1}^{-1}\left(\bar{D}_{k-1}^{*}+D_{k-1}\right)\left(d_{k}+d_{k}\right)^{*} v .
\end{aligned}
$$


Similarly

$$
\tilde{d}_{k}^{*} v_{\delta}^{*}=d_{k}^{*} v+D_{k-1}^{*} \square_{k-1}^{-1}\left(\bar{D}_{k-1}^{*}+D_{k-1}\right)\left(d_{k}+d_{k}^{*}\right) v
$$

Adding (2.5) and $* 2.6)$ yields

$$
\left(\tilde{d}_{k}+\tilde{d}_{k}^{*}\right) v_{\delta}^{*}=\left(d_{k}+d_{k}^{*}\right) v+\left(\bar{D}_{k-1}+D_{k-1}^{*}\right) \square_{k-1}^{-1}\left(\bar{D}_{k-1}^{*}+D_{k-1}\right)\left(d_{k}+d_{k}^{*}\right) v .
$$

Writing $\square_{k-1}=\left(\bar{D}_{k-1}^{*}+D_{k-1}\right)\left(\bar{D}_{k-1}+D_{k-1}^{*}\right)$ we see that

$$
\begin{aligned}
& \left(\bar{D}_{k-1}+D_{k-1}^{*}\right)\left(\square_{k-1}\right)^{-1}\left(\bar{D}_{k-1}^{*}+D_{k-1}\right) \\
& \left.\quad=\text { orthogonal projection into (Kernel } \square_{k-1}\right)^{\perp} \\
& \quad=\text { orthogonal projection onto } E_{k}^{\perp}
\end{aligned}
$$

(the inductive hypotheses implies Kernel $\square_{k-1}=E_{k}$ ). It follows from Lemma 2.2 (i) that

$$
\left(d_{k}+d_{k}^{*}\right) v \in E_{k+1}^{\perp} .
$$

Thus (2.7) says

$$
\left(\tilde{d}_{k}+\tilde{d}_{k}^{*}\right) v_{\delta}^{*}=\tilde{\pi}_{k}\left(d_{k}+d_{k}^{*}\right) v=\tilde{\pi}_{k} d_{k} v+\tilde{\pi}_{k} d_{k}^{*} v
$$

Now

$$
\begin{aligned}
& \tilde{d}_{k} v_{\delta}^{*}, \tilde{\pi}_{k} d_{k} v \in \Omega^{p+1}, \\
& \tilde{d}_{k}^{*} v_{\delta}^{*}, \tilde{\pi}_{k} d_{k}^{*} v \in \Omega^{p-1} .
\end{aligned}
$$

Therefore, (2.8) implies

$$
\begin{aligned}
& \tilde{d}_{k} v_{\delta}^{*}=\tilde{\pi}_{k} d_{k} v \in \tilde{E}_{k} \subset E_{k}, \\
& \tilde{d}_{k}^{*} v_{\delta}^{*}=\tilde{\pi}_{k} d_{k}^{*} v \in \tilde{E}_{k} \subset E_{k} .
\end{aligned}
$$

Thus $v_{\delta}^{*}$ as defined in part (iii) satisfies the conclusion of part (i).

If $v_{\delta}$ and $v_{\delta}^{\prime}$ are any two extensions of $v$ in $\mathscr{E}_{k}$, then

$$
v_{\delta}-v_{\delta}^{\prime}=\delta r_{\delta}
$$

for some $r_{\delta} \in \mathscr{E}_{k-1}$.

If

$$
\tilde{d}_{k} v_{\delta}=\tilde{d}_{k} v_{\delta}^{\prime}, \quad \tilde{d}_{k}^{*} v_{\delta}=\tilde{d}_{k}^{*} v_{\delta}^{\prime}
$$

then

$$
0=\tilde{d}_{k}\left(\delta r_{\delta}\right)=\tilde{d}_{k-1} r_{\delta}
$$

Similarly,

$$
0=\tilde{d}_{k-1}^{*} r_{\delta} .
$$

Thus $r_{\delta} \in \mathscr{E}_{k}$. This shows that $v_{\delta}^{*}$ of part (i) is unique modulo $\delta \mathscr{E}_{k}$, which is part (ii).

Proof of $(b)$. Note that

$$
\begin{aligned}
& D_{k}=D_{k-1}+\tilde{\pi}_{k} \tilde{d}_{k}, \\
& \bar{D}_{k}=\overline{D_{k-1}}+\tilde{d}_{k} \tilde{\pi}_{k} .
\end{aligned}
$$


Using the decomposition

$$
E_{k}^{\perp}=E_{k-1}^{\perp} \oplus \tilde{E}_{k}
$$

we express the operator $\square_{k}$ as a $2 \times 2$ matrix of operators

$$
\left(\begin{array}{cc} 
& D_{k-1}^{*} d_{k}+ \\
\square_{k-1} & \bar{D}_{k-1}^{*} d_{k}^{*} \\
d_{k} D_{k-1}+ & d_{k} d_{k}^{*}+ \\
d_{k}^{*} \bar{D}_{k-1} & d_{k}^{*} d_{k}
\end{array}\right) .
$$

For simplicity, relabel this matrix

$$
\left(\begin{array}{ll}
A_{k} & B_{k} \\
B_{k}^{*} & C_{k}
\end{array}\right)
$$

By induction, $A_{k}$ is invertible.

$C_{k}$ is also invertible, since if $v \in E_{k}$ and $C_{k} v=0$ then

$$
\left\langle C_{k} v, v\right\rangle=0
$$

from which it follows that

$$
d_{k} v=\tilde{d}_{k} \Phi(v)=0, \quad d_{k}^{*} v=\tilde{d}_{k}^{*} \Phi(u)=0 .
$$

Thus $\Phi(v) \in \mathscr{E}_{k+1}$ which implies

$$
v \in E_{k+1} \subset E_{k}^{\perp}
$$

so $v=0$.

Formally, the inverse of $\square_{k}$ is given by the $2 \times 2$ matirx of operators

$$
\left(\begin{array}{cc}
\left(A_{k}-B_{k} C_{k}^{-1} B_{k}^{*}\right)^{-1} & -A_{k}^{-1} B_{k}\left(C_{k}-B_{k}^{*} A_{k}^{-1} B_{k}\right)^{-1} \\
-C_{k}^{-1} B_{k}^{*}\left(A_{k}-B_{k} C_{-1}+k B_{k}^{*}\right)^{-1} & \left(C_{k}-B_{k}^{*} A_{k}^{-1} B_{k}\right)^{-1}
\end{array}\right) .
$$

The invertibility of $\square_{k}$ follows once we know

$$
A_{k}-B_{k} C_{k}^{-1} B_{k}^{*}
$$

and

$$
C_{k}-B_{k}^{*} A_{k}^{-1} B_{k}
$$

are invertible.

For $v \in E_{k}$

$$
\begin{aligned}
& \left(C_{k}-B_{k}^{*} A_{k}^{-1} B_{k}\right) v \\
& \quad=\left(\tilde{\pi}_{k} d_{k}+\tilde{\pi}_{k} d_{k}^{*}\right)\left[1-\left(\bar{D}_{k-1}+D_{k-1}^{*}\right) \square_{k-1}^{-1}\left(\bar{D}_{k-1}^{*}+D_{k-1}\right)\right]\left(d_{k} \tilde{\pi}_{k}+d_{k}^{*} \tilde{\pi}_{k}\right) v .
\end{aligned}
$$

But

$$
\begin{aligned}
1- & \left(\bar{D}_{k-1}+D_{k-1}^{*}\right) \square_{k-1}^{-1}+D_{k-1} \\
& \left.=\text { orthogonal projection onto }\left(\text { Kernel } \square_{k-1}\right)^{\perp}=E_{k}\right)
\end{aligned}
$$

(by induction). Since

$$
\text { Image } d_{k} \text {, Image } d_{k}^{*} \subset E_{k+1}^{\perp}
$$


(from Lemma 2.2 (i)) (2.9) equals

$$
\left(\tilde{\pi}_{k} d_{k}+\tilde{\pi}_{k} d_{k}^{*}\right) \tilde{\pi}_{k}\left(d_{k} \tilde{\pi}_{k}+d_{k}^{*} \tilde{\pi}_{k}\right) v .
$$

If $\left(C_{k}-B_{k}^{*} A_{k-1}^{-1} B_{k}\right) v=0$ then

$0=\left\langle\left(C_{k}-B_{k}^{*} A_{k-1}^{-1} B_{k}\right) v, v\right\rangle=\left|\tilde{\pi}_{k}\left(d_{k}+d_{k}^{*}\right) \tilde{\pi}_{k} v\right|^{2} \Rightarrow\left(\tilde{\pi}_{k} d_{k} \tilde{\pi}_{k}+\tilde{\pi}_{k} d_{k}^{*} \tilde{\pi}_{k}\right) v=0$.

Since $\tilde{\pi}_{k} d_{k} \tilde{\pi}_{k} v \in \Omega^{p+1}, \tilde{\pi}_{k} d_{k}^{*} \tilde{\pi}_{k} v \in \Omega^{p-1}(2.10)$ implies

$$
\begin{aligned}
& 0=\tilde{\pi}_{k} d_{k} \tilde{\pi}_{k} v=\tilde{d}_{k} v_{\delta}^{*}, \\
& 0=\tilde{\pi}_{k} d_{k}^{*} \tilde{\pi}_{k} v=\tilde{d}_{k}^{*} v_{\delta}^{*} .
\end{aligned}
$$

Thus $v_{\delta}^{*} \in \mathscr{E}_{k+1} \Rightarrow v \in E_{k+1} \subset E_{k}^{\perp}$ so $v=0$. Therefore $C_{k}-B_{k}^{*} A_{k} B_{k}$ in invertible.

Now we will see that $A_{k}-B_{k} C_{k}^{-1} B_{k}^{*}$ is invertible. Suppose $v \in E_{k}^{\perp}$ and let $z=-C_{k}^{-1} B_{k}^{*} v \in \tilde{E}_{k}$. Define an extension $z_{\delta}$ of $z$ by

$$
z_{\delta}=\Phi(z)+\sum_{i=-1}^{k-1} \delta^{k-1} \Phi\left(\tilde{\pi}_{i} v\right)
$$

Then

$$
\tilde{d}_{k} z_{\delta}=\tilde{d}_{k} \Phi(z)+\sum_{i=-1}^{k-1} \tilde{d}_{i} \Phi\left(\tilde{\pi}_{l} v\right)=d_{k} z+\sum_{i=-1}^{k-1} d_{i} \tilde{\pi}_{l} v=d_{k} z+\bar{D}_{k-1} v
$$

Similarly

$$
\tilde{d}_{k}^{*} z_{\delta}=d_{k}^{*} z+d_{k-1}^{*} v
$$

So we have

$$
\begin{aligned}
\left|\tilde{d}_{k} z_{\delta}\right|^{2}+\left|\tilde{d}_{k}^{*} z_{\delta}\right|^{2} \\
=\left|d_{k} z+\bar{D}_{k-1} v\right|^{2}+\left|d_{k}^{*} z+d_{k-1}^{*} v\right|^{2} \\
=\left\langle\bar{D}_{k-1}^{*} d_{k} z+\bar{D}_{k-1}^{*} \bar{D}_{k-1} v+D_{k-1} d_{k}^{*} z+D_{k-1} D_{k-1}^{*} v, v\right\rangle \\
\quad+\left\langle d_{k}^{*} d_{k} z+d_{k}^{*} \bar{D}_{k-1} v+d_{k} d_{k}^{*} z+d_{k} D_{k-1}^{*} v, z\right\rangle \\
=\left\langle B_{k} z+A_{k} v, v\right\rangle+\left\langle C_{k} z+B_{k}^{*} v, z\right\rangle .
\end{aligned}
$$

Now use $z=-C_{k}^{-1} B_{k}^{*} v$ to find

$$
\begin{aligned}
(2.11) & =\left\langle\left(A_{k}-B_{k} C_{k}^{-1} B_{k}^{*}\right) v, v\right\rangle+\left\langle\left(-C_{k}\left(C_{k}^{-1} B_{k}^{*}\right)+B_{k}^{*}\right) v, z\right\rangle \\
& =\left\langle\left(A_{k}-B_{k} C_{k}^{-1} B_{k}^{*}\right) v, v\right\rangle .
\end{aligned}
$$

Therefore, if

$$
\left(A_{k}-B_{k} C_{k}^{-1} b_{k}^{*}\right) v=0
$$

then

$$
\left|\tilde{d}_{k} z_{\delta}\right|^{2}+\left|\tilde{d}_{k}^{*} z_{\delta}\right|^{2}=0 \Rightarrow \tilde{d}_{k} z_{\delta}=\tilde{d}_{k}^{*} z_{\delta}=0 .
$$

But this implies

$$
z_{\delta} \in \mathscr{E}_{k+1} \Rightarrow z \in E_{k+1} \subset E_{k}^{\perp}
$$


Thus, we must have

$$
0=z=-C_{k}^{-1} B_{k}^{*} v
$$

But this implies

$$
\left(A_{k}-B_{k} C_{k}^{-1} B_{k}^{*}\right) v=A_{k} v=0 .
$$

This contradicts the invertibility of $A_{k}$. Therefore $A_{k}-B_{k} C_{k}^{-1} B_{k}^{*}$ is invertible.

In what follows, a fundamental role will be played by the operator

$$
\pi_{k} d_{k} \pi_{k}: E_{k}^{p} \rightarrow E_{k}^{p+1}
$$

(which, by Lemma 2.3, is well-defined independent of our choice of the extension $\Phi)$ and the associated Laplacian

$$
\Delta_{k}=\pi_{k} d_{k} \pi_{k} d_{k}^{*} \pi_{k}+\pi_{k} d_{k}^{*} \pi_{k} d_{k}^{*} \pi_{k}: E_{k}^{p} \rightarrow E_{k}^{p} .
$$

The significance of these operators follows from:

\section{Theorem 2.5.}

a) $\left(\pi_{k} d_{k} \pi_{k}\right)^{2}=\left(\pi_{k} d_{k}^{*} \pi_{k}\right)^{2}=0$.

b) The kernel of the operator

$$
\Delta_{k}: E_{k}^{p} \rightarrow E_{k}^{p}
$$

is precisely $E_{k+1}^{p}$.

Before we prove this theorem, we introduce a very useful definition. Up to this point, we have defined all operators with respect to an arbitrary extension map $\Phi$. Theorem 2.4 provides us with a canonical choice for $\Phi$.

Definition. Define, for $v \in \tilde{E}_{k}$. The extension map $\Phi^{\prime}$ by

$$
\Phi^{\prime}(v)=v_{\delta}^{*},
$$

where $v_{\delta}^{*}$ is defined by the formula given in Theorem 2.4 (a) (iii). We let $d_{k}^{\prime}$ denote the operator $d_{k}$ associated to the extension $\Phi^{\prime}$. That is, for $v \in \tilde{E}_{k}$,

$$
d_{k}^{\prime} v=\tilde{d}_{k} v_{\delta}^{*} .
$$

From Theorem 2.4 (a) (i) we know that for $v \in E_{k}$,

$$
d_{k}^{\prime} v,\left(d_{k}^{\prime}\right)^{*} v \in E_{k} .
$$

This choice of extension greatly simplifies the proofs in this and the next section.

Proof of Theorem 2.5. a) Since, by Lemma 2.3, $\pi_{k} d_{k} \pi_{k}$ is independent of our extension so we can choose the extension $\Phi^{\prime}$ defined above. This gives

$$
\pi_{k} d_{k} \pi_{k} d_{k} \pi_{k} v=\pi_{k} d_{k}^{\prime} \pi_{k} d_{k}^{\prime} \pi_{k} v
$$

But the image of $d_{k}^{\prime} \pi_{k}$ is contained in $E_{k}$ so that

$$
\pi_{k} d_{k}^{\prime} \pi_{k}=d_{k}^{\prime} \pi_{k} .
$$

Thus

$$
\pi_{k} d_{k} \pi d_{k} \pi_{k}=\pi_{k} d_{k}^{\prime} d_{k}^{\prime} \pi_{k} v=0
$$


The last equality follows from Lemma 2.2 (ii) which states that $\pi_{k} d_{k} d_{k} \pi_{k}=0$, where $d_{k}$ is defined relative to any extension $\Phi$. Applying this lemma to the extension $\Phi^{\prime}$ yields the desired equality.

b) Clearly $E_{k+1} \subset$ Kernel $\Delta_{k}$.

To prove the converse note that for $v \in E_{k}, v \in \operatorname{Kernel} \Delta_{k}$ implies

$$
\left\langle\Delta_{k} v, v\right\rangle=0
$$

from which it follows that

$$
\begin{aligned}
& \pi_{k} d_{k} \pi_{k} v=\pi_{k} d_{k} v=0, \\
& \pi_{k} d_{k}^{*} \pi_{k} v=\pi_{k} d_{k}^{*} v=0 .
\end{aligned}
$$

Thus

$$
\begin{aligned}
& \tilde{d}_{k} \Phi^{\prime}(v)=\pi_{k} d_{k} v=0, \\
& \tilde{d}_{k}^{*} \Phi^{\prime}(v)=\pi_{k} d_{k}^{*} v=0 .
\end{aligned}
$$

Therefore $\Phi^{\prime}(v) \in \mathscr{E}_{k+1}$ which implies $v \in E_{k+1}$ as desired.

In addition to providing the fundamental properties of the operators $\tilde{\pi}_{k} d_{k}^{\prime} \tilde{\pi}_{k}$ and $\Delta_{k}$, Theorem 2.5 implies the following important observation concerning the higher order terms

$$
d_{k+j}^{\prime} v \equiv \tilde{d}_{k+j} \Phi^{\prime}(v)
$$

for $v \in E_{k}$ and $j>0$.

Recall that $\Phi^{\prime}$ was defined so that

$$
\begin{aligned}
d_{k}^{\prime} v & \equiv \tilde{d}_{k} \Phi^{\prime}(v) \in E_{k}, \\
\left(d_{k}^{\prime}\right)^{*} v & \equiv \tilde{d}_{k}^{*} \Phi^{\prime}(v) \in E_{k} .
\end{aligned}
$$

In fact we have:

Corollary 2.6. If $v \in E_{k}^{p}$ then there is a polynomial

$$
v_{\delta}=v+\delta v_{1}+\ldots+\delta^{l} v_{i}
$$

such that

$$
\begin{gathered}
\tilde{d}_{j} v_{\delta}=\tilde{d}_{j}^{*} v_{\delta}=0 \quad \text { for } j<k, \\
\tilde{d}_{k} v_{\delta}, \tilde{d}_{k}^{*} v_{\delta} \in E_{k}, \\
\tilde{d}_{k+j} v_{\delta}, \tilde{d}_{k+j}^{*} v_{\delta} \in E_{k+j-1} \quad \text { for } j>0 .
\end{gathered}
$$

Proof. The extension $\Phi^{\prime}$ defined earlier satisfies (2.12) and (2.13). We will modify $\Phi^{\prime}$ to satisfy (2.14).

For any $l$ and any $w \in \widetilde{E}_{l}$,

$$
\begin{aligned}
0= & \left\langle d_{\delta} \Phi^{\prime}(v), d_{\delta}^{*} \Phi^{\prime}(w)\right\rangle \\
= & \delta^{k+l}\left\langle d_{k}^{\prime} v,\left(d_{l}^{\prime}\right)^{*} w\right\rangle \\
& +\delta^{k+l+1}\left\langle d_{k}^{\prime} v,\left(d_{l+1}^{\prime}\right)^{*} w\right\rangle+\left\langle d_{k+l}^{\prime} v,\left(d_{l}^{\prime}\right)^{*} w\right\rangle \\
& +0\left(\delta^{k+l+2}\right) .
\end{aligned}
$$


In particular

$$
\begin{aligned}
0 & =\left\langle d_{k}^{\prime} v,\left(d_{l+1}^{\prime}\right)^{*} w\right\rangle+\left\langle d_{k+l}^{\prime} v,\left(d_{l}^{\prime}\right)^{*} w\right\rangle \\
& =\left\langle\left(\tilde{\pi}_{l} d_{l+1}^{\prime} d_{k}^{\prime}+\tilde{\pi}_{l} d_{l}^{\prime} \tilde{\pi}_{l} d_{k+l}^{\prime}\right) v, w\right\rangle .
\end{aligned}
$$

Suppose $l+1 \leqq k$. Then $\tilde{\pi}_{l} d_{l+l}^{\prime} d_{k}^{\prime}=0$. This can be seen as follows: If $l+1<k$, then since $d_{k}^{\prime} v \in E_{k}$ we have $d_{l+l}^{\prime} d_{k}^{\prime} v=0$ (Lemma 2.2 (i)).

If $l+1=k$, then

$$
\tilde{\pi}_{l} d_{l+l}^{\prime} d_{k}^{\prime} v=\tilde{\pi}_{k-1} d_{k}^{\prime} d_{k}^{\prime} v
$$

We have

$$
v \in E_{k} \Rightarrow d_{k}^{\prime} v \in E_{k} \Rightarrow d_{k}^{\prime} d_{k}^{\prime} v \in E_{k} \Rightarrow \tilde{\pi}_{k-1} d_{k}^{\prime} d_{k}^{\prime} v=0 .
$$

Thus, from (2.16), for all $l \leqq k-1$ and $w \in \widetilde{E}_{l}$,

$$
\left\langle\tilde{\pi}_{l} d_{l}^{\prime} \tilde{\pi}_{l} d_{k+1}^{\prime} v, w\right\rangle=0,
$$

which implies for all $l \leqq k-1$,

$$
d_{k+1}^{\prime} v \in \operatorname{Kernel} \tilde{\pi}_{l} d_{l}^{\prime} \tilde{\pi}_{l} .
$$

From Theorem 2.5(b), the sequence

$$
\tilde{\pi}_{l}\left(d_{l}^{\prime}\right)^{*} \tilde{\pi}_{l}: \widetilde{E}_{l}^{n} \rightarrow \widetilde{E}_{l}^{n-1} \rightarrow \widetilde{E}_{l}^{n-2} \rightarrow \cdots
$$

is exact. That is,

$$
\text { Kernel }\left.\tilde{\pi}_{l} d_{l}^{\prime} \tilde{\pi}_{l}\right|_{\widetilde{E}_{l}}=\text { Image } \tilde{\pi}_{l} d_{l}^{\prime} \tilde{\pi}_{l} .
$$

So that, for $l \leqq k-1$,

$$
\tilde{\pi}_{l} d_{k+1}^{\prime} v \in \text { Image } \tilde{\pi}_{l} d_{l}^{\prime} \tilde{\pi}_{l}
$$

Similarly, for $l \leqq k-1$,

$$
\tilde{\pi}_{l}\left(d_{k+1}^{\prime}\right)^{*} v \in \text { Image } \tilde{\pi}_{l}\left(d_{l}^{\prime}\right)^{*} \tilde{\pi}_{l} .
$$

Thus, for every $l \leqq k-1$ we can find a $u_{l} \in E_{l}$ such that

$$
\begin{aligned}
d_{l}^{\prime} u_{l} & =\tilde{\pi}_{l} d_{k+1} v, \\
\left(d_{l}^{\prime}\right)^{*} u_{l} & =\tilde{\pi}_{l} d_{k+1}^{*} v .
\end{aligned}
$$

Let

$$
\Phi_{1}^{\prime}(v)=\Phi^{\prime}(v)-\sum_{i=-1}^{k-1} \delta^{k-l+1} \Phi\left(u_{l}\right)
$$

Then $\Phi_{1}^{\prime}$ satisfies (2.12), (2.13) and (2.14) for $j=1$. Continuing inductively, setting the coefficient of $\delta^{k+1+j}$ in (2.15) equal to 0 yields the desired extension.

Remark. Theorem 2.5(a) implies that for each $k \geqq-1$ we have a complex

$$
0 \longrightarrow E_{k}^{0 \pi_{k} d_{k} \pi_{k}} E_{k}^{1} \stackrel{\pi_{k} d_{k} \pi_{k}}{\longrightarrow} E_{k}^{2} \longrightarrow \cdots \text {. }
$$

Theorem 2.5(b) implies that the cohomology of this sequence at the $i^{\text {th }}$ step is isomorphic to $E_{k+1}^{i}$. 
Thus the sequence of complexes has the structure of a spectral sequence. In Sect. 3 of this paper we prove that this spectral sequence converges to the cohomology of $M$. In the case that $M$ is a fiber bundle, and the vector bundle is trivial, this is Theorem 7 of [Ma-Me]. Dai has observed ([Dai]) that in this case the above analytic sequence is isomorphic to the Leray spectral sequence. We also prove this in Sect. 4 as a special case of a more general theorem.

Before leaving this section, we prove that the spectral sequence comes equipped with more structure, namely a bigrading inherited from the bigrading on $\Omega^{*}(M, V)$. Let

$$
E_{k}^{a, b}=\pi^{a, b} E_{k},
$$

where

$$
\pi^{a, b}: \Omega^{*}(M, V) \rightarrow \Omega^{a, b}(M, V)
$$

denotes the canonical projection. We will prove that

$$
E_{k}^{p}=\bigoplus_{a+b=p} E_{k}^{a, b}
$$

and that

$$
\begin{aligned}
& \pi_{k} d_{k} \pi_{k}\left(E_{k}^{a, b}\right) \subseteq E_{k}^{a-k+1, b+k}, \\
& \pi_{k} d_{k}^{*} \pi_{k}\left(E_{k}^{a, b}\right) \subseteq E_{k}^{a+k-1, b-k} .
\end{aligned}
$$

We begin with a lemma which will also play a crucial role in Sect. 4 when we prove the equivalence between our spectral sequence and the Leray spectral sequence.

Let

$$
\mathscr{D}_{k}=\left\{\omega_{\delta} \in \Omega[\delta] \mid d_{\delta} \omega_{\delta} \in \delta^{k} \Omega[\delta]\right\}
$$

We define a map

$$
\tilde{d}_{k}: \mathscr{D}_{k} \rightarrow \Omega
$$

by

$$
\tilde{d}_{k} \omega_{\delta}=\lim _{\delta \rightarrow 0} \delta^{-k} d_{\delta} \omega_{\delta}
$$

Furthermore, let

$$
D_{k}=\left\{\omega \in \Omega \mid \exists \omega_{\delta} \in \mathscr{D}_{k} \text { with } \omega_{\delta}(0)=\omega\right\}
$$

Then we have

Lemma 2.7. For all $k$

$$
D_{k}=\bigoplus_{l=-1}^{k-1} \operatorname{Image} \pi_{i} d_{i} \pi_{i}+E_{k}
$$

$$
\tilde{d}_{k} \mathscr{D}_{k}=\bigoplus_{i=-1}^{k} \text { Image } \pi_{i} d_{i} \pi_{i}
$$

Proof. Proof of (i): We have

$$
E_{-1}=\Omega^{*}=\text { Image } \pi_{-1} d_{-1} \pi_{-1}+\text { Image } \pi_{-1} d_{-1}^{*} \pi_{-1}+E_{0} .
$$


Similarly,

$$
E_{0}=\operatorname{Image} \pi_{0} d_{0} \pi_{0}+\operatorname{Image} \pi_{0} d_{0}^{*} \pi_{0}+E_{1} .
$$

Continuing in this fashion, for every $k$

$$
\Omega^{*}=\bigoplus_{i=-1}^{k-1} \text { Image } \pi_{i} d_{l} \pi_{i}+\bigoplus_{i=-1}^{k-1} \operatorname{Image} \pi_{i} d_{i}^{*} \pi_{i}+E_{k}
$$

To prove (i) we first show

$$
\bigoplus_{i=-1}^{k-1} \text { Image } \pi_{i} d_{i} \pi_{i}+E_{k} \subseteq D_{k}
$$

Clearly $E_{k} \subseteq D_{k}$ since every $w \in E_{k}$ has an extension $\omega_{\delta}$ with

$$
d_{\delta} \omega_{\delta} \in \delta^{k} \Omega[\delta] .
$$

Moreover, if $\omega \in$ Image $\pi_{i} d_{i} \pi_{l}$, then

$$
\omega=\lim _{\delta \rightarrow 0} \delta^{-i} d_{\delta} \Phi^{\prime}(v)
$$

for some $v \in \widetilde{E}_{i}$. Thus

$$
\omega_{\delta}=\delta^{-l} d_{\delta} \Phi^{\prime}(v)
$$

is an extension of $\omega$ and $d_{\delta} \omega_{\delta}=0$ so that for all $i$,

$$
\text { Image } \pi_{l} d_{i} \pi_{i} \subseteq D_{\infty} \subseteq D_{k} \text {. }
$$

To prove the converse of (2.23) we will show

$$
\left[\bigoplus_{i=-1}^{k-1} \text { Image } \pi_{i} d_{l}^{*} \pi_{i}\right] \cap D_{k}=0
$$

Suppose, for simplicity

$$
\omega \in \text { Image } \pi_{i} d_{i}^{*} \pi_{i} \cap D_{k}
$$

for some $i \leqq k-1$. Since

$$
\omega \in \text { Image } \pi_{l} d_{i}^{*} \pi_{l}
$$

we have

$$
\omega=\lim _{\delta \rightarrow 0} \delta^{-i} d_{\delta}^{*} \Phi^{\prime}(v)
$$

for some $v \in \widetilde{E}_{i}$, so that

$$
\omega_{\delta}=\delta^{-i} d_{\delta}^{*} \Phi^{\prime}(v)
$$

is an extension of $\omega$. Since $\omega \in D_{k}$, $\omega$ has another extension $\tilde{\omega}_{\delta} \in \mathscr{D}_{k}$, so that

$$
\omega_{\delta}-\tilde{\omega}_{\delta}=\delta z_{\delta}
$$

for some $z_{\delta} \in \Omega[\delta]$. Thus

$$
\begin{aligned}
0\left(\delta^{k}\right) & \ni\left\langle d_{\delta} \tilde{\omega}_{\delta}, \Phi^{\prime}(v)\right\rangle=\delta^{-i}\left\langle d_{\delta} d_{\delta}^{*} \Phi^{\prime}(v), \Phi^{\prime}(v)\right\rangle-\delta\left\langle d_{\delta} z_{\delta}, \Phi^{\prime}(v)\right\rangle \\
& =\delta^{-i}\left|d_{\delta}^{*} \Phi^{\prime}(v)\right|^{2}-\delta\left\langle z_{\delta}, d_{\delta}^{*} \Phi^{\prime}(v)\right\rangle=\delta^{\imath}|\omega|^{2}+0\left(\delta^{i+1}\right)
\end{aligned}
$$

which implies, since $i \leqq k-1, \omega=0$. The proof for general 


$$
\omega \in\left[\bigoplus_{i} \operatorname{Image} \pi_{i} d_{i}^{*} \pi_{i}\right] \cap D_{k}
$$

is the same.

Proof of (ii). The proof is by induction. $k=-1: \mathscr{D}_{-1}=\Omega[\delta]$ and $\tilde{d}_{-1}(\omega+$ $\left.\delta \omega_{1}+\cdots\right)=d^{2,-1} \omega=\pi_{-1} d_{-1} \pi_{-1} \omega$. Thus

$$
\tilde{d}_{-1} \mathscr{D}_{-1}=\text { Image } \pi_{-1} d_{-1} \pi_{-1} \text {. }
$$

General $k$ : For any $\omega_{\delta} \in \mathscr{D}_{k}, \omega_{\delta}(0) \in D_{k}$, so from part (i)

$$
\omega_{\delta}(0) \in \bigoplus_{i=-1}^{k-1} \operatorname{Image} \pi_{i} d_{i} \pi_{i}+E_{k}
$$

As seen in the proof of part (i), every $v \in$ Image $\pi_{i} d_{i} \pi_{i}$ has an extension $v_{\delta}$ with

$$
d_{\delta} v_{\delta}=0
$$

so, in particular

$$
\tilde{d}_{k} v_{\delta}=0
$$

Every $v \in E_{k}$ has an extension $v_{\delta}=\Phi^{\prime}(v) \in \mathscr{E}_{k} \subseteq \mathscr{D}_{k}$ such that

$$
\tilde{d}_{k} v_{\delta}=\pi_{k} d_{k} \pi_{k} v \text {. }
$$

Thus

$$
\tilde{d}_{k} \mathscr{D}_{k} \supseteq \text { Image } \pi_{k} d_{k} \pi_{k} \text {. }
$$

On the other hand, for any $v \in D_{k}$ the set of all extensions is precisely

$$
v_{\delta}+\delta \mathscr{D}_{k-1}
$$

where $v_{\delta}$ is the extension given above, and

$$
\tilde{d}_{k}\left(v_{\delta}+\delta \mathscr{D}_{k-1}\right)=\tilde{d}_{k} v_{\delta}+\tilde{d}_{k-1} \mathscr{D}_{k-1} \text {. }
$$

Thus

$$
\tilde{d}_{k} \mathscr{D}_{k}=\text { Image } \pi_{k} d_{k} \pi_{k}+\tilde{d}_{k-1} \mathscr{D}_{k-1} .
$$

By induction, this is equal to

$$
\bigoplus_{i=-1}^{k} \text { Image } \pi_{i} d_{i} \pi_{i}
$$

as desired

We are now prepared to prove (2.18) and (2.19)

Theorem 2.8. For every $p$ and $k$

$$
E_{k}^{p}=\bigoplus_{a+b=p} E_{k}^{a, b}
$$


Spectral Sequences and Adiabatic Limits

(ii)

$$
\begin{aligned}
& \pi_{k} d_{k} \pi_{k}\left(E_{k}^{a, b}\right) \subseteq E_{k}^{a-k+1, b+k}, \\
& \pi_{k} d_{k}^{*} \pi_{k}\left(E_{k}^{a, b}\right) \subseteq E_{k}^{a+k-1, b-k},
\end{aligned}
$$

Proof. We first note that

$$
E_{k}^{p} \subseteq \bigoplus_{a+b=p} E_{k}^{a, b}
$$

so to prove (i) it is sufficient to show that if $a+b=p$,

$$
E_{k}^{a, b} \subseteq E_{k}^{p}
$$

We will prove (i) and (ii) simultaneously inductively in $k$ $k=-1$ : Part (i). Suppose

$$
E_{-1}^{p} \ni \omega=\sum_{a+b=p} \omega^{a, b}
$$

where

$$
\omega^{a, b} \in \Omega^{a, b} .
$$

We need to see that for all $a$ and $b$,

$$
\omega^{a, b} \in E_{-1}^{p} .
$$

Since $\omega \in E_{-1}^{p}$ we have

$$
d^{2,-1} \omega=\left(d^{2,-1}\right)^{*} \omega=0,
$$

but

$$
0=d^{2,-1} \omega=\sum_{a, b} d^{2,-1} \omega^{a, b}
$$

Since

$$
d^{2,-1} \omega^{a, b} \in \Omega^{a+2, b-1},
$$

(2.20) implies that for all $a$ and $b$

$$
d^{2,-1} \omega^{a, b}=0 .
$$

Similarly,

$$
\left(d^{2,-1}\right)^{*} \omega=0
$$

implies that for all $a$ and $b$

$$
\left(d^{2,-1}\right)^{*} \omega^{a, b}=0
$$

and thus

$$
\omega^{a, b} \in E_{-1}^{P} .
$$

Part (ii). We note simply that for

$$
\omega^{a, b} \in E_{-1}^{a, b},
$$

We have

$$
\pi_{-1} d_{-1} \pi_{-1} \omega^{a, b}=d^{2,-1} \omega^{a, b} \in E_{-1}^{a+2, b-1} .
$$

Similarly

$$
\pi_{-1} d_{-1}^{*} \pi_{-1} \omega^{a, b}=\left(d^{2,-1}\right)^{*} \omega^{a, b} \in E_{-1}^{a-2, b-+1}
$$


as desired

General $k$ : Part (i). Suppose

$$
\omega^{p}=\sum_{a+b=p} \omega^{a, b} \in E_{k}^{p} .
$$

Then certainly $\omega \in E_{k-1}^{p}$, so by induction on part (i)

$$
\omega^{a, b} \in E_{k-1}^{p} .
$$

For any $v \in E_{k-1}^{p}$,

$$
v \in E_{k}^{p} \longleftrightarrow \pi_{k-1} d_{k-1} \pi_{k-1} v=\pi_{k-1} d_{k-1}^{*} \pi_{k-1} v=0 .
$$

But $\omega \in E_{k}^{p}$ implies

$$
0=\pi_{k-1} d_{k-1} \pi_{k-1} w=\sum_{a, b} \pi_{k-1} d_{k-1} \pi_{k-1} \omega^{a, b} .
$$

By induction on part (ii),

$$
\pi_{k-1} d_{k-1} \pi_{k-1} \omega^{a, b} \in \Omega^{a-k+2, b+k-2} .
$$

So that (2.21) implies

$$
\pi_{k-1} d_{k-1} \pi_{k-1} \omega^{a, b}=0
$$

Similarly

$$
\pi_{k-1} d_{k-1}^{*} \pi_{k-1} \omega=0
$$

implies that

$$
\pi_{k-1} d_{k-1}^{*} \pi_{k-1} \omega^{a, b}=0 .
$$

Together, (2.22) and (2.23) imply that for all $a$ and $b$,

$$
\omega^{a, b} \in E_{k}^{p}
$$

as desired.

Part (ii). Suppose $\omega^{a, b} \in E_{k}^{a, b}$ so that there exists a polynomial

$$
\omega_{\delta}=\omega^{a, b}+\delta \omega_{1}+\delta^{2} \omega_{2}+\cdots
$$

with

$$
d_{\delta} \omega_{\delta}, d_{\delta}^{*} \omega_{\delta} \in \delta^{k} \Omega[\delta]
$$

Then

$$
\begin{aligned}
d_{\delta} \omega_{\delta} & =d_{\delta}\left(\omega^{a, b}+\sum_{l>0} \delta^{l} \omega_{i}\right) \\
& d_{\delta}\left(\omega^{a, b}+\sum_{i>0} \delta^{i} \omega_{i}^{a-i, b+1}\right)+\sum_{c \neq 0} d_{\delta}\left(\sum_{i>0} \delta^{l} \omega_{i}^{a+c-l, b-c+l}\right) .
\end{aligned}
$$

Let

$$
\tilde{\omega}_{\delta}=\omega^{a, b}+\sum_{l>0} \delta^{i} \omega_{i}^{a-i, b+i} .
$$

The first term on the right-hand side of (2.24) is equal to 


$$
d_{\delta} \tilde{\omega}_{\delta}=\delta^{-1}\left(d^{2,-1} \omega^{a, b}\right)+\left(d^{1,0} \omega^{a, b}+d^{2,-1} \omega_{1}^{a-1, b+1}\right)+\cdots .
$$

Writing, for any $v_{\delta} \in \Omega[\delta]$,

$$
d_{\delta} v_{\delta}=\delta^{-1} \tilde{d}_{-1} v_{\delta}+\tilde{d}_{0} v_{\delta}+\delta \tilde{d}_{1} v_{\delta}+\cdots .
$$

we see that

$$
\tilde{d}_{j} \tilde{\omega}_{\delta} \in \Omega^{a-j+1, b+j} .
$$

Similarly, for all $c \neq 0$,

$$
\tilde{d}_{j}\left(\sum_{i>0} \delta^{i} \omega_{i}^{a+c-l, b-c+i}\right) \in \Omega^{a+c-j+1, b-c+j-1} .
$$

Since $d_{\delta} \omega_{\delta} \in 0\left(\delta^{k}\right)$ we have that for $j<k$,

$$
0=\tilde{d}_{j} \omega_{\delta}=\tilde{d}_{j} \tilde{\omega}_{\delta}+\sum_{c} \tilde{d}_{j}\left(\sum_{i>0} \delta^{i} \omega_{i}^{a+c-l, b-c,+i}\right) .
$$

From (2.25) and (2.26) we learn that for $j<k$ and every $c \neq 0$,

$$
0=\tilde{d}_{j} \tilde{\omega}_{\delta}=\tilde{d}_{j}\left(\sum_{i>0} \delta^{l} \omega_{i}^{a+c-l, b-c+i}\right) .
$$

In particular, for $c \neq 0$,

$$
\sum_{i>0} \delta^{i} \omega^{a+c-l, b-c+i} \in \mathscr{D}_{k}
$$

which implies

$$
\sum_{l>0} \delta^{i-1} \omega_{i}^{a+c-i, b-c+i} \in \mathscr{D}_{k-1} .
$$

Therefore, by Lemma 2.7, for $c \neq 0$,

$$
\begin{aligned}
\tilde{d}_{k}\left(\sum_{i>0} \delta^{i} \omega_{i}^{a+c-i, b-c+l}\right) & =\tilde{d}_{k-1}\left(\sum_{i>0} \delta^{i-1} \omega_{i}^{a+c-i, b-c+i}\right) \\
& \in \bigoplus_{i=-i}^{k-1} \operatorname{Image} \pi_{i} d_{i} \pi_{i} \subseteq E_{k}^{\perp} .
\end{aligned}
$$

By induction on part (i), for $i<k$,

$$
E_{i}^{p}=\bigoplus_{a+b=p} E_{i}^{a, b}
$$

So that for $i<k$, and every $a$ and $b$,

$$
v \in \Omega^{a, b} \Longrightarrow \tilde{\pi}_{i} v \in \Omega^{a, b} .
$$

Together, (2.25), (2.27) and (2.28) imply

$$
\pi_{k} d_{k} \pi_{k} \omega^{a, b}=\pi_{k} \tilde{d}_{k} \omega_{\delta}=\pi_{k} \tilde{d}_{k} \tilde{\omega}_{\delta}=\tilde{d}_{k} \tilde{\omega}_{\delta}-\sum_{i<k} \tilde{\pi}_{i} \tilde{d}_{k} \tilde{\omega}_{\delta} \in \Omega^{a-k+1, b+k} .
$$


The same argument shows

$$
\pi_{k} d_{k}^{*} \pi_{k} \omega^{a, b} \in \Omega^{a+k-1, b-k},
$$

as desired.

\section{The Convergence of the Sequence $\left\{E_{k}^{p}\right\}$}

We now describe the sense in which this spectral sequence converges to the cohomology of $M$ with values in $V$. (This section essentially follows the ideas of Sects. 2, 5 and 6 of [Ma-Me]).

In Theorem 3.3 we prove that the space of formal Laurent series in $\delta$ with values in $\Omega^{p}(V)$ has a Hodge decomposition

$$
\left(d_{\delta} \text { exact }\right) \oplus\left(d_{\delta} \text { coexact }\right) \oplus\left(d_{\delta} \text { harmonic }\right) .
$$

This implies that the space of harmonic Laurent series is isomorphic to the Laurent cohomology

$$
\left(d_{\delta} \text { closed Laurent series }\right) /\left(d_{\delta} \text { exact Laurent series }\right)
$$

This isomorphism is as modules over $\mathscr{L}$, the space of real formal Laurent series.

On the one hand, we observe (3.4) that the Laurent cohomology is isomorphic to the space of Laurent series with values in the usual deRham cohomology $H^{p}(M, V)$.

On the other hand we show (Theorem 3.1) that every $v \in E_{\infty}^{p}$ has an extension to a formal power series which is formally harmonic, and that these form a basis, modulo $\mathscr{L}$, of the harmonic Laurent series.

Thus, combining the above observations, we have, in particular, that

$$
\operatorname{dim} E_{\infty}^{p}=\operatorname{dim} H^{p}(M, V) .
$$

In Sect. 5 of this paper we show, with some additional geometric hypotheses, that as $\delta \rightarrow 0$ the space of $g_{\delta}$ harmonic forms approaches the space $E_{\infty}^{p}$.

We begin by proving that every $v \in E_{\infty}^{p}$ is the value at $\delta=0$ of a formally harmonic power series.

Theorem 3.1. Suppose $v \in E_{\infty}^{p}$. Then there is a unique formal power series

$$
v_{\delta}=v+\delta v_{1}+\delta^{2} v_{2}+\delta^{3} v_{3}+\ldots
$$

such that

$$
v_{\imath} \perp E_{\infty}^{p} \quad \text { for all } i \geqq 1
$$

and, formally,

$$
d_{\delta} v_{\delta}=d_{\delta}^{*} v_{\delta}=0
$$

Proof. Uniqueness: If $v_{\delta}$ and $v_{\delta}^{\prime}$ are two power series of the form (3.1), then $v_{\delta}-v_{\delta}^{\prime}$ is a formal power series satisfying (3.3). Thus, the first non-zero coefficient must be in $E_{\infty}^{p}$. On the other hand all coefficients of $v_{\delta}-v_{\delta}^{\prime}$ are in $\left(E_{\infty}^{p}\right)^{\perp}$. Thus $v_{\delta}-v_{\delta}^{\prime}$ must be 0 .

Existence: For every $v \in E_{\infty}^{p}$ and every $K>N$, there is a polynomial

$$
v_{\delta, K}=v+\delta v_{1}+\cdots+\delta^{j} v_{j}
$$


such that

$$
d_{\delta} v_{\delta}, d_{\delta}^{*} v_{\delta} \in 0\left(\delta^{K}\right)
$$

The form $v_{1}$ is not uniquely determined since we can add any form-valued polynomial $\delta \omega_{\delta}$ with $\omega_{\delta} \in \mathscr{E}_{K-1}$. Any such polynomial must satisfy $\omega_{0} \in E_{K-1}^{p}=E_{N}^{p}=$ $E_{\infty}^{p}$. Thus, the non-uniqueness of $v_{1}$ is given precisely by $E_{N}^{p}$. Hence we can choose a $v_{\delta, K}$ with $v_{1} \in E_{\infty}^{\perp}$. This value of $v_{1}$ is unique and independent of $K$. If $K>N+1$, then our choice of $v_{2}$ is again determined up to $E_{\infty}$, so we can choose $v_{\delta, K}$ with $v_{1}, v_{2} \in E_{\infty}^{\perp}$. Continuing in this fashion, we can find a $v_{\delta, K}$ with $v_{1}, \ldots, v_{K-N} \in E_{\infty}^{\perp}$.

These forms are uniquely determined. For fixed $j$, the values of $v_{i}, 1 \leqq i \leqq j$ are fixed for $K \geqq N+j$. Hence, as $K \rightarrow \infty$, the $v_{\delta, K}$ chosen in this fashion converge to a power series satisfying (3.2) and (3.3).

For simplicity, it is useful to introduce the notion of a Laurent series of forms. Define $\mathscr{L}\left[\Omega^{p}\right]$ to be the space of Laurent series with coefficients in $\Omega^{p}$, i.e. and element of $\mathscr{L}\left[\Omega^{p}\right]$ is of the form

$$
v_{\delta}=\sum_{j \geqq a} \delta^{j} v_{j}
$$

with $a \in \mathbf{Z}$ and $v_{j} \in \Omega^{p}$ for all $j$. The operator $d_{\delta}$ maps $\mathscr{L}\left[\Omega^{p}\right]$ to $\mathscr{L}\left[\Omega^{p+1}\right]$ and satisfies $d_{\delta}^{2}=0$. Thus we can define, for $0 \leqq p \leqq \operatorname{dim} M$,

$$
H_{\mathscr{L}}^{p}=Z_{\mathscr{L}}^{p} / B_{\mathscr{L}}^{p}
$$

where $Z_{\mathscr{L}}^{p}$ is the kernel of the map

$$
d_{\delta}^{p}: \mathscr{L}\left[\Omega^{p}\right] \rightarrow \mathscr{L}\left[\Omega^{p+1}\right]
$$

and $B_{\mathscr{L}}^{p}$ is the image of $d_{\delta}^{p-1}$. At first it is simpler to consider instead the space

$$
\bar{H}_{\mathscr{L}}^{p}=\bar{Z}_{\delta}^{p} / \bar{B}_{\delta}^{p}
$$

where

$$
\bar{Z}_{\mathscr{L}}^{p}=\text { kernel of } d \subset \mathscr{L}\left[\Omega^{p}\right],
$$

where $d$ is the usual $d$ operator acting term by term on Laurent series, and

$$
\bar{B}_{\mathscr{L}}^{p}=\text { image of } d \subset \mathscr{L}\left[\Omega^{p}\right] .
$$

Clearly

$$
\bar{Z}_{\mathscr{L}}^{p}=\mathscr{L}\left[Z^{p}\right]
$$

where $Z^{p} \subset \Omega^{p}$ is the kernel of $d$, i.e. the usual space of closed forms, and $\mathscr{L}\left[Z^{p}\right]$ is the space of Laurent series with coefficients in $Z$.

Similarly,

$$
\bar{B}_{\mathscr{L}}^{p}=\mathscr{L}\left[B^{p}\right]
$$

where $B^{p} \subset \Omega^{p}$ is the image of $d$, i.e. the usual space of exact forms. Thus

$$
\bar{H}_{\mathscr{L}}^{p}=\bar{Z}_{\delta}^{p} / \bar{B}_{\delta}^{p} \cong \mathscr{L}\left[H^{p}(M, V)\right] .
$$

Note that $\bar{H}_{\mathscr{L}}^{p}$ is an $\mathscr{L}$ denotes the ring of formal Laurent series with real coefficients, isomorphic (as $\mathscr{L}$-modules) to

$$
H^{p}(M, V) \otimes_{\mathbf{R}} \mathscr{L}
$$


So that, in particular,

$$
\operatorname{dim}_{\mathscr{L}} \bar{H}_{\mathscr{L}}^{p}=\operatorname{dim}_{\mathbf{R}} H^{p}(M, V) .
$$

Now observe that the map $\rho_{\delta}$ defined in Sect. 1 induces an isomorphism

$$
\rho_{\delta}: \mathscr{L}\left[\Omega^{p}\right] \rightarrow \mathscr{L}\left[\Omega^{p}\right] .
$$

Moreover, $\rho_{\delta}$ maps $\bar{Z}_{\delta}^{p}$ to $Z_{\delta}^{p}, \bar{B}_{\delta}^{p}$ to $B_{\delta}^{p}$, and commutes with the action of $\mathscr{L}$. Thus $\rho_{\delta}$ induces an isomorphism as $\mathscr{L}$ modules

$$
\rho_{\delta}: \bar{H}_{\mathscr{L}}^{p} \rightarrow H_{\mathscr{L}}^{p} .
$$

Our goal now is to relate the space $E_{\infty}^{p}$ to $H_{\mathscr{L}}^{p}$. From Lemma 3.1 there is a map

$$
v \in E_{\infty}^{p} \mapsto v_{\delta}=v+\delta v_{1}+\delta^{2} v_{2}+\ldots
$$

with $v_{\delta}$ satisfying (3.2) and (3.3). The linearity of the map $v \mapsto v_{\delta}$ follows from the uniqueness of $v_{\delta}$. That is, if $v_{1}+v_{2}=v$, then $\left(v_{1}\right)_{\delta}+\left(v_{2}\right)_{\delta}$ satisfies (3.2) and (3.3) so we must have

$$
\left(v_{1}\right)_{\delta}+\left(v_{2}\right)_{\delta}=v_{\delta} .
$$

For $v \in E_{\infty}^{p}$, we have $d_{\delta} v_{\delta}=0$, so $\left[v_{\delta}\right]$ represents a class in $H_{\mathscr{L}}^{p}$. This map extends to a map

$$
E_{\infty}^{p} \otimes \mathscr{L} \rightarrow H_{\mathscr{L}}^{p}
$$

where the element

$$
\sum_{j \geqq a} \delta^{j} v_{j} \in E_{\infty}^{p} \otimes \mathscr{L}
$$

(so that $v_{j} \in E_{\infty}^{p}$ for all $j$ ), is mapped to the element

$$
\sum_{j \geqq a} \delta^{j}\left[\left(v_{j}\right)_{\delta}\right] \in H_{\mathscr{L}}^{p}
$$

In fact, the map (3.5) is an isomorphism. This will be proved in 2 steps

(i) The map (3.5) is an injection:

This follows from the following lemma

Lemma 3.2. If $w \in \mathscr{L}[\Omega]$ satisfies

$$
d_{\delta}^{*} d_{\delta} w=0
$$

then

$$
d_{\delta} w=0
$$

Proof. Suppose $w=\sum_{j \geqq a} \delta^{j} w_{j}$, and let

$$
w^{k}=\sum_{a \leqq j \leqq k} \delta^{j} w_{j} .
$$

Then

$$
d_{\delta}^{*} d_{\delta} w^{k}=d_{\delta}^{*} d_{\delta}\left(w^{k}-w\right) \in \delta^{k-1} \Omega^{p}[[\delta]],
$$

so that

$$
\left\langle d_{\delta} w^{k}, d_{\delta} w^{k}\right\rangle=\left\langle d_{\delta}^{*} d_{\delta} w^{k}, w^{k}\right\rangle \in \delta^{k+a-1} \Omega^{p}[[\delta]] .
$$


This implies

$$
d_{\delta} w^{k} \in \delta^{\left[\frac{k+a-1}{2}\right]} \Omega^{p}[[\delta]] .
$$

We also know

$$
d_{\delta} w-d_{\delta} w^{k} \in \delta^{k} \Omega^{p}[[\delta]] .
$$

It follows from (3.6) and (3.7) that

$$
d_{\delta} w \in \delta^{l(k)} \Omega^{p}[[\delta]],
$$

where

$$
l(k)=\min \left\{\left[\frac{k+a-1}{2}\right], k\right\}=\left[\frac{k+a-1}{2}\right] \text { for } k \text { large enough } .
$$

Letting $k \rightarrow \infty$ proves the lemma.

If $v_{\delta}$ is harmonic and $v_{\delta}=d_{\delta} w_{\delta}$, then

$$
0=d_{\delta}^{*} v_{\delta}=d_{\delta}^{*} d_{\delta} w_{\delta}
$$

which implies, by Lemma 3.2, that

$$
v_{\delta}=d_{\delta} w_{\delta}=0 .
$$

Thus, for $0 \neq v \in E_{\infty}^{p},\left[v_{\delta}\right] \in H_{\mathscr{L}}^{p}$ is non-zero, so the map (3.5) is an injection.

(ii) The map (3.5) is a surjection: forms:

This follows from the existence of a Hodge decomposition for Laurent series of

Theorem 3.3. If $v \in \mathscr{L}\left[\Omega^{p}\right]$ then there exist $v_{1}, v_{2}, v_{3} \in \mathscr{L}\left[\Omega^{p}\right]$ such that

a) $v_{1}$ is harmonic,

b) $v_{2} \in d_{\delta} \mathscr{L}\left[\Omega^{p-1}\right]$,

c) $v_{3} \in d_{\delta}^{*} \mathscr{L}\left[\Omega^{p+1}\right]$,

d) $v=v_{1}+v_{2}+v_{3}$.

Moreover, $v_{1}, v_{2}$ and $v_{3}$ are uniquely determined by $v$. (Note that if $d_{\delta} v=0$ then we have $v_{3}=0$ by Lemma 3.2, and this proves (3.5) is a surjection).

Proof. The uniqueness of the $v_{i}$ is clear. To prove existence we work modulo harmonic Laurent series.

Write

$$
v=\sum_{j \geqq a} \delta^{j} v_{j} .
$$

By adding the harmonic series $-\delta^{a}\left(\pi_{\infty} v_{a}\right)_{\delta}$ to $v$ we can assume $v_{a} \in E_{\infty}^{\perp}$. Then by adding $-\delta^{a+1}\left(\pi_{\infty} v_{a+1}\right)_{\delta}$ to $v$ we can assume $c_{a+1} \in E_{\infty}^{\perp}$. Continuing in this fashion, modulo harmonic series we can write

$$
v=\sum_{j \geqq a} \delta^{j} \sum_{k=-1}^{N-1} v_{j, k}
$$

with $v_{j, k} \in \tilde{E}_{k}^{p}$ for all $j, k$. 
We define a linear ordering on the pairs $(j, k), j \in \mathbf{Z},-1 \leqq k \leqq N-1$, as follows:

Say $(j, k)<\left(j^{\prime}, k^{\prime}\right)$ if and only if

$$
j+k<j^{\prime}+k^{\prime} \text { or } j+k=j^{\prime}+k^{\prime} \text { and } j<j^{\prime} .
$$

Let $\left(j_{0}, k_{0}\right)=\min \left\{(j, k) \mid v_{j, k} \neq 0\right\}$. From Theorem 2.5(b), we know that

$$
\Delta_{k_{0}}: \tilde{E}_{k_{0}}^{p} \rightarrow \tilde{E}_{k_{0}}^{p}
$$

is an isomorphism. Therefore there exist $w_{1} \in \tilde{E}_{k_{0}}^{p-1}, w_{2} \in \tilde{E}_{k_{0}}^{p+1}$ such that

$$
v_{j_{0}, k_{0}}=d_{k_{0}} w_{1}+d_{k_{0}}^{*} w_{2}
$$

Let

$$
v^{\prime}=v-d_{\delta}\left(\delta^{j_{0}} \Phi^{\prime}\left(w_{1}\right)\right)-d_{\delta}^{*}\left(\delta^{j_{0}} \Phi^{\prime}\left(w_{2}\right)\right) .
$$

As before, modulo harmonic series we can write

$$
v^{\prime}=\sum_{j \geqq a} \delta^{j} \sum_{k=-1}^{N-1} v_{j, k}^{\prime}
$$

with $v_{j, k}^{\prime} \in \tilde{E}_{k}$ for all $j, k$. Formula (3.8) implies $v_{j_{0}, k_{0}}^{\prime}=0$. Corollary 2.6 implies that if

$$
\left(j_{1}, k_{1}\right)=\min \left\{(j, k) \mid v_{j, k}^{\prime} \neq 0\right\},
$$

then $\left(j_{1}, k_{1}\right)>\left(j_{0}, k_{0}\right)$. Thus we can repeat the operation and in this fashion construct the desired $v_{2}$ and $v_{3}$ term by term.

\section{The Leray Spectral Sequence}

In this section we show that the spectral sequence $\left\{E_{k}^{p}, \pi_{k} d_{k} \pi_{k}\right\}$, or more precisely $\left\{\mathscr{L}\left(E_{k}^{p}\right), \pi_{k} d_{k} \pi_{k}\right\}$, is isomorphic to the Leray spectral sequence associated to a filtered differential complex constructed from $M$ and the splitting $T M=A+B$. As a corollary, we learn that for all $p$ and $k$ the dimension of $E_{k}^{p}$ is independent of the metric $g=g_{A}+g_{B}$.

In addition, in the case that $A$ is integreable, we show that $\left\{E_{k}^{p}, \pi_{k} d_{k} \pi_{k}\right\}$ is isomorphic to the standard Leray spectral sequence associated to the corresponding foliation.

Let us briefly recall Leray's construction of a spectral sequence from a filtered differential complex. (See [McC] Sect. 2.2 for general discussion.)

Let $\{K, \partial\}$ be a differential complex. That is,

$$
K=\bigoplus_{i} K^{i}, \partial\left(K^{i}\right) \subseteq K^{i+1} \quad \text { and } \quad \partial^{2}=0 .
$$

Suppose further that

$$
K=\cdots \supseteq K_{-1} \supseteq K_{0} \supseteq K_{1} \supseteq \ldots
$$


is a filteration by subcomplexes, i.e.

$$
K=\bigcup_{i} K_{i} \quad \text { and } \quad \partial\left(K_{l}\right) \subseteq K_{i}
$$

Then we get a spectral sequence as follows: Define

$$
\begin{aligned}
& Z_{r}^{p, q}=K_{p}^{p+q} \cap \partial^{-1}\left(K_{p+r}^{p+q+1}\right), \\
& B_{r}^{p, q}=K_{p}^{p+q} \cup \partial\left(K_{p-r}^{p+q+1}\right),
\end{aligned}
$$

and

$$
e_{r}^{p, q}=Z_{r}^{p, q} / Z_{r-1}^{p+1, q-1}+B_{r-1}^{p, q} .
$$

Then ([McC] Theorem 2.1) the operator $\partial$ induces a differential $\partial_{r}$ on the spaces $e_{r}^{p, q}$ such that

$$
e_{r+1}=H^{*}\left(e_{r}, \partial_{r}\right)
$$

and thus the complexes $\left\{e_{r}, \partial_{r}\right\}$ form a spectral sequence. Lastly, we define

$$
e_{r}^{l}=\bigcup_{\alpha} \bigoplus_{p \geqq \alpha} e_{r}^{p, l-p},
$$

so that

$$
\partial_{r}: e_{r}^{l} \rightarrow e^{l+1}
$$

To place the spectral sequence $\left\{E_{k}^{p}, \pi_{k} d_{k} \pi_{k}\right\}$ in this general framework, we borrow an idea from Sect. 3 and let

$$
K^{p}=\mathscr{L}\left[\Omega^{p}\right]
$$

where $\mathscr{L}\left[\Omega^{p}\right]$ denotes the space of Laurent series in $\delta$ with coefficients in $\Omega^{p}$. Let

$$
K_{l}^{p}=\delta^{i} \Omega^{p}[[\delta]]
$$

so that

$$
K^{p}=\cdots \supseteq K_{-1}^{p} \supseteq K_{0}^{p} \supseteq K_{1}^{p} \supseteq \cdots .
$$

For the differential, we take

$$
\partial=\delta d_{\delta}=d^{2,-1}+\delta d^{1,0}+\delta^{2} d^{0,1}+\delta^{3} d^{-1,2},
$$

so that

Note also that

$$
\partial\left(K_{l}\right) \subseteq K_{l}
$$

$$
H^{p}(K, \partial)=H_{p}\left(K, d_{\delta}\right) \cong \mathscr{L}\left[E_{\infty}^{p}\right]
$$

In fact, we have

Theorem 4.1. With $K_{l}$ and $\partial$ defined as in (4.1), (4.2) and (4.3), the induced spectral sequence satisfies:

For every $r$

$$
\left\{e_{r}, \partial_{r}\right\} \cong\left\{\mathscr{L}\left[E_{r-1}\right], \pi_{r-1} d_{r-1} \pi_{r-1}\right\},
$$

where $\pi_{r-1} d_{r-1} \pi_{r-1}$ acts term by term on $\mathscr{L}\left[E_{r-1}\right]$.

Proof. We begin by defining the space

$$
\overline{\mathscr{D}}_{k}^{p}=\left\{\omega_{\delta} \in \Omega^{p}[[\delta]] \mid d_{\delta} \omega_{\delta} \in \delta^{k} \Omega^{p+1}[[\delta]]\right\} .
$$

Note that $\overline{\mathscr{D}}_{k}^{p}$ is just a completion of the space $\mathscr{D}_{k}^{p}$ defined in (2.20). 
Then

$$
\begin{aligned}
& Z_{r}^{p, q}=\delta^{p} \Omega^{p+q}[[\delta]] \cap \partial^{-1}\left(\delta^{p+r} \Omega^{p+q+1}[[\delta]]\right)=\delta^{p} \overline{\mathscr{D}}_{r-1}^{p+q}, \\
& B_{r}^{p, q}=\delta^{p} \Omega^{p+q}[[\delta]] \cap \partial\left(\delta^{p-r} \Omega^{p+q-1}[[\delta]]\right)=\delta^{p-r} \partial \overline{\mathscr{D}}_{r-1}^{p+q-1},
\end{aligned}
$$

and

$$
\begin{aligned}
e_{r}^{p, q} & =\frac{Z_{r}^{p, q}}{Z_{r-1}^{p+1, q+1}+B_{r-1}^{p, q}}=\frac{\delta^{p} \overline{\mathscr{D}}_{r-1}^{p+q}}{\delta^{p+1} \overline{\mathscr{D}}_{r-2}^{p+q}+\delta^{p-r+1} \partial \overline{\mathscr{D}}_{r-2}^{p+q-1}}, \\
& \cong \delta^{p}\left[\frac{D_{r-1}^{p+q}}{\tilde{d}_{r-1} \overline{\mathscr{D}}_{r-2}^{p+q-1}}\right]
\end{aligned}
$$

(where $D_{r-1}^{p+q}$ and $\tilde{d}_{r-2}$ are defined in (2.21) so that

$$
e_{r}^{l}=\bigcup_{\alpha} \bigoplus_{p \geqq \alpha} e_{r}^{p, l-p} \cong \mathscr{L}\left[\frac{D_{r}^{l}}{\tilde{d}_{r-2} \overline{\mathscr{D}}_{r-2}^{l-1}}\right] .
$$

The theorem follows from the following two facts:

(i) For all $l$ and $r$

$$
D_{r}^{l}=\bigoplus_{i=-1}^{r-1} \operatorname{Image}\left(\left.\pi_{i} d_{i} \pi_{i}\right|_{E_{l}^{l-1}}\right)+E_{r}^{l} .
$$

(ii) For all $l$ and $r$

$$
\tilde{d}_{r}\left(\overline{\mathscr{D}}_{r}^{l-1}\right)=\bigoplus_{i=-1}^{r} \operatorname{Image}\left(\left.\pi_{i} d_{i} \pi_{i}\right|_{E_{i}^{l-1}}\right)
$$

which is precisely the content of Lemma 2.7. (The slight difference between $\mathscr{D}_{r}^{k}$ and $\overline{\mathscr{D}}_{r}^{k}$ does not affect the lemma or the proof).

Now, we note that (i) and (ii), combined with (4.5) clearly yield

$$
e_{r}^{l} \cong \mathscr{L}\left[E_{r-1}^{l}\right] \text {. }
$$

Moreover, the correspondence between $\partial_{r}$ and $\pi_{r-1} d_{r-1} \pi_{r-1}$ follows as well:

For a class $\alpha \in e_{r}^{p, q}$, let $\delta^{p} \bar{\alpha} \in \delta^{p} \mathscr{D}_{r-1}^{p=q}$ be any representative of $\alpha$ (using the formulation (4.5)), and $\bar{\alpha}_{\delta} \in \overline{\mathscr{D}}_{r-1}^{p+q}$ any extension of $\bar{\alpha}$.

Then $\delta^{p} \bar{\alpha}_{\delta}$ represents $\alpha$ in (4.4), and by definition

$$
\partial_{r} \alpha=\left[\partial \delta^{p} \bar{\alpha}_{\delta}\right]
$$

where $\left[\partial \delta^{p} \bar{\alpha}_{\delta}\right]$ denotes the class in $e_{r}^{p+1, q-1}$ represented by $\partial \delta^{p} \bar{\alpha}_{\delta}$. From (i) and (ii) there is a unique $\bar{\alpha} \in \delta^{p} E_{r-1}^{p+q}$ which represents $\alpha$. We can then choose $\bar{\alpha}_{\delta}=\Phi^{\prime}(\alpha)$ (where $\Phi^{\prime}$ is the extension defined in Theorem 2.4), so that

$$
\partial_{r} \alpha=\left[\delta^{p} \partial \bar{\alpha}_{\delta}\right]=\left[\delta^{p+1} d_{\delta} \Phi^{\prime}(\bar{\alpha})\right]=\left[\delta^{p+r} \pi_{r-1} d_{r-1} \pi_{r-1} \bar{\alpha}\right]
$$

as claimed. 
The spectral sequence $\left\{e_{k}^{p}, \partial_{k}\right\}$ is defined independently of the metric $g=$ $g_{A}+g_{B}$. Moreover, it is clear from the proof of Theorem 4.1 that the isomorphism

$$
\left(e_{k}^{p}, \partial_{k}\right) \cong\left(\mathscr{L}\left[E_{k-1}^{p}\right], \pi_{k-1} d_{k-1} \pi_{k-1}\right)
$$

is as $\mathscr{L}$-modules. In particular, we learn

Corollary 4.2. For all $p$ and $k, \operatorname{dim} E_{k}^{p}$ is independent of the metrics $g_{A}$ and $g_{B}$ in the decomposition

$$
g=g_{A}+g_{B}
$$

For the remainder of this section we assume that $A$ is integrable. In this case we know that

$$
d^{2,-1}=0
$$

so that, in particular

$$
d=d^{1,0}+d^{0,1}+d^{-1,2} .
$$

This implies that our spectral sequence satisfies

$$
\Omega^{*}=E_{-1}=E_{0} .
$$

There is a canonical filtration of the deRham complex associated to an integrable distribution. Namely, let

$$
K=\Omega^{*}(V)
$$

and

$$
K_{i}=\bigoplus_{\substack{a \in z \\ b \geqq 1}} \Omega^{a, b}(V)
$$

Then

$$
K=K_{0} \supseteq K_{1} \supseteq \cdots \supseteq K_{\operatorname{dim} B} \supseteq K_{\operatorname{dim} B+1}=0
$$

and (4.7) shows

$$
d\left(K_{i}\right) \subseteq K_{i} .
$$

This data gives rise to a Leray spectral sequence defined by (4.1), (4.2) and (4.3).

In analogy with our earlier analysis, to help identify the spaces $e_{r}^{p, q}$ we define

$$
\mathscr{D}_{r}^{p, q}=\left\{\omega \in \Omega^{p+q} \cap K_{q} \mid d \omega \in \Omega^{p+q+1} \cap K_{q+r}\right\}
$$

and

$$
\mathscr{D}_{r}^{p, q}=\left\{\omega \in \Omega^{p, q} \mid \tilde{\omega} \in \mathscr{D}_{r}^{p, q} \text { with } \pi^{p, q} \tilde{\omega}=\omega\right\} .
$$

We also define the operator

$$
\tilde{d}_{r}: \mathscr{D}_{r}^{p, q} \rightarrow D_{r}^{p-r+1, q+r}
$$

by

$$
\tilde{d}_{r} \omega: \pi^{p-r+1, q+r} d \omega .
$$

(Note that

$$
d(d \omega)=0 \quad \text { and } \quad(d \omega) \in K_{q+r} \cap \Omega^{p+q+1}
$$

imply

$$
\left.\pi^{p-r+1, q+r}(d \omega) \in D^{p-r+1, q+r} \subseteq D_{r}^{p-r+1, q+r}\right) .
$$


Then

$$
e_{r}^{p, q} \cong D_{r}^{p, q} / \tilde{d}_{r-1} \mathscr{D}_{r-1}^{p+r-2, q-r+1}
$$

The expression on the right-hand side of (4.8) can be simplified with the following lemma

Lemma 4.3. For all $p, q$ and $r$

$$
D_{r}^{p, q}=\left[\bigoplus_{i=0}^{r-1}\left(\text { Image } \pi_{i} d_{i} \pi_{i} \cap \Omega^{p, q}\right)\right] \oplus E_{r}^{p, q},
$$

$$
\tilde{d}_{r} \mathscr{D}_{r}^{p, q}=\left[\bigoplus_{i=0}^{r}\left(\text { Image } \pi_{i} d_{i} \pi_{i} \cap \Omega^{p-r+1, q+r}\right)\right] .
$$

Before proving this lemma we state two corollaries. Lemma 4.3 and (4.8) imply

$$
e_{r}^{p, q} \cong E_{r}^{p, q} \text {. }
$$

Moreover, the proof of (4.6) can easily be adapted to our context to prove that the differentials coincide. Thus we have

Corollary 4.4. If $A$ is integrable

$$
\left\{e_{r}^{p, q}, \partial_{r}\right\} \cong\left\{E_{r}^{p, q}, \pi_{r} d_{r} \pi_{r}\right\},
$$

where $\left\{e_{r}^{p, q}, \partial_{r}\right\}$ is the Leray spectral sequence associated with $A$

We note that the Leray spectral sequence $\left\{e_{r}^{p, q}, \partial_{r}\right\}$ is defined without reference to the metric $g=g_{A}+g_{B}$. Moreover, it can be defined without reference to the distribution $B$. This can be done by observing

$$
\begin{aligned}
K_{i}= & \bigoplus_{p}\left\{\omega \in \Omega^{p} \mid i_{a_{1}} i_{a_{2}} \ldots i_{a_{p-l+1}} \omega=0 \text { (where } i=\right.\text { interior product } \\
& \text { for all } \left.a_{1}, \ldots, a_{p-i+1} \in A\right\} .
\end{aligned}
$$

This implies

Corollary 4.5. If $A$ is integrable then for all $p, q$ and $r$

$$
\operatorname{dim} E_{r}^{p, q}
$$

depends only on the distribution A. In particular, $\operatorname{dim} E_{r}^{p, q}$ is independent of the complementary distribution $B$ as well as the metrices $g_{A}$ and $g_{B}$.

Thus it only remains to prove Lemma 4.3

Proof of Lemma 4.3:

(i) We first show

$$
\left[\bigoplus_{l=0}^{r-1}\left(\text { Image } \pi_{l} d_{i} \pi_{i} \cap \Omega^{p, q}\right)\right] \oplus E_{\subseteq}^{p, q} D_{r}^{p, q} .
$$

To prove $E_{r}^{p, q} \subseteq D_{r}^{p, q}$ we let

$$
\omega^{p, q} \in E_{r}^{p, q} .
$$


Then there exist $\omega_{1}, \omega_{2}, \ldots$ with

$$
d_{\delta}\left(\omega^{p, q}+\delta \omega_{1}+\delta^{2} \omega_{2}+\ldots\right) \in \delta^{r} \Omega[\delta],
$$

so that

$$
\begin{aligned}
& d^{1,0} \omega^{p, q}=0, \\
& d^{0,1} \omega^{p, q}+d^{1,0} \omega_{1}^{p-1, q+1}=0, \\
& d^{-1,2} \omega_{r-3}^{p-r+3, q-r-3}+d^{0,1} \omega_{r-2}^{p-r+2, q+r-2}+d^{1,0} \omega_{r-1}^{p-r+1, q+r-1}=0,
\end{aligned}
$$

From this it follows that

$$
d\left(\omega^{p, q}+\sum_{i>0} \omega_{i}^{p-i, q+i}\right) \in K_{q+r}
$$

which implies

$$
\omega^{p, q} \in D_{r}^{p, q}
$$

From Theorem 2.8 we know

$$
\text { Image } \pi_{i} d_{i} \pi_{i}=\bigoplus_{p, q} \text { Image }\left.\pi_{i} d_{i} \pi_{i}\right|_{E_{i}^{p, q}}
$$

and

$$
\left.\pi_{i} d_{i} \pi_{i}\right|_{E_{i}^{p, q}} \subset E_{i}^{p-i+1, q-i} .
$$

For $\omega^{p, q} \in$ Image $\pi_{l} d_{l} \pi_{i} \cap \Omega^{p, q}$ there is a

$$
v \in E_{i}^{p+i-1, q-i} .
$$

with

$$
\omega^{p, q}=\lim _{\delta \rightarrow 0} \delta^{-i} d_{\delta} \Phi^{\prime}(v)
$$

where

$$
\Phi^{\prime}(v)=v+\delta v_{1}+\delta^{2} v_{2}+\ldots
$$

for some $v_{1}, v_{2}, \ldots$.

Then

$$
\begin{gathered}
d_{\delta}\left[v^{p+l-1, q-i}+\delta v_{1}^{p+l-2, q-i+1}+\delta^{2} v_{2} v^{p+i-3, q-i+2}+\ldots\right] \\
=\delta^{i} \omega^{p, q}+\delta^{l+1} \omega_{1}^{p-1 m q+1}+\delta^{i+2} \omega_{2}^{p-2, q+2}
\end{gathered}
$$

for some

$$
\omega_{i}^{p-i, q+i} \in \Omega^{p-i, q+l} .
$$

Thus

$$
d_{\delta}\left[\omega^{p, q}+\sum_{l>0} \delta^{i} \omega_{i}^{p-i, q+i}\right]=0
$$

or, setting $\delta=1$

$$
d\left\lceil\omega^{p, q}+\sum_{i n} \omega_{i}^{p-i, q+i}\right\rceil=0 .
$$


From (4.10) it follows that

$$
\omega^{p, q} \in D_{\infty}^{p, q} \subseteq D_{r}^{p, q}
$$

which implies

$$
\text { Image } \pi_{i} d_{i} \pi_{i} \cap \Omega^{p, q} \subseteq D_{r}^{p, q} .
$$

The converse of (4.9), as well as part (ii) of this lemma are proved similarly. That is, one simply follows the proof of Lemma 2.7 using the minor modifications demonstrated in our proof of (4.9).

\section{Small Eigenvalues and Eigenspaces}

In this section we assume that $A$ is integrable, and hence defines a foliation of $M$ (so that $d^{2,-1}=d^{2,-1 *}=0$ and $E_{-1}=E_{0}$ ). In addition we make assumptions restricting the local geometry (see hypothesis (H1) and the global geometry (see hypothesis (H2)) of the foliation. We essentially assume that $A$ is a Reimannian foliation with compact leaves, and $g$ is a bundle-like metric (these terms will be defined shortly). There are many examples of such foliations. For example, if

$$
F \hookrightarrow M \rightarrow N
$$

is any fibration of $M$ with compact fiber $F$ then $M$ can be given a metric $g$ such that (5.1) is a Reimannian submersion. Then the foliation of $M$ given by the fibers of (5.1) satisfies the hypotheses. Moreover, if $G$ is a compact Lie group acting on $M$ such that all orbits have the same dimension, and if the metric $G$ is invariant under the action of $G$ (such metrices always exist), then the foliation of $M$ by the orbits of the action satisfy the hypotheses. See [Re] and [Mo] for other examples.

Under these restrictions we prove that for all $p$,

$$
\operatorname{dim} E_{2}^{p}<\infty
$$

This was proven under more general hypotheses in [Sa].

We can then make precise statements about the behavior of the small eigenvalues of $L_{\delta}$, as well as the corresponding eigenspaces, as $\delta \rightarrow 0$. A key role is played by the spaces $\tilde{E}_{k}^{p}$ and the operators $\delta_{k}^{p}$ defined in Sect. 2 .

In particular, we show that the number of eigenvalues which are $\sim \delta^{2 k}$ as $\delta \rightarrow 0$ is precisely $\operatorname{dim} \tilde{E}_{k}^{p}$ (Theorem 5.15), that the corresponding eigenspaces converge to $\tilde{E}_{k}^{p}$ (Theorem 5.17), and that the eigenvalues are asymptotic to the eigenvalues of

$$
\delta^{2 k} \Delta_{k}^{p}: \tilde{E}_{k}^{p} \rightarrow \tilde{E}_{k}^{p}
$$

(Theorem 5.20). Recall that the operator $\Delta_{k}^{p}$ and the spaces $\tilde{E}_{k}^{p}$ in (5.2) are independent of $\delta$, and defined purely in terms of the Taylor series analysis of Sect. 2. That is, loosely speaking, we demonstrate that under the hypotheses $(\mathrm{H} 1)$ and $(\mathrm{H} 2)$, the formal Taylor series analysis of Sect. 2 enables one to conclude precise quantitative statements concerning the asymptotics of the eigenvalues and eigenspaces of $L_{\delta}^{p}$ as $\delta \rightarrow 0$.

We begin this section with a statement of our hypotheses. We first assume (H1) $(M, A, g)$ is a Riemannian foliation with a bundle-like metric. 
That is, we assume $(M, A, g)$ satisfies one of the following equivalent conditions (see [Re] Proposition 4.2):

1) $(M, A, g)$ locally has the structure of a Riemannian submersion.

2) For every vector field $X$ tangent to $A, \mathscr{L}_{X}\left(g_{B}\right)=0$ (where we have written $T M=A+B$, and $g=g_{A}+g_{B}$, and $\mathscr{L}_{X}$ denotes the Lie derivative in the direction $X$ ).

3) The distribution $B$ is totally geodesic, i.e. for every $p \in M, b \in B_{p}$ and every extension of $b$ to a neighborhood of $p$

$$
\left(\nabla_{b} b\right)^{A}(p)=0
$$

(Note that $\left(\nabla_{b} b\right)^{A}(p)$ is independent of the extension.) Let $\nabla_{A}$ denote the map

$$
\nabla_{A}: \Omega^{i, j} \rightarrow T^{*} A \otimes \Omega^{i, j}
$$

given by

$$
\nabla_{A} \omega=\sum_{i=1}^{n} a^{i} \otimes \nabla_{a_{l}}^{0,0} \omega
$$

where $\left\{a_{i}\right\}_{i=1, \ldots, n}$ denotes a basis of $A$ and $\left\{a^{i}\right\}$ is the dual basis of $T^{*} A$. The hypothesis on the local structure of $A$ implies the following:

Lemma 5.1. Assuming the hypothesis $(\mathrm{H} 1)$,

a) The operator $\square^{1,0}=d^{1,0 *}\left(d^{1,0}\right)+\left(d^{1,0}\right)^{*} d^{1,0}$ has the form

$$
\square^{1,0}=\left(\nabla_{A}\right)^{*} \nabla_{A}+C_{1}
$$

where $C_{1}$ is a zero ${ }^{\text {th }}$ order operator.

b) The operator $d^{1,0}\left(d^{0,1}\right)^{*}+\left(d^{0,1}\right)^{*} d^{1,0}$ has the form

$$
d^{1,0}\left(d^{0,1}\right)^{*}+\left(d^{0,1}\right)^{*} d^{1,0}=C_{2} \circ \nabla_{A}+C_{3}
$$

for zero ${ }^{\text {th }}$ order operators $C_{2}$ and $C_{3}$. That is, for a basis $\left\{a_{i}\right\}$ of $A$ there are zero $^{\text {th }}$ order operators $\left\{C_{2, i}\right\}$ and $C_{3}$ such that

$$
d^{1,0}\left(d^{0,1}\right)^{*}+\left(d^{1,0}\right)^{*} d^{1,0}=\sum_{i} C_{2, i} \nabla_{a_{l}}^{0,0}+C_{3} .
$$

The same is true for the operator $\left(d^{1,0}\right)^{*} d^{0,1}+d^{0,1}\left(d^{1,0}\right)^{*}$.

c) The operators $d^{1,0}\left(d^{-1,2}\right)^{*}+\left(d^{-1,2}\right)^{*} d^{1,0}$ and $\left(d^{1,0}\right)^{*} d^{-1,2}+d^{-1,2}\left(d^{1,0}\right)^{*}$ are zero $^{\text {th }}$ order. (This is true independent of $(\mathrm{H} 1)$.)

Proof. At $p \in M$, let $\left\{a_{i}\right\}$ be an orthonormal basis for $A,\left\{b_{j}\right\}$ an orthonormal basis for $B$, and $\left\{a^{j}\right\}$ and $\left\{b^{j}\right\}$ the dual bases. We now extend these bases to orthonormal bases in a neighborhood of $p$. Since $B$ is totally geodesic, we can choose these extensions to be parallel in the $B$ direction so that at $p$,

$$
\nabla_{b_{j}} a_{i}=\nabla_{b_{j}} b_{k}=0 \text { for all } i, j, k \text {. }
$$

We can choose the extension so that, in addition, at $p$

$$
\nabla_{a_{l}}^{0,0} a_{k}=\nabla_{a_{l}}^{0,0} b_{j}=0 \text { for all } i, j, k \text {. }
$$


Then

$$
d^{1,0}=\sum_{i} a^{i} \nabla_{a_{l}}^{0,0}+\sum_{j} b^{j} \nabla_{b_{j}}^{1,-1} .
$$

We observe that at $p \nabla_{b_{j}}^{1,-1}=0$. Since

$$
\left(d^{1,0}\right)^{*}=-\sum \nabla_{a_{k}}^{0,0} i_{a_{k}}+C_{1}
$$

where $C_{1}$ is a zero ${ }^{\text {th }}$ order operator which vanishes at $p$, we have at $p$,

$$
\begin{aligned}
d^{1,0}\left(d^{1,0}\right)^{*}+\left(d^{1,0}\right)^{*} d^{1,0}= & -\sum_{i, k} a^{i} \nabla_{a_{l}}^{0,0} \nabla_{a_{k}}^{0,0} i_{a_{k}}+\nabla_{a_{k}}^{0,0} i_{a_{k}} a^{i} \nabla_{a_{l}}^{0,0} \\
& +\left(\text { zero }{ }^{\text {th }} \text { order term }\right) \\
= & \left(\text { using } \nabla_{a_{l}}^{0,0} a_{j}=0 \text { for all } i, j\right) \\
& -\sum_{i, k} a^{i} a_{k} \nabla_{a_{l}}^{0,0} \nabla_{a_{k}}^{0,0}+i_{a_{k}} a^{i} \nabla_{a_{k}}^{0,0} \nabla_{a_{l}}^{0,0}+(\text { z.o.t. }) \\
= & -\sum_{i, k}\left(a^{i} i_{a_{k}}+i_{a_{k}} a^{i}\right) \nabla_{a_{i}}^{0,0} \nabla_{a_{k}}^{0,0} \\
& \left.-\sum_{i, k} i_{a_{k}} a^{i}\left(\nabla_{a_{k}}^{0,0} \nabla_{a_{i}}^{0,0}-\nabla_{a_{i}}^{0,0} \nabla_{a_{k}}^{0,0}\right)+\text { z.o.t. }\right) .
\end{aligned}
$$

Since $a^{i} i_{a_{k}}+i_{a_{k}} a^{i}=\delta_{i k}$, the first term in (5.3) is, at $p$

$$
-\sum_{i} \nabla_{a_{i}}^{0,0} \nabla_{a_{i}}^{0,0}=\nabla_{A}^{*} \nabla_{A}
$$

Since

$$
\nabla_{a_{k}} \nabla_{a_{i}}-\nabla_{a_{l}} \nabla_{a_{k}}=\nabla_{\left[a_{k}, a_{l}\right]}+R\left(a_{k}, a_{i}\right)
$$

where $R\left(a_{k}, a_{i}\right)$ is a zero ${ }^{\text {th }}$ order curvature operator, we have

$$
\begin{aligned}
\nabla_{a_{k}}^{0,0} \nabla_{a_{l}}^{0,0} & -\nabla_{a_{l}}^{0,0} \nabla_{a_{k}}^{0,0}=R\left(a_{i}, a_{k}\right)^{0,0}+\nabla_{\left[a_{k}, a_{l}\right]}^{0,0} \\
& -\left(\nabla_{a_{k}}^{1,-1} \nabla_{a_{l}}^{-1,1}+\nabla_{a_{k}}^{-1,1} \nabla_{a_{i}}^{1,-1}-\nabla_{a_{l}}^{1,-1} \nabla_{a_{k}}^{-1,1}-\nabla_{a_{l}}^{-1,1} \nabla_{a_{k}}^{-1,-1}\right) .
\end{aligned}
$$

For every $v, \nabla_{v}^{a, b}$ is $0^{\text {th }}$ order if $(a, b) \neq(0,0)$. Furthermore, $\left[a_{k}, a_{i}\right] \subset A$ so

$$
\left[a_{k}, a_{i}\right]=\nabla_{a_{k}}^{0,0} a_{i}-\nabla_{a_{i}}^{0,0}=0 \quad \text { at } p \text {. }
$$

Thus, at $p$

$$
\square^{1,0}=\nabla_{A}^{*} \nabla_{A}+\text { zero }^{\text {th }} \text { order terms. }
$$

Since $p$ is arbitrary, this proves (a).

Parts (b) and (c) are proved similarly.

Corollary 5.2. There are constants $c_{1}$ and $c_{2}$ so that for all $\omega \in \Omega^{*}$,

$$
\begin{aligned}
\left|\left(d^{1,0}\left(d^{0,1}\right)^{*}+\left(d^{0,1}\right)^{*} d^{1,0}\right) \omega\right| & \leqq c_{1}\left(\left|\nabla_{A} \omega\right|+|\omega|\right) \\
& \leqq c_{2}\left(\left\langle\square^{1,0} \omega, \omega\right\rangle^{\frac{1}{2}}+|\omega|\right) .
\end{aligned}
$$

The assumption ( $\mathrm{H} 1)$ is a restriction on the local geometry of $(M, A, g)$. Our second, and last, assumption restricts the global geometry. 
(H2) We assume that the positive spectrum of the operator $\square^{1,0}=d^{1,0}\left(d^{1,0}\right)^{*}+$ $\left(d^{1,0}\right)^{*} d^{1,0}$ is bounded away from 0 by a positive constant.

That is, we assume there is a $c>0$ such that $\omega \in\left(\operatorname{ker} \square^{1,0}\right)^{\perp}$ implies

$$
\left\langle\square^{1,0} \omega, \omega\right\rangle \geqq c|\omega|^{2} .
$$

Lemma 5.3. If $(M, A, g)$ satisfies $(\mathrm{H} 1)$ and, in addition, all leaves of $A$ are compact, then $(M, A, g)$ satisfies $(\mathrm{H} 2)$.

Proof. First suppose $A$ consists of the fibers of a Riemannian submersion $\pi: M \rightarrow N$ with compact fibers. Then $\omega \in \Omega^{l, j}(M, V)$ can be considered as a $j$-form on $N$ with values in the infinite-dimensional bundle

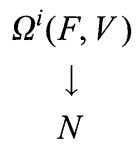

(where $\Omega^{i}(A, V)$ denotes the bundle whose fiber at $x$ is $\Omega^{i}\left(\pi^{-1}(x), V_{\pi^{-1}(x)}\right)$ ) ) For $\omega=\alpha \otimes \beta, \alpha \in \Omega^{i}(A, V)$ and $\beta \in \Omega^{J}(N)$, (or, more precisely, $\omega=\alpha \wedge \pi^{*} \beta$ ) we have

$$
\begin{gathered}
d^{1,0} \omega=\left(d_{A} \alpha\right) \otimes \beta, \\
\left(d^{1,0}\right)^{*} \omega=\left(d_{A}^{*} \alpha\right) \otimes \beta, \\
\square^{1,0} \omega=\left(\square_{A} \alpha\right) \otimes \beta,
\end{gathered}
$$

where $d_{A}, d_{A}^{*}$ and $\square_{A}$ are the differential, codifferential and Laplacian, resp., as an element of $\Omega^{l}\left(\pi^{-1}(x), V_{\pi^{-1}(x)}\right)$. Thus

$$
\operatorname{ker} \square^{1,0}=\Gamma\left(N, \Lambda^{*} T^{*} N \otimes \mathscr{H}^{*}(A, V)\right),
$$

where $\mathscr{H}^{*}(A, V)$ denotes the vector bundle over $N$ whose fiber at $x$ are the harmonic forms on $\pi^{-1}(x)$ with values in $V$. Moreover

$$
\begin{aligned}
& \inf \left\{\lambda \in \operatorname{spec} \square^{1,0} \mid \lambda>0\right\} \\
& \quad=\inf _{x \in N}\left[\inf \left\{\lambda \in \operatorname{spec} \square_{N}: \Omega^{*}\left(\pi^{-1}(x), V_{\pi^{-1}(x)}\right) \supset \mid \lambda>0\right\}\right] .
\end{aligned}
$$

The spectrum of $\square_{A}$ varies continuously over $N$, and the multiplicity of 0 is constant. Thus the smallest positive eigenvalue is a continuous function on $N$ and therefore achieves a positive minimum. This implies the infimum in (5.4) is positive, which is precisely $(\mathrm{H} 2)$.

More generally, suppose $A$ is any foliation with compact leaves satisfying (H1). Then, by Proposition 3.7 of [Mo], the leaf space $N=M / A$ has the structure of a compact Satake $V$-manifold (see [Mo] for a precise definition). We will show that the infimum in (5.4) is positive by showing that the function

$$
\lambda(x)=\inf \left\{\lambda \in \operatorname{spec} \square_{A}: \Omega^{*}\left(\pi^{-1}(x), V\right) \supset \mid \lambda>0\right\}
$$

is locally bounded away from 0 (from which $(\mathrm{H} 2)$ follows by the compactness of $N) . M$ is a locally trivial fibration over the dense set $U$ of non-singular points in $N$, and at any $x \in U$ the local boundedness of $\lambda(x)$ follows as before. 
If $x$ is a singular point of $N$, then all nearby leaves cover $\pi^{-1}(x)$, with the degree of the covers bounded above by some $k$. Suppose $\left\{\mathscr{C}_{1}, \ldots, \mathscr{C}_{k}\right\}$ are all the Riemannian covers of $\pi^{-1}(x)$ with degree $\leqq k$, and

$$
\lambda\left(\mathscr{C}_{i}\right)=\inf \left\{\lambda \in \operatorname{spec} \square: \Omega^{*}(\mathscr{C}, V) \supset \mid \lambda>0\right\},
$$

where $V$ in (5.6) denotes the pull-back to $\mathscr{C}_{i}$ of the bundle $V$ over $\pi^{-1}(x)$.

There is a neighborhood of $x$ which can be stratified by sets $\sum_{i}$ such that $\left\{\pi^{-1}\left(x^{\prime}\right) \mid x^{\prime} \in \sum_{i}\right\}$ are all diffeomorphic and as $x^{\prime} \in \sum_{i}$ approaches $\left.x, \pi^{-1}\right)\left(x^{\prime}\right)$ approaches some $\mathscr{C}_{j}$. Thus, as $x^{\prime} \in \sum_{i}$ approaches $x, \lambda\left(x^{\prime}\right)$ approaches $\lambda\left(\mathscr{C}_{j}\right)>0$, which proves $\lambda$ is locally bounded away from 0 .

From now on, we assume hypotheses (H1) and (H2). The significance of these assumptions is hinted at in the following lemma.

Lemma 5.4. There is a $c>0$ such that for all $\delta$ small enough,

$$
L_{\delta} \geqq \frac{1}{2} \square^{1,0}+\delta^{2}\left(\square^{0,1}-C\right) \geqq \delta^{2}\left(\square^{1,0}+\square^{0,1}-C\right),
$$

where $\square^{a, b}=d^{a, b}\left(d^{a, b}\right)^{*}+\left(d^{a, b}\right)^{*} d^{a, b}$.

Proof.

$$
\begin{aligned}
L_{\delta}= & \square^{1,0}+\delta^{2} \square^{0,1}+\delta^{4} \square^{-1,2}+\delta\left(d^{1,0}\left(d^{0,1}\right)^{*}\right. \\
& \left.+\left(d^{0,1}\right)^{*} d^{1,0}+\left(d^{1,0}\right)^{*} d^{0,1}+d^{0,1}\left(d^{1,0}\right)^{*}\right) \\
& +\delta^{2}\left(d^{1,0}\left(d^{-1,2}\right)^{*}+\left(d^{-1,2}\right)^{*} d^{1,0}+\left(d^{1,0}\right)^{*} d^{-1,2}+d^{-1,2}\left(d^{1,0}\right)^{*}\right) \\
& +\delta^{3}\left(d^{0,1}\left(d^{-1,2}\right)^{*}+\left(d^{-1,2}\right)^{*} d^{0,1}+\left(d^{0,1}\right)^{*} d^{-1,2}+d^{1,2}\left(d^{0,1}\right)^{*}\right) .
\end{aligned}
$$

To simplify notation we will write

$$
L_{\delta}=\square^{1,0}+\delta^{2} \square^{0,1}+\delta^{4} \square^{-1,2}+\delta K_{1}+\delta^{2} K_{2}+\delta^{3} K_{3} .
$$

We observe that $\square^{-1,2}$ is positive, and from Lemma 5.1 (c), $K_{2}$ is a zero ${ }^{\text {th }}$ order operator, so for some $c_{1}$,

$$
L_{\delta} \geqq \square^{1,0}+\delta^{2} \square^{0,1}+\delta K_{1}+\delta^{3} K_{3}-c_{1} \delta^{2} .
$$

We now observe that for any $\omega$

$$
\left\langle\delta^{3} K_{3} \omega, \omega\right\rangle=2\left\langle\delta^{3 / 2} d^{0,1} \omega, \delta^{3 / 2} d^{-1,2} \omega\right\rangle+2\left\langle\delta^{3 / 2}\left(d^{0,1}\right)^{*} \omega, \delta^{3 / 2}\left(d^{-1,2}\right)^{*} \omega\right\rangle,
$$

so that

$$
\left|\left\langle\delta^{3} K_{3} \omega, \omega\right\rangle\right| \leqq \delta^{3}\left\langle\square^{0,1} \omega, \omega\right\rangle+\delta^{3}\left\langle\square^{-1,2} \omega, \omega\right\rangle .
$$

Thus, for $\delta \leqq \frac{1}{2}$ there is a $c_{2}$ so that

$$
L_{\delta} \geqq \square^{1,0}+\frac{1}{2} \delta^{2} \square^{0,1}+\delta K_{1}-c_{2} \delta^{2} .
$$

Lastly, we write

$$
\delta\left\langle K_{1} \omega, \omega\right\rangle=\delta\left(\left\langle K_{1} \tilde{\pi}_{0} \omega, \tilde{\pi}_{0} \omega\right\rangle+2\left\langle K_{1} \pi_{1} \omega, \tilde{\pi}_{0} \omega\right\rangle+\left\langle K_{1} \pi_{1} \omega, \pi_{1} \omega\right\rangle\right) .
$$


We note that

$$
\left\langle K_{1} \pi_{1} \omega, \pi_{1} \omega\right\rangle=0
$$

and, from Corollary 5.2, there is a $c_{3}$ such that

$$
\left|K_{1} \pi_{1} \omega\right| \leqq c_{3}\left|\pi_{1} \omega\right| \text {. }
$$

Thus, for any $\varepsilon>0$,

$$
\begin{aligned}
\left|\delta\left\langle K_{1} \pi_{1} \omega, \tilde{\pi}_{0} \omega\right\rangle\right| & \leqq 2 \delta c_{3}\left|\pi_{1} \omega\right|\left|\tilde{\pi}_{0} \omega\right|=2\left|\varepsilon \tilde{\pi}_{0} \omega\right|\left|\frac{\delta c_{3}}{\varepsilon} \pi_{1} \omega\right| \\
& \leqq \varepsilon^{2}\left|\tilde{\pi}_{0} \omega\right|^{2}+\delta^{2}\left(\frac{c_{3}}{\varepsilon^{2}}\left|\pi_{1} \omega\right|^{2}\right) .
\end{aligned}
$$

From $(\mathrm{H} 2)$ we can choose $\varepsilon$ small enough so that for all $\omega$,

$$
\frac{1}{4}\left\langle\square^{1,0} \omega, \omega\right\rangle \geqq \varepsilon^{2}\left|\pi_{0} \omega\right|^{2}
$$

For such $\varepsilon$

$$
2\left\langle\delta K_{1} \pi_{1} \omega, \tilde{\pi}_{0} \omega\right\rangle \geqq-\left(\frac{1}{4}\left\langle\square^{1,0} \omega, \omega\right\rangle+\delta^{2}\left(\frac{c_{3}}{\varepsilon^{2}}\right)|\omega|^{2}\right) .
$$

From Corollary 5.2 there is a $c_{4}$ such that

$$
\left|\left\langle\delta K_{1} \tilde{\pi}_{0} \omega, \tilde{\pi}_{0} \omega\right\rangle\right| \leqq \delta c_{4}\left(\left\langle\square^{1,0} \tilde{\pi}_{0} \omega, \tilde{\pi}_{0} \omega\right\rangle^{1 / 2}\left|\tilde{\pi}_{0} \omega\right|+\left|\tilde{\pi}_{0} \omega\right|^{2}\right) .
$$

We have from (H2),

$$
\delta c_{4}\left|\pi_{0} \omega\right|^{2} \leqq \delta c_{5}\left\langle\square^{1,0} \omega, \omega\right\rangle
$$

so for $\delta$ small enough

$$
\delta c_{4}\left|\pi_{0} \omega\right|^{2} \leqq \frac{1}{8}\left\langle\square^{1,0} \omega, \omega\right\rangle
$$

Moreover

$$
\begin{aligned}
& \delta c_{4}\left\langle\square^{1,0} \tilde{\pi}_{0} \omega, \tilde{\pi}_{0} \omega\right\rangle^{1 / 2}|\tilde{\pi} \omega|=2\left(\frac{1}{\sqrt{8}}\left\langle\square^{1,0} \tilde{\pi}_{0} \omega, \tilde{\pi}_{0} \omega\right\rangle^{1 / 2}\right)\left(\frac{\delta c_{4} \sqrt{8}}{2}\left|\pi_{0} \omega\right|^{2}\right) \\
& \left.\left.\leqq \frac{1}{8}\left\langle\square^{1,0} \tilde{\pi}_{0} \omega, \tilde{\pi} \omega\right\rangle+\delta^{2}\right) 2 c_{4}^{2}\right)\left|\pi_{0} \omega\right|^{2} \leqq \frac{1}{8}\left\langle\square^{1,0} \omega, \omega\right\rangle+\delta^{2}\left(2 c_{4}^{2}\right)|\omega|^{2} .
\end{aligned}
$$

Combining (5.7)-(5.13) yields the theorem.

We observe that $\square^{1,0}+\square^{0,1}$ is an elliptic $2^{\text {nd }}$ order operator with positive symbol. Thus, for any $k \in \mathbf{R}$, only finitely many eigenvalues of $\square^{1,0}+\square^{0,1}-C$ are less than $k$.

Combining this observation with Lemma 5.4 we see that at most finitely many eigenvalues of $L_{\delta}^{p}$ can be in $0\left(\delta^{4}\right)$. Therefore, from (1.8) we learn

Corollary 5.5. For all $p$

$$
\operatorname{dim} E_{2}^{p}<\infty
$$

Before leaving this topic, we present an implication of (H1) and (H2) which will play an important role in the analysis in this section. 
Lemma 5.6. There is a $c>0$ such that

$$
\left|\tilde{\pi}_{0} d^{0,1} \pi_{1}\right|,\left|\tilde{\pi}_{0}\left(d^{0,1}\right)^{*} \pi_{1}\right|,\left|\pi_{1} d^{0,1} \tilde{\pi}_{0}\right|,\left|\pi_{1}\left(d^{0,1}\right)^{*} \tilde{\pi}_{0}\right|
$$

are all bounded above by $c$.

Proof. We prove the lemma for $\tilde{\pi}_{0} d^{0,1} \pi_{1}$. The bound for the other operators follows similarly. Suppose $\omega \in \Omega^{p}$ satisfies $\omega \in E_{1}=\mathrm{Kernel}^{1,0}=\mathrm{Kernel}^{1,0} \cap$ $\operatorname{Kernel}\left(d^{1,0}\right)^{*}$. Write $d^{0,1} \omega=\alpha+\beta$ with

$$
\begin{aligned}
& \alpha \in E_{1}, \\
& \beta \in \tilde{E}_{0}=E_{1}^{\perp}=\left(\text { Kernel } \square^{1,0}\right)^{\perp}=\operatorname{Image} d^{1,0} \oplus \operatorname{Image}\left(d^{1,0}\right)^{*} .
\end{aligned}
$$

Since

$$
d^{1,0}\left(d^{0,1} \omega\right)=-d^{0,1}\left(d^{1,0} \omega\right)=0
$$

and

$$
d^{1,0} \alpha=0
$$

we must have

$$
\beta \in \operatorname{Kernel} d^{1,0} \text {. }
$$

Then, from Corollary 5.2,

$$
\begin{aligned}
c_{1}|\omega| & \geqq\left|\left(d^{0,1}\left(d^{1,0}\right)^{*}+\left(d^{1,0}\right)^{*} d^{0,1}\right) \omega\right| \\
& =\left|\left(d^{1,0}\right)^{*} d^{0,1} \omega\right|=\left|\left(d^{1,0}\right)^{*} \beta\right| \\
& =\left|\left(d^{1,0}+\left(d^{1,0}\right)^{*}\right) \beta\right| \geqq c_{2}|\beta|
\end{aligned}
$$

(from $(\mathrm{H} 2))$

$$
=c_{2}\left|\tilde{\pi}_{0} d^{0,1} \omega\right| \text {. }
$$

Thus, we have proven Lemma 4.6 with $c=\frac{c_{1}}{c_{2}}$.

We now commence our study of the small eigenvalues of $L_{\delta}$. We begin by showing that the number of eigenvalues which are in $o\left(\delta^{2}\right)$ as $\delta \rightarrow 0$ is precisely $\operatorname{dim} E_{2}^{p}$.

The first step is to show that the corresponding eigenspaces converge to $E_{2}^{p}$ and $\delta \rightarrow 0$.

Lemma 5.7. Given any $c_{1}>0$ there is a $c_{2}>0$ such that:

If $\delta_{i}, i=1,2,3, \ldots$, is any sequence with $\delta_{i} \rightarrow 0$, and $\omega_{i}$ any sequence of $p$ forms with $\left|\omega_{i}\right|=1$ and

$$
\left\langle L_{\delta_{i}} \omega_{i}, \omega_{i}\right\rangle \leqq c_{1} \delta^{2}
$$

then

$$
\left|\tilde{\pi}_{0} \omega_{l}\right| \leqq c_{2} \delta_{l}
$$

Proof. This follows directly from Lemma 5.4 and (H2) which imply

$$
c_{1} \delta^{2} \geqq\left\langle L_{\delta_{i}} \omega_{i}, \omega_{i}\right\rangle \geqq\left\langle\square^{1,0} \omega, \omega\right\rangle-c_{3} \delta^{2} \geqq c_{4}\left|\tilde{\pi}_{0} \omega\right|^{2}-c_{3} \delta^{2} .
$$

Theorem 5.8. If $\delta_{i}, i=1,2,3, \ldots$, is any sequence with $\delta_{i} \rightarrow 0$, and $\omega_{i}$ any sequence of p-forms satisfying $\left|\omega_{i}\right|=1$ and 


$$
\left\langle L_{\delta_{t}} \omega_{i}, \omega_{i}\right\rangle \in o\left(\delta^{2}\right),
$$

then a subsequence of the $\omega_{i}$ 's converges (strongly in $L^{2}$ ) to an element $\omega \in E_{2}^{p}$. Proof. Write $\omega_{l}=\alpha_{l}+\beta_{l}$ with

$$
\alpha_{\imath} \in \tilde{E}_{0}^{p}=\left(\operatorname{ker} \square^{1,0}\right)^{\perp}
$$

and

$$
\beta_{i} \in E_{1}^{p}=\operatorname{ker} \square^{1,0} .
$$

From Lemma 5.7 it follows that there is a $c>0$ with

$$
\left|\alpha_{l}\right|<c \delta_{i}
$$

so that

$$
\left|\beta_{l}\right|=1+O\left(\delta_{i}\right) .
$$

The lemma follows once we show that a subsequence of the $\beta_{i}$ 's converges to an element in $E_{2}^{p}$.

(i) First we show that the $\beta_{i}$ 's are bounded in $H_{1}$. By definition we have

$$
\left|d^{1,0} \beta_{i}\right|=\left|\left(d^{1,0}\right)^{*} \beta\right|=0,
$$

so it is enough to show that for some $k>0$,

$$
\left|d^{0,1} \beta_{l}\right|<k, \quad\left|\left(d^{0,1}\right)^{*} \beta_{l}\right|<k .
$$

Now $\left|d_{\delta} \omega\right|^{2} \in o\left(\delta^{2}\right)$ implies

$$
\left|d^{1,0} \omega+\delta d^{0,1} \omega+\delta^{2} d^{-1,2} \omega\right| \in o\left(\delta^{2}\right) \Rightarrow \pi_{1}\left(d^{0,1} \omega+\delta d^{-1,2} \omega\right) \rightarrow 0 \Rightarrow \pi_{1} d^{0,1} \omega \rightarrow 0 .
$$

But

$$
\pi_{1} d^{0,1} \omega=\pi_{1} d^{0,1}(\alpha+\beta)=\left(\pi_{1} d^{0,1} \tilde{\pi}_{0}\right) \alpha+\pi_{1} d^{0,1} \beta .
$$

From Lemma 5.6

$$
\left|\pi_{1} d^{0,1} \tilde{\pi}_{0} \alpha\right| \leqq c|\alpha| \in O(\delta)
$$

Thus

$$
\left|\pi_{1} d^{0,1} \beta\right| \rightarrow 0
$$

On the other hand

$$
\left|\tilde{\pi}_{0} d^{0,1} \beta\right|=\left|\tilde{\pi}_{0} d^{0,1} \pi_{1} \beta\right| \leqq c|\beta| \leqq c|\omega|=c .
$$

Together, (5.14) and (5.15) imply

$$
\left|d^{0,1} \beta\right|<k
$$

Similarly, one can prove

$$
\left|\left(d^{0,1}\right)^{*} \beta\right|<k .
$$

Thus the $\beta_{i}$ 's are bounded in $H_{1}$ and hence a subsequence of the $\beta_{l}$ 's converges weakly in $H_{1}\left(\Rightarrow\right.$ strongly in $L^{2}$ ) to some $\beta \in H_{1}$.

(ii) $\beta \in E_{1}^{p}$ : Since $\beta_{i} \rightarrow \beta$ strongly in $L^{2}$, (2) implies $|\beta|=1$. Now

$$
0=\tilde{\pi}_{0} \beta_{i} \rightarrow \tilde{\pi}_{0} \beta
$$

implies $\tilde{\pi}_{0} \beta=0$ so $\beta \in E_{1}^{p}$. 

Thus

(iii) $\beta \in E_{2}^{p}$ : Since $\beta_{i} \rightarrow \beta$ weakly in $H_{1}$ we have $d^{0,1} \beta_{i} \rightarrow d^{0,1} \beta$ weakly in $L^{2}$.

$$
\pi_{1} d^{0,1} \beta_{i} \rightarrow \pi_{1} d^{0,1} \beta
$$

weakly in $L^{2}$. By (5.14) $\left|\pi_{1} d^{0,1} \beta_{i}\right| \rightarrow 0$. This implies $\pi_{1} d^{0,1} \beta_{i} \rightarrow 0$ strongly in $L^{2}$. Therefore (by the uniqueness of weak limits)

$$
\pi_{1} d^{0,1} \beta=0 \text {. }
$$

Similarly

$$
\pi_{1}\left(d^{0,1}\right)^{*} \beta=0 .
$$

Together, (5.16) and (5.17) imply $\beta \in E_{2}^{p}$.

\section{Corollary 5.9.}

$$
\#\left\{\lambda_{i}^{p} \in \operatorname{spec}\left(L_{\delta}^{p}\right) \mid \liminf _{\delta \rightarrow 0} \delta^{-2} \lambda_{i}^{p}=0\right\}=E_{2}^{p} .
$$

Proof. The inequality $\geqq$ follows from (1.8). If there were a strict inequality, then we could find $\delta_{i} \rightarrow 0$ and $\omega_{i} \in \Omega^{p}$ with

$$
\left|\omega_{i}\right|=1,\left\langle L_{\delta_{l}} \omega_{i}, \omega_{i}\right\rangle \in o\left(\delta^{2}\right)
$$

and $\left\langle\omega_{i}, \omega\right\rangle=0$ for every $\omega \in E_{2}^{p}$, but this contradicts Theorem 5.8.

We saw in (1.8) that

$$
\#\left\{\lambda_{i}^{p} \in O\left(\delta^{4}\right)\right\} \geqq \operatorname{dim} E_{2}^{p} .
$$

Combining this with Corollary 5.9 we have

Corollary 5.10. There are constants $c_{1}, c_{2}>0$ such that for every $i$ either

$$
\lambda_{i}^{p} \geqq c_{1} \delta^{2} \quad \text { for all } \delta
$$

or

$$
\lambda_{i}^{p} \leqq c_{2} \delta^{4} \quad \text { for all } \delta
$$

Our next goal is to prove analogous statements about eigenvalues which are $O\left(\delta^{2 k}\right)$.

To analyze the small eigenvalues, it is convenient to modify the $\tilde{E}_{k}$ spaces by using the extension map $\Phi^{\prime}$ defined in Sect. 2.

Recall that for each $k, \Phi^{\prime}$ is a linear map

$$
\Phi^{\prime}: \tilde{E}_{k}^{p} \rightarrow \Omega^{p}[\delta]
$$

such that for $v \in \tilde{E}_{k}^{p}$

$$
\begin{aligned}
& d_{\delta} \Phi^{\prime}(v) \in \delta^{k} \tilde{E}_{k}^{p+1}+\delta^{k+1} \Omega^{p+1}[\delta] \\
& d_{\delta}^{*} \Phi^{\prime}(v) \in \delta^{k} \tilde{E}_{k}^{p-1}+\delta^{k+1} \Omega^{p-1}[\delta]
\end{aligned}
$$

For each $v \in E_{N}^{p}$, there is a formal power series

$$
v_{\delta}=v+\delta v_{1}+\delta^{2} v_{2}+\ldots
$$


such that the map $v \mapsto v_{\delta}$ is linear, and, formally,

$$
d_{\delta} v_{\delta}=d_{\delta}^{*} v_{\delta}=0
$$

Define, for $v \in E_{N}^{p}$

$$
\Phi^{\prime}(v)=v+\delta v_{1}+\delta^{2} v_{2}+\cdots+\delta^{N+2} v_{N+2}
$$

(where the $v_{i}$ 's are as in $(5.18)$ ), so that

$$
\begin{aligned}
& d_{\delta} \Phi^{\prime}(v) \in \delta^{N+3} \Omega^{p+1}[\delta], \\
& d_{\delta}^{*} \Phi^{\prime}(v) \in \delta^{N+3} \Omega^{p-1}[\delta]
\end{aligned}
$$

Now extend $\Phi^{\prime}$ linearly to a map

$$
\Phi^{\prime}: \Omega^{p} \rightarrow \Omega^{p}[\delta] .
$$

For every $\delta \in[0,1]$ we can evaluate $\Phi^{\prime}(v)$ at $\delta$ to get a map

$$
\left.\Phi^{\prime}\right|_{\delta}: \Omega^{p} \rightarrow \Omega^{p}
$$

Let $E_{k, \delta}^{p}$ be the image of $E_{k}^{p}$ under this map, and let $\pi_{k, \delta}^{\perp}$ denote the orthogonal projection onto the complement of $E_{k, \delta}^{p}$. Define $\tilde{E}_{k, \delta}^{p}$ to be the orthogonal complement of $E_{k+1, \delta}^{p}$ in $E_{k, \delta}^{p}$, i.e.

$$
\tilde{E}_{k, \delta}^{p}=\pi_{k+1, \delta}^{\perp} E_{k, \delta}^{p}
$$

and $\tilde{\pi}_{k, \delta}$ the orthogonal projection onto $\widetilde{E}_{k, \delta}^{p}$.

Suppose $v_{1}, \ldots, v_{r}$ is the basis of $E_{k+1}^{p}$, so that $\left.\Phi^{\prime}\left(v_{1}\right)\right|_{\delta}, \ldots,\left.\Phi^{\prime}\left(v_{r}\right)\right|_{\delta}$ span $E_{k+1, \delta}^{p}$. If $v \in \tilde{E}_{k}^{p}$, the

$$
\tilde{\pi}_{k, \delta} \Phi^{\prime}(v)=\Phi^{\prime}(v)-\sum_{j=1}^{r}\left\langle\Phi^{\prime}(v), \Phi^{\prime}\left(v_{j}\right)\right\rangle \Phi^{\prime}\left(v_{j}\right) .
$$

Each $\left\langle\Phi^{\prime}(v), \Phi^{\prime}\left(v_{j}\right)\right\rangle$ is in $O(\delta)$, since $\Phi^{\prime}(v)=v+O(\delta), \Phi^{\prime}\left(v_{j}\right)=v_{j}+O(\delta)$ and $\left\langle v, v_{j}\right\rangle=0$. Therefore,

$$
\begin{aligned}
\tilde{\pi}_{k, \delta} \Phi^{\prime}(v) & =v+O(\delta) \\
d_{\delta} \tilde{\pi}_{k, \delta} \Phi^{\prime}(v) & =d^{k} d_{k}^{\prime} v+O\left(d^{k+1}\right)
\end{aligned}
$$

(since $d_{\delta} \Phi^{\prime}\left(v_{j}\right) \in O\left(\delta^{k+1}\right)$ for all $\left.j\right)$, and $d_{\delta}^{*} \tilde{\pi}_{k, \delta} \Phi^{\prime}(v)=\delta^{k}\left(d_{k}^{\prime}\right)^{*} v+O\left(\delta^{k+1}\right.$. Furthermore, we have that for every $\delta \in[0,1]$,

$$
\tilde{E}_{k, \delta}^{p}=\left\{\tilde{\pi}_{k, \delta} \Phi^{\prime}(v) \mid v \in \tilde{E}_{p}^{k}\right\}
$$

Suppose $k>0$, and $v_{\delta} \in \tilde{E}_{k, \delta}^{p}$ for $\delta \in[0,1]$. Thus

$$
v_{\delta}=\tilde{\pi}_{k, \delta} \Phi^{\prime}\left(\tilde{v}_{\delta}\right)
$$

for some 1-parameter family of elements

$$
\tilde{v}_{\delta} \in \tilde{E}_{k}^{p} .
$$


Then

$$
\left\langle L_{\delta} v_{\delta}, v_{\delta}\right\rangle=\delta^{2 k}\left\langle\Delta_{k} \tilde{v}_{\delta}, \tilde{v}_{\delta}\right\rangle+O\left(\delta^{2 k+1}\right)
$$

(where $\left.\Delta_{k}=d_{k}^{\prime}\left(d_{k}^{\prime}\right)^{*}+\left(d_{k}^{\prime}\right)^{*} d_{k}^{\prime}\right)$.

From Theorem 2.5 (b)

$$
\Delta_{k}: \tilde{E}_{k} \rightarrow \tilde{E}_{k}
$$

in invertible. Since $\tilde{E}_{k}$ is finite dimensional for $k>1$, there are positive constants $c_{1}$ and $c_{2}$ such that for all $v \in \tilde{E}_{k}$,

$$
c_{1} \delta^{2 k}|v|^{2}<\left\langle\Delta_{k} v, v\right\rangle<c_{2} \delta^{2 k}|v|^{2} .
$$

Combining (5.19) and (5.20) we find

Lemma 5.11. For every $k>1$ there are constants $c_{1}, c_{2}>0$ such that for all $v_{\delta} \in \tilde{E}_{k, \delta}$,

$$
c_{1} \delta^{2 k}\left|v_{\delta}\right|^{2}<\left\langle L_{\delta} v_{\delta}, v_{\delta}\right\rangle<c_{2} \delta^{2 k}\left|v_{\delta}\right|^{2} .
$$

In addition, we know from the definition of $\Phi^{\prime}$ that if $v \in \tilde{E}_{k}$, then

$$
d_{k}^{\prime} v,\left(d_{k}^{\prime}\right)^{*} v \in \tilde{E}_{k} .
$$

Thus, if follows for $v_{1} \in \tilde{E}_{k}, v_{2} \in \tilde{E}_{l}, k \neq l$, we have that

$$
\begin{aligned}
& \left\langle d_{\delta} \tilde{\pi}_{k, \delta} \Phi^{\prime}\left(v_{1}\right), d_{\delta} \tilde{\pi}_{l, \delta} \Phi^{\prime}\left(v_{2}\right)\right\rangle \in O\left(\delta^{k+l+1}\right), \\
& \left\langle d_{\delta}^{*} \tilde{\pi}_{k, \delta} \Phi^{\prime}\left(v_{1}\right), d_{\delta}^{*} \tilde{\pi}_{l, \delta} \Phi^{\prime}\left(v_{2}\right)\right\rangle \in O\left(\delta^{k+l+1}\right) .
\end{aligned}
$$

This implies that for any $v$ and $w$,

$$
\left\langle\tilde{\pi}_{l, \delta} L_{\delta} \tilde{\pi}_{k, \delta} v, w\right\rangle \in O\left(\delta^{k+l+1}\right) .
$$

If $k>1$ or $l>1$, then $\tilde{\pi}_{l, \delta} L_{\delta} \tilde{\pi}_{k, \delta}$ has finite rank, so the bound in (5.21) is uniform in $v$ and $w$. That is

Lemma 5.12. There is a $c>0$ such that for all $k$ and $l$ with

$$
\max \{k, l\} \geqq 2 \text {. }
$$

we have

$$
\left|\tilde{\pi}_{l, \delta} L_{\delta} \tilde{\pi}_{k, \delta}\right| \leqq \begin{cases}c \delta^{k+l} & \text { if } k=1 \\ c \delta^{k+l+1} & \text { if } k \neq l\end{cases}
$$

This brings us to the theorem from which our main results will be derived.

Theorem 5.13.

a) There is a $c>0$ such that for $k \geqq 1$ restricted to $E_{k, \delta}^{\perp}$,

$$
\pi_{k, \delta}^{\perp} L_{\delta} \pi_{k, \delta}^{\perp}>c \delta^{2(k-1)} .
$$

b) For every $c_{2}>0$ there is a $c_{2}>0$ such that for all $k \geqq 1, \delta \in(0,1]$ and $v_{\delta} \in E_{k, \delta}^{\perp}$ if $\left|v_{\delta}\right|=1$ and $\left\langle L_{\delta} v_{\delta}, v_{\delta}\right\rangle \leqq c_{1} \delta^{2(k-1)}$ then for all $0 \leqq i \leqq k-1$,

$$
\left|\tilde{\pi}_{i, \delta} v_{\delta}\right| \leqq c_{2} \delta^{k-i-1}
$$


c) There is a $c>0$ such that for every $k \geqq 1$, and every $i, j$ with $0 \leqq i, j \leqq$ $k-1$,

$$
\left|\tilde{\pi}_{i, \delta}\left(\pi_{k, \delta}^{\perp} L_{\delta} \pi_{k, \delta}^{\perp}\right)^{-1} \tilde{\pi}_{j, \delta}\right| \leqq c \delta^{-i-j}
$$

Proof. Parts (a), (b) and (c) are intimately related, and we prove them simultaneously, inductively in $k$

$$
\begin{aligned}
& k=1: \text { Part (a) follows from Lemma } 5.4 \text { and Hypothesis (H2) } \\
& \text { Part (b) is vacuous } \\
& \text { Part (c) follows directly from (a) } \\
& k=2: \text { Part (a) is Theorem } 5.8 \text { ) } \\
& \text { Part (b) is Lemma 5.7. }
\end{aligned}
$$

We now proceed inductively.

Proof of (c). Assume (a) (b) and (c) have been proved for $k-1$, and (a) and (b) have been proved for $k \geqq 2$. The proof of part (c) will be by downward induction on $\max \{i, j\}$.

$\max \{\mathbf{i}, \mathbf{j}\}=\mathbf{k}-1$. Assume $j=k-1$. We will show that for all $i \leqq k-1$ (leaving off the $\delta$ subscripts)

$$
\left|\tilde{\pi}_{i}\left(\pi_{k}^{\perp} L \pi_{k}^{\perp}\right)^{-1} \tilde{\pi}_{k-1}\right| \leqq c \delta^{-(k-1)-i}
$$

Since

$$
\left(\tilde{\pi}_{i}\left(\pi_{k}^{\perp} L \pi_{k}^{\perp}\right)^{-1} \tilde{\pi}_{j}\right)^{*}=\tilde{\pi}_{j}\left(\pi_{k}^{\perp} L \pi_{k}^{\perp}\right)^{-1} \tilde{\pi}_{i}
$$

and taking adjoints preserves norms, this implies the same inequality for $j \leqq i=$ $k-1$.

Suppose $v \in \tilde{E}_{k-1}$ and $|v|=1$, and let

$$
\left(\pi_{k}^{\perp} L \pi_{k}^{\perp}\right)^{-1} v=\omega .
$$

Then, by part (a), $|\omega| \leqq c \delta^{-2(k-1)}$. Let

$$
\tilde{\omega}=c^{-1} \delta^{2(k-1)} \omega
$$

so that $|\tilde{\omega}| \leqq 1$. Then $\tilde{\omega} \in E_{k, \delta}^{\perp}$ and

$$
\langle L \tilde{\omega}, \tilde{\omega}\rangle=\left\langle\left(\pi_{k}^{\perp} L \pi_{k}^{\perp}\right) \tilde{\omega}, \tilde{\omega}\right\rangle=c^{-1} \delta^{2(k-1)}\langle v, \tilde{\omega}\rangle \leqq c^{-1} \delta^{2(k-1)} .
$$

Therefore, by part (b) of this theorem, there is a $\bar{c}>0$ with

$$
\left|\tilde{\pi}_{i} \tilde{\omega}\right| \leqq \bar{c} \delta^{k-1-i}
$$

which implies

$$
\left|\tilde{\pi}_{i}\left(\pi_{k}^{\perp} L \pi_{k}^{\perp}\right) \tilde{\pi}_{k-1} v\right|=\left|\tilde{\pi}_{l} \omega\right| \leqq(c \bar{c}) \delta^{-(k-1)-i},
$$

as desired.

$\mathbf{1} \leqq \max \{\mathbf{i}, \mathbf{j}\}=\mathbf{k}-\mathbf{r}, \mathbf{r}>\mathbf{1}$. We assume (5.16) has been proven for $\max \{i, j\}>$ $k-r$. As above, it is sufficient to assume $i \leqq j=k-r$. Suppose $v \in \widetilde{E}_{j, \delta,}|v|=1$ and write 


$$
\left(\pi_{k}^{\perp} L \pi_{k}^{\perp}\right)^{-1} v=\omega
$$

so that

$$
\pi_{k-r+1}^{\perp} L \pi_{k}^{\perp} \omega=v .
$$

Since $v \in \widetilde{E}_{k-r} \subset E_{k-r+1}^{\perp}$ we have

$$
\pi_{k-r+1}^{\perp} L \pi_{k}^{\perp} \omega=v .
$$

Therefore

$$
\pi_{k-r+1}^{\perp} L \pi_{k-r+1}^{\perp} \omega=v-\sum_{l=k-r+1}^{k-1} \pi_{k-r+1}^{\perp} L \tilde{\pi}_{l} \omega
$$

so for $i \leqq k-r$

$$
\begin{aligned}
\tilde{\pi}_{i} \omega & =\sum_{m=0}^{k-r} \tilde{\pi}_{i}\left(\pi_{k-r+1}^{\perp} L \pi_{k-r+1}^{\perp}\right)^{-1} \tilde{\pi}_{m}\left[v-\sum_{l=k-r+1}^{k-1} \pi_{k-r+1}^{\perp} L \tilde{\pi}_{l} \omega\right] \\
& =\tilde{\pi}_{i}\left(\pi_{k-r+1}^{\perp} L \pi_{k-r+1}^{\perp}\right)^{-1} \tilde{\pi}_{k-r} v-\sum_{m=0 l=k-r+1}^{k-r} \sum_{k-1}^{k-1} \tilde{\pi}_{l}\left(\pi_{k-r+1}^{\perp} L \pi_{k-r+1}^{\perp}\right)^{-1} \tilde{\pi}_{m} L \tilde{\pi}_{m} L \tilde{\pi}_{l} \omega .
\end{aligned}
$$

By induction on part (c),

$$
\left|\tilde{\pi}_{l}\left(\pi_{k-r+1}^{\perp} L \pi_{k-r+1}^{\perp}\right) \tilde{\pi}_{j}\right| \leqq c \delta^{-i-j} .
$$

Thus

$$
\left|\tilde{\pi}_{i}\left(\pi_{k-r+1}^{\perp} L \pi_{k-r+1}^{\perp}\right)^{-1} \tilde{\pi}_{k-r} v\right| \leqq c \delta^{-(k-r)-i}
$$

and

$$
\left|\sum_{m} \sum_{l} \tilde{\pi}_{l}\left(\pi_{k-r+1}^{\perp} l \pi_{k-r+1}^{\perp}\right)^{-1} \tilde{\pi}_{m} L \tilde{\pi}_{l} \omega\right| \leqq \sum_{m} \sum_{l} c \delta^{-i-m}\left|\tilde{\pi}_{m} L \tilde{\pi}_{l}\right|\left|\tilde{\pi}_{l} \omega\right| .
$$

Since $l>k-r$, it follows by the induction on $\max \{i, j\}$ that

$$
\left|\tilde{\pi}_{l} \omega\right|=\left|\tilde{\pi}_{l}\left(\pi_{k}^{\perp} L \pi_{k}^{\perp}\right)^{-1} \tilde{\pi}_{k-r} v\right| \leqq c \delta^{-l-(k-r)} .
$$

From Lemma 5.12 , since $l \geqq k-r+1 \geqq 2$ and $l \geqq k-r+1>m$,

$$
\left|\tilde{\pi}_{m} L \tilde{\pi}_{l}\right| \in O\left(\delta^{m+l+1}\right) .
$$

Substituting (5.26) and (5.27) into (5.25) yields

$$
\left|\sum_{m} \sum_{l} \tilde{\pi}_{i}\left(\pi_{k-r+1}^{\perp} L \pi_{k-r+1}^{\perp}\right)^{-1} \tilde{\pi}_{l} \omega\right| \leqq c \delta^{-(k-r)-i+1} .
$$

Combining (5.23), (5.24) and (5.28) yields the desired inequality.

$\mathbf{i}=\mathbf{j}=\mathbf{0}$ : The only case not previously covered is $i=j=0$. Suppose $v \in \tilde{E}_{0},|v|=1$ and

$$
\left(\pi_{k}^{\perp} L \pi_{k}^{\perp}\right)^{-1} v=w
$$

so that $w \in E_{k}^{\perp}$ and

$$
\pi_{k}^{\perp} L \pi_{k}^{\perp} w=v
$$


Then

$$
\langle L w, w\rangle=\left\langle{ }_{k}^{\perp} L \pi_{k}^{\perp} w, w\right\rangle \leqq\left|\tilde{\pi}_{0} w\right| .
$$

On the other hand, we have

$$
\langle L w, w\rangle=\left\langle L \pi_{2}^{\perp} w, \pi_{2}^{\perp} w\right\rangle+\sum_{\substack{l, j \\ \max \{l, J\} \geqq 2}}\left\langle L \tilde{\pi}_{l} w, \tilde{\pi}_{j} w\right\rangle .
$$

If $\max \{i, j\} \geqq 2$

$$
\left|\left\langle L \tilde{\pi}_{i} w, \tilde{\pi}_{j} w\right\rangle\right|=\left|\left\langle\left(\tilde{\pi}_{j} L, \tilde{\pi}_{i}\right) \tilde{\pi}_{i} w, \tilde{\pi}_{j} w\right\rangle\right| \leqq\left|\tilde{\pi}_{j} L \tilde{\pi}_{i}\right| \mid \tilde{\pi}_{j} w \| .
$$

From Lemma 5.12,

$$
\left|\tilde{\pi}_{j} L \tilde{\pi}_{l}\right| \leqq c \delta^{i+j}
$$

By the downward induction on $\max \{i, j\}$

$$
\left|\tilde{\pi}_{l} w\right|=\left(\tilde{\pi}_{i}\left(\pi_{l}\left(\pi_{k-1} L \pi_{k-1}\right)^{-1} \pi_{0}\right) v \mid \leqq c \delta^{-l}\right.
$$

Similarly

$$
\left|\tilde{\pi}_{j} w\right| \leqq c \delta^{-j}
$$

Thus, there is a $c_{1}$ such that if $\max \{i, j\} \geqq 2$,

$$
\left|\left\langle L \tilde{\pi}_{i} w, \tilde{\pi}_{j} w\right\rangle\right| \leqq c_{1} .
$$

From Lemma 5.4 there is a $c$ such that

$$
\left\langle\pi_{2}^{\perp} w, \pi_{2}^{\perp} w\right\rangle \geqq \frac{1}{2}\left\langle\square^{1,0} \pi_{2}^{\perp} w, \pi_{2}^{\perp} w\right\rangle-c \delta^{2}\left|\pi_{2}^{\perp} w\right|^{2} .
$$

From (H2)

$$
\left\langle\square^{1,0} \pi_{2}^{\perp} w, \pi_{2}^{\perp} w\right\rangle c\left|\tilde{\pi}_{0} w\right|^{2}
$$

Moreover

$$
\left.c \delta^{2} \pi_{2}^{\perp} w\right|^{2}=c \delta^{2}\left|\tilde{\pi}_{0} w\right|^{2}+c \delta^{2}\left|\tilde{\pi}_{1} w\right|^{2} .
$$

From the downward induction on $\max \{i, j\}$ there is a $c$ such that

$$
\left|\tilde{\pi}_{1} w\right|=\tilde{\pi}_{1}\left(\pi_{k-1} L \pi_{k-1}\right)^{-1} \pi_{0} v \mid \leqq c \delta^{-1} .
$$

Thus for $\delta$ small enough

$$
\left\langle L \pi_{2}^{\perp} w, \pi_{2}^{\perp} w\right\rangle \geqq c_{2}\left|\tilde{\pi}_{0} w\right|^{2}-c_{3} .
$$

From (5.29), (5.30), (5.31) and (5.32) there is a constant $c_{4}$ such that

$$
c_{2}\left|\tilde{\pi}_{0} w\right|^{2}-c_{4} \leqq\left|\tilde{\pi}_{0} w\right| \text {. }
$$

which implies there is a $c_{5}$ with

$$
\left|\tilde{\pi}_{0} w\right|=\left|\tilde{\pi}_{0}\left(\pi_{k-1}\right) \tilde{\pi}_{0} v\right| \leqq c_{5}
$$

as desired.

Proof of Part (b). Assume that (a) (b) and (c) have been proved for $k-1$ (where $k \geqq 3)$. Suppose $v \in E_{k, \delta}^{\perp},|v|=1$ and 


$$
\langle L v, v\rangle \leqq c_{1} \delta^{2(k-1)} .
$$

Write $v=\alpha+\beta$ with $\alpha \in E_{k-1, \delta}^{\perp}, \beta \in \widetilde{E}_{k-1, \delta}$. Then

$$
\begin{aligned}
c_{1} \delta^{2(k-1)} & \geqq\langle L v, v\rangle=\langle L \alpha, \beta\rangle+\langle\beta, \beta\rangle \\
& \geqq\langle L \alpha, \alpha\rangle+2\langle L \alpha, \beta\rangle \\
& \geqq\left|L^{\frac{1}{2}} \alpha\right|^{2}-2\left|L^{\frac{1}{2}} \alpha\right|\left|L^{\frac{1}{2}} \beta\right| .
\end{aligned}
$$

By the definition of $\widetilde{E}_{k-1, \delta}$,

$$
\left|L^{\frac{1}{2}} \beta\right|=\left(\left|d_{\delta} \beta\right|^{2}+\left.d_{\delta}^{*} \beta\right|^{2}\right)^{\frac{1}{2}} \in O\left(\delta^{k-1}\right) .
$$

Since $\widetilde{E}_{k-1, \beta}$ is dinite dimensional for $k \geqq 3$ there is a $c_{2}>0$ such that

$$
\left|L^{\frac{1}{2}} \beta\right| \leqq c_{2} \delta^{k-1}
$$

Therefore

$$
c_{1} \delta^{2(k-1)} \geqq\left|L^{\frac{1}{2}} \alpha\right|^{2}-2 c_{2} \delta^{k-1}\left|L^{\frac{1}{2}} \alpha\right|^{2},
$$

which implies there is a $c_{3}$ such that

$$
\left|L^{\frac{1}{2}} \alpha\right| \leqq c_{3} \delta^{k-1}
$$

That is

$$
\langle L \alpha, \alpha\rangle \leqq c_{3}^{2} \delta^{2(k-1)}
$$

By induction on part (a)

$$
\langle L \alpha, \alpha\rangle \leqq c_{4} \delta^{2(k-2)}|\alpha|^{2}
$$

Together, (5.33) and (5.34) imply

$$
|\alpha|^{2} \leqq \frac{c_{3}^{2}}{c_{4}} \delta^{2} .
$$

Therefore, $\frac{\alpha}{\delta} \in E_{k-1, \delta}^{\perp}$ satisfies

$$
\left|\frac{\alpha}{\delta}\right| \leqq \frac{c_{3}^{2}}{c_{4}},\left\langle L\left(\frac{\alpha}{\delta}\right), L\left(\frac{\alpha}{\delta}\right)\right\rangle \leqq c_{3}^{2} \delta^{2(k-2)} .
$$

By induction on part (b), for $0 \leqq j \leqq k-2$ there is a $c>0$ s.t.

$$
\left|\tilde{\pi}_{i}\left(\frac{\alpha}{\delta}\right)\right| \leqq c \delta^{(k-1)-i-1} .
$$

Therefore

$$
\left|\tilde{\pi}_{i, \delta} v\right|=\left|\tilde{\pi}_{i, \delta} \alpha\right| \leqq c \delta^{k-i-1}
$$

for $0 \leqq i \leqq k-2$. For $i=k-1$, the estimate follows from $\left|\tilde{\pi}_{k-1} v\right| \leqq|v|=1$. This proves part (b).

Proof of Part (a). We assume (a), (b), (c) have been proved $k-1$, and part (b) has been proved for $k(\geqq 3)$. We will show that, restricted to $E_{k, \delta}^{\perp}$, 


$$
\left|\left(\pi_{k}^{\perp} L \pi_{k}^{\perp}\right)^{-1}\right| \leqq c \delta^{-2(k-1)} .
$$

Let $\lambda_{\delta}$ be the smallest eigenvalue of $\pi_{k}^{\perp} L \pi_{k}^{\perp}$ restricted to $E_{k, \delta}^{\perp}$. Since

$$
\langle L v, v\rangle \in O\left(\delta^{2(k-1)}\right)
$$

for $v \in \widetilde{E}_{k-1}, \lambda_{\delta} \in O\left(\delta^{2(k-1)}\right)$. Thus if $w$ is the corresponding eigenfunction, $|w|=1$, then by part (b)

$$
\left|\left(1-\tilde{\pi}_{k-1}\right) w\right| \in O(\delta)
$$

Now we have

$$
\begin{aligned}
\lambda_{\delta}^{-1} & =\left\langle\left(\pi_{k}^{\perp} L \pi_{k}^{\perp}\right)^{-1} w, w\right\rangle \\
& =\left\langle\left(\pi_{k}^{\perp} L \pi_{k}^{\perp}\right)^{-1} w, \tilde{\pi}_{k-1} w\right\rangle+O(\delta) \lambda_{\delta}^{-1} \\
& =\left\langle w,\left(\pi_{k}^{\perp} L \pi_{k}^{\perp}\right)^{-1} \tilde{\pi}_{k-1} w\right\rangle+O(\delta) \lambda_{\delta}^{-1} \\
& =\left\langle\tilde{\pi}_{k-1} w,\left(\pi_{k}^{\perp} L \pi_{k}^{\perp}\right)^{-1} \tilde{\pi}_{k-1} w\right\rangle+O(\delta)\left|\left(\pi_{k}^{\perp} L \pi_{k}^{\perp}\right)^{-1} \tilde{\pi}_{k-1} w\right|+O(\delta) \lambda_{\delta}^{-1} .
\end{aligned}
$$

Note that

$$
\left|\left(\pi_{k}^{\perp} L \pi_{k}^{\perp}\right)^{-1} \tilde{\pi}_{k-1} w\right| \leqq \lambda_{\delta}^{-1}\left|\tilde{\pi}_{k-1} w\right|+\lambda_{\delta}^{-1}(1+O(\delta)) .
$$

Therefore, (5.35) implies

$$
\left\langle\tilde{\pi}_{k-1} w,\left(\pi_{k}^{\perp} L \pi_{k}^{\perp}\right)^{-1} \tilde{\pi}_{k-1} w\right\rangle=\lambda_{\delta}^{-1}(1+O(\delta)) .
$$

The desired estimate on $\lambda_{\delta}^{-1}$ follows once we see that

$$
\left|\tilde{\pi}_{k-1}\left(\pi_{k}^{\perp} L \pi_{k}^{\perp}\right)^{-1} \tilde{\pi}_{k-1}\right| \leqq c \delta^{-2(k-1)} .
$$

Writing $E_{k, \delta}^{\perp}=E_{k-1, \delta}^{\perp} \oplus \widetilde{E}_{k-1, \delta}$ we deompose $\pi_{k}^{\perp} L \pi_{k}^{\perp}$ into a $2 \times 2$ matrix of operators

$$
\pi_{k}^{\perp} L \pi_{k}^{\perp}=\left(\begin{array}{ll}
\pi_{k-1}^{\perp} L \pi_{k-1}^{\perp} & \pi_{k-1}^{\perp} L \tilde{\pi}_{k-1}^{\perp} \\
\tilde{\pi}_{k-1} L \pi_{k-1}^{\perp} & \tilde{\pi}_{k-1} L \tilde{\pi}_{k-1}
\end{array}\right)
$$

Then we can write

$$
\tilde{\pi}_{k-1}\left(\pi_{k}^{\perp} L \pi_{k}^{\perp}\right)^{-1} \tilde{\pi}_{k-1}=\left(\tilde{\pi}_{k-1} L \tilde{\pi}_{k-1}-\tilde{\pi}_{k-1} L \pi_{k}^{\perp}\left(\pi_{k-1}^{\perp} L \pi_{k-1}^{\perp}\right)^{-1} \pi_{k-1}^{\perp} L \tilde{\pi}_{k-1}\right)^{-1} .
$$

From Lemma 5.11 , there is a $c>0$ with

$$
\tilde{\pi}_{k-1} L \tilde{\pi}_{k-1} \geqq c \delta^{2(k-1)} .
$$

On the other hand

$$
\tilde{\pi}_{k-1} L \pi_{k-1}^{\perp}\left(\pi_{k-1}^{\perp}\right)^{-1} \pi_{k-1}^{\perp} L \tilde{\pi}_{k-1}=\sum_{i, j=-1}^{k-2} \tilde{\pi}_{k-1} L \tilde{\pi}_{l}\left(\pi_{k-1}^{\perp} L \pi_{k-1}^{\perp}\right)^{-1} \tilde{\pi}_{j} L \tilde{\pi}_{k-1} .
$$

Thus, applying Lemma 5.12 and part (b) for $k-1$, 


$$
\begin{aligned}
& \mid \tilde{\pi}_{k-1} L \pi_{k-1}^{\perp}\left(\pi _ { k - 1 } ^ { \perp } \left(\pi_{k-1}^{\perp} L \pi_{k-1}^{\perp} l \tilde{\pi}_{k-1} \mid\right.\right. \\
& \quad \leqq \sum_{i, j=-1}^{k-2}\left|\tilde{\pi}_{k-1} L \tilde{\pi}_{l}\left(\pi_{k-1}^{\perp} L \pi_{k-1}^{\perp}\right)^{-1} \tilde{\pi}_{j} L \tilde{\pi}_{k-1}\right| \\
& \quad \leqq \sum_{l, j}\left|\tilde{\pi}_{k-1} L \tilde{\pi}_{i}\right|\left|\tilde{\pi}_{i}\left(\pi_{k-1}^{\perp} L \pi_{k-1}^{\perp}\right)^{-1} \tilde{\pi}_{j}\right|\left|\tilde{\pi}_{j} L \tilde{\pi}_{k-1}\right| \\
& \quad \leqq c_{1} \sum_{i, j} \delta^{i+k-1+1} \cdot \delta^{-i-j} \cdot \delta^{j+k-1+1} \\
& \quad \leqq c_{2} \delta^{2(k-1)+2} .
\end{aligned}
$$

Combining (5.36), (5.37) and (5.38) we see that for $\delta$ small enough,

$$
\tilde{\pi}_{k-1}\left(\pi_{k}^{\perp} L \pi_{k}^{\perp}\right)^{-1} \tilde{\pi}_{k-1}=\left(\tilde{\pi}_{k-1} L \tilde{\pi}_{k-1}\left(1+O\left(\delta^{2}\right)\right)\right)^{-1} .
$$

Now $\tilde{\pi}_{k-1} L \tilde{\pi}_{k-1} \geqq c \delta^{2(k-1)}$ implies

$$
\mid \tilde{\pi}_{k-1}\left(\pi_{k}^{\perp}\left(\pi_{k}^{\perp}\right)^{-1} \tilde{\pi}_{k-1} \mid \leqq c^{-1} \delta^{-2(k-1)},\right.
$$

is desired.

This theorem provides enough information for us to deduce the desired results.

\section{Corollary 5.14 .}

$$
\begin{aligned}
\#\left\{\lambda_{i}(\delta)\right. & \left.\in \operatorname{spec} \square_{\delta}^{p} \mid \liminf _{\delta \rightarrow 0} \delta^{-2 k} \lambda_{i}(\delta)=0\right\} \\
& =\#\left\{\lambda_{i}(\delta) \in \operatorname{spec} \square \square_{\delta}^{p} \mid \lambda_{i}(\delta) \in O\left(\delta^{2 k+2}\right)\right\}=\operatorname{dim} E_{k+1}^{p} .
\end{aligned}
$$

Proof. The first quantity is clearly $\geqq$ the second, is $\geqq$ the third, by (1.8). If either of these inequalities were strict, we could find a sequence $\delta_{i} \rightarrow 0$ and a sequence $\omega_{i} \in \Omega^{p}$ such that $\left|\omega_{i}\right|=1, \omega \perp E_{k+1, \delta}^{p}$, and for every $c>0$,

$$
\left\langle L_{\delta_{l}} \omega_{i}, \omega_{l}\right\rangle<c \delta^{2 k}
$$

for $\delta$ small enough. This contradicts Theorem 5.13 (a).

Now write $\lambda_{i}(\delta) \sim \delta^{k}$ if

$$
\left.\lambda_{i}(\delta) \in O\left(\delta^{k}\right) \text { and }\left(\lambda_{i}(\delta)\right)\right)^{-1} \in O\left(\delta^{-k}\right) .
$$

\section{Theorem 5.15.}

$$
\#\left\{\lambda_{l}(\delta) \in \operatorname{spec} \square \delta \mid \lambda_{l}(\delta) \sim \delta^{2 k}\right\}=\operatorname{dim} \widetilde{E}_{k}^{p}
$$

Proof. From Corollary 5.14

$$
\#\left\{\lambda_{i}(\delta) \in O\left(\delta^{2 k}\right)\right\}=\operatorname{dim} E_{k}^{p} .
$$

Moreover,

$$
\left(\lambda_{i}(\delta)\right)^{-1} \notin O\left(\delta^{-2 k}\right) \leftrightarrow \delta^{2 k}\left(\lambda_{i}(\delta)\right)^{-1} \notin O(1) \leftrightarrow \liminf _{\delta \rightarrow 0} \delta^{-2 k} \lambda_{i}(\delta)=0
$$


From Corollary 5.14, all eigenvalues $\lambda_{i}$ with

$$
\left(\lambda_{i}(\delta)\right)^{-1} \notin O\left(\delta^{2 k}\right)
$$

are $O\left(\delta^{2 k+2}\right)$ and the number of such eigenvalues is $\operatorname{dim} E_{k+1}^{p}$.

Thus

$$
\begin{aligned}
\#\left\{\lambda_{l}(\delta) \sim O\left(\delta^{2 k}\right)\right\} & =\#\left\{\lambda_{l}(\delta) \in O\left(\delta^{2 k}\right)\right\}-\#\left\{\lambda_{l}(\delta) \in O\left(\delta^{2 k+2}\right)\right\} \\
& =\operatorname{dim} E_{k}^{p}-\operatorname{dim} E_{k+1}^{p}=\operatorname{dim} \widetilde{E}_{k}^{p} \cdot \square
\end{aligned}
$$

Corollary 5.16. If $\lambda_{i}^{p}(\delta) \in O\left(\delta^{k}\right)$ for all $k$, then $\lambda_{i}^{p}(\delta) \equiv 0$ for all $\delta$.

Proof. Clearly $\left\{\lambda_{i}(\delta) \in O\left(\delta^{k}\right)\right.$ for all $\left.\delta\right\} \supseteq\left\{\lambda_{l}(\delta) \equiv 0\right.$ for all $\left.\delta\right\}$. From Corollary $5.14 \#\left\{\lambda_{i}^{p}(\delta) \in O\left(\delta^{k}\right)\right.$ for all $\left.k\right\}=\operatorname{dim} E_{N}^{p}=\operatorname{dim} E_{\infty}^{p}$. In addition, by the results of Sect. 3,

$$
\begin{aligned}
\operatorname{dim} E_{N}^{p} & =\operatorname{dim} H^{p}(M, E) \\
& =\#\left\{\lambda_{l}^{p}(\delta) \equiv 0 \text { for all } \delta\right\} .
\end{aligned}
$$

This proves the corollary.

We now investigate the behavior of the eigenspaces as $\delta \rightarrow 0$. Let

$$
\operatorname{eig}_{k, \delta}^{p}=\operatorname{span}\left\{\omega_{i}(\delta)=\lambda_{l}(\delta) \omega_{l}(\delta) \text { with } \lambda_{l}(\delta) \in\left(\delta^{2 k}\right)\right\}
$$

Theorem 5.17. For $k>1$,

$$
\operatorname{eig}_{k, \delta}^{p}=E_{k}^{p}+O(\delta)
$$

By which we mean, if $v_{\delta} \in \operatorname{eig}_{k, \delta}^{p},\left|v_{\delta}\right|=1$, then we can write

$$
v_{\delta}=\alpha_{\delta}+\beta_{\delta}
$$

with $\alpha_{\delta} \in E_{k}^{p}$, and $|\beta| \in O(\delta)$. Equivalently, if $\rho_{k, \delta}$ is the orthogonal projection onto $\operatorname{eig}_{k, \delta}^{p}$ then

$$
\left|\rho_{k . \delta}-\pi_{k}\right| \in O(\delta)
$$

Proof. If $v_{\delta} \in \operatorname{eig}_{k, \delta}^{p},|v-\delta|=1$ then

$$
\left\langle L_{\delta} v_{\delta}, v_{\delta}\right\rangle \leqq c_{1} \delta^{2 k}
$$

Write $v_{\delta}=\alpha_{\delta}+\beta_{\delta}$ with

$$
\alpha_{\delta} \in E_{k, \delta}^{p}, \quad \beta_{\delta} \in\left(E_{k, \delta}^{p}\right)^{\perp}
$$

Then

$$
\langle L v, v\rangle=\langle L \alpha, \alpha\rangle+2\langle L \alpha, \beta\rangle+\langle L \beta, \beta\rangle=\left|L^{\frac{1}{2}} \alpha\right|^{2}+2\left\langle L^{\frac{1}{2}} \alpha, L^{\frac{1}{2}} \beta\right\rangle+\left.L^{\frac{1}{2}} \beta\right|^{2} .
$$

Since $k>1$, there is a $c>0$ with

$$
\left|L^{\frac{1}{2}} \alpha\right| \leqq c \mid \delta^{k} \leqq c_{2} \delta^{k}
$$

so that

$$
\left|L^{\frac{1}{2}} \beta\right|^{2}-2 c_{2} \delta^{k}\left|L^{\frac{1}{2}} \beta\right| \leqq\left(c_{1}-c_{2}\right) \delta^{2 k},
$$


which implies

$$
\langle L \beta, \beta\rangle=\left|L^{\frac{1}{2}} \beta\right|^{2} \leqq c_{3} \delta^{2 k} .
$$

It follows from theorem 5.13 (a), that $|\beta| \in O(\delta)$. Thus $v_{\delta}=\alpha_{\delta}+O(\delta)$, with $\delta \in$ $R_{k, \delta}^{p}$, but

$$
E_{k, \delta}^{p}=E_{k}^{p}+O(\delta),
$$

which proves the theorem.

The following two corollaries are immediate

Corollary 5.18. Let $\mathscr{H}_{\delta}^{p}(M, V)$ denote the kernel of $\square_{\delta}^{p}$ (=the space of $g_{\delta^{-}}$ harmonic p-forms), then

$$
\rho_{\delta} \mathscr{H}_{\delta}^{p}(M, V)=\operatorname{ker} L_{\delta}^{p}=E_{\infty}^{p}+O(\delta) .
$$

\section{Corollary 5.19. Let}

$$
\widetilde{\operatorname{eig}}_{k, \delta}^{p}=\operatorname{span}\left\{\omega_{i}(\delta) \mid \square_{\delta}^{p} \omega_{l}(\delta)=\lambda_{i}(\delta) \omega_{i}(\delta) \text { with } \lambda_{i}(\delta) \sim \delta^{2 k}\right\},
$$

then

$$
\widetilde{\operatorname{eig}}_{k, \delta}^{p}=\widetilde{E}_{k}^{p}+O(\delta)
$$

We are now ready to make precise statements about the asymptotics of the eigenvalues. We have already seen that

$$
\left\{\lambda_{i}^{p}(\delta) \mid \operatorname{dim} E_{k+1}^{p}+1 \leqq i \leqq \operatorname{dim} E_{k}^{p}\right\}
$$

are the eigenvalues of $L_{\delta}^{p}$ which are $\sim \delta^{2 k}$, and the corresponding eigenspaces converge to $\widetilde{E}_{k}^{p}$. We now prove

Theorem 5.20. Fix $p$. Then for every $i$,

$$
\begin{gathered}
\operatorname{dim} E_{k+1}^{p}+1 \leqq i \leqq \operatorname{dim} E_{k}^{p} \quad \text { if } k>1, \\
\operatorname{dim} E_{2}^{p}+1 \leqq i<\infty \quad \text { if } k=1,
\end{gathered}
$$

we have

$$
\lambda_{i}(\delta)=\delta^{2 k} \bar{\lambda}_{i}+O\left(\delta^{2 k+1}\right)
$$

for some $\bar{\lambda}_{i}$. These $\operatorname{dim} \widetilde{E}_{k}^{p}$ values of $\bar{\lambda}_{i}$ are given by the eigenvalues of the operator

$$
\Delta_{k}^{p}: \widetilde{E}_{k}^{p} \rightarrow \widetilde{E}_{k}^{p}
$$

[In particular, for $k>1$,

$$
\left.\left\{\lambda_{l}(\delta) \mid \lambda_{i}(\delta) \sim \delta^{2 k}\right\}=\delta^{2 k}\left\{\text { eigenvalues of } \Delta_{k}^{p}: \widetilde{E}_{k}^{p} \rightarrow \widetilde{E}_{k}^{p}\right\}+O\left(\delta^{2 k+1}\right)\right] .
$$

Proof. Assume $k>1$. First we prove that the eigenvalues of $L_{\delta}^{p}$ which are $\sim \delta^{2 k}$ are closely approximated by the eigenvalues of $\pi_{k+1, \delta}^{\perp} L_{\delta} \pi_{k+1, \delta}^{\perp}$. If $v \in \Omega^{p},|v|=1$ then write

$$
v=\alpha+\beta
$$

with

$$
\alpha \in\left(E_{k+1, \delta}^{p}\right)^{\perp}, \quad \beta \in E_{k+1, \delta}^{p} .
$$


Then

$$
\langle L v, v\rangle=\langle L \alpha, \alpha\rangle+2\left\langle L^{\frac{1}{2}} \alpha, L^{\frac{1}{2}} \beta\right\rangle+\langle L \beta, \beta\rangle .
$$

Note that

$$
\left|2\left\langle L^{\frac{1}{2}} \alpha, L^{\frac{1}{2}} \beta\right\rangle\right| \leqq \delta\langle L \alpha, \alpha\rangle+\delta^{-1}\langle L \beta, \beta\rangle,
$$

and there is a $c$ such that for all $\delta$ and $\beta \in E_{k+1, \delta}^{p}$,

$$
\langle L \beta, \beta\rangle \leqq c \delta^{2 k+2}|\beta|^{2} \leqq c \delta^{2 k+2} .
$$

Thus, since $\pi_{k+1, \delta}^{\perp} v=\alpha$, we have

$$
\langle L v, v\rangle=\left\langle\pi_{k+1}^{\perp} L \pi_{k+1}^{\perp} v, v\right\rangle+c_{1}\left\langle\pi_{k+1}^{\perp} L \pi_{k+1}^{\perp} v, v\right\rangle+c_{2},
$$

where

$$
\left|c_{1}\right| \leqq \delta,\left|c_{2}\right| \leqq c \delta^{2 k+1} .
$$

Let $\mu_{1}(\delta) \leqq \mu_{2}(\delta) \leqq \cdots$ be the eigenvalues of $\pi_{k+1}^{\perp} L^{p} \pi_{k+1}^{\perp}$, so that, in particular,

$$
\mu_{1}(\delta) \equiv \mu_{2}(\delta) \equiv \cdots \equiv \mu_{\mathrm{d} \mathrm{m} E_{k+1}^{p}}(\delta) \equiv 0 .
$$

Then (5.39) implies that for all $i$

$$
\left|\lambda_{l}-\mu_{l}\right| \leqq c\left(\delta \mu_{l}+\delta^{2 k+1}\right) .
$$

This shows that for

$$
\operatorname{dim} E_{k+1}^{p}+1 \leqq i \leqq \operatorname{dim} E_{k}^{p}
$$

the eigenvalues $\mu_{l}(\delta)$ are $\sim \delta^{2 k}$, and for such $i$

$$
\left|\lambda_{l},-\mu_{\imath}\right| \leqq c \delta^{2 k+1}
$$

The theorem follows from proving that these $\mu_{l}$ 's have the form

$$
\mu_{l}=\delta^{2 k} \bar{\mu}_{i}+O\left(d^{2 k+1}\right)
$$

with the $\bar{\mu}_{l}$ 's the eigenvalues of $\Delta_{k}^{p}$. The inverses of the non-zero $\mu_{l}$ 's are given by the eigenvalues of $\left(\pi_{k+1}^{\perp} L \pi_{k+1}^{\perp}\right)^{-1}$ restricted to $\left(E_{k}^{p}\right)^{\perp}$. From Theorem 5.13 (c) it follows that, restricted to $\left(E_{k+1}^{p}\right)^{\perp}$

$$
\left|\left(\pi_{k+1}^{\perp} L \pi_{k+1}^{\perp}\right)^{-1}-\tilde{\pi}_{k}\left(\pi_{k+1}^{\perp} L \pi_{k+1}^{1}\right)^{-1} \tilde{\pi}_{k}\right| \leqq c_{1} \delta^{-2 k+1} .
$$

Thus, if $v_{1}(\delta), \ldots, v_{\operatorname{dim}} \widetilde{E}_{k}^{p}(\delta)$ are the inverses of the non-zero eigenvalues of $\tilde{\pi}_{k}\left(\pi_{k+1}^{\perp} L \pi_{k+1}^{\perp}\right)^{-1} \tilde{\pi}_{k}$, we have, for $1 \leqq i \leqq \operatorname{dim} \widetilde{E}_{k}^{p}$,

$$
\left|\mu_{\operatorname{dim} E_{k+1}+l}(\delta)-v_{i}(\delta)\right| \leqq c_{2} \delta^{2 k+1} .
$$

From (5.36) we see that

$$
\left(\tilde{\pi}_{k}\left(\pi_{k+1}^{\perp} L \pi_{k+1}^{\perp}\right)^{-1} \tilde{\pi}_{k}\right)^{-1}=\tilde{\pi}_{k} L \tilde{\pi}_{k}+\tilde{\pi}_{k} L \pi_{k+1}^{\perp}\left(\pi_{k+1}^{\perp} L \pi_{k+1}^{\perp}\right)^{-1} \pi_{k+1}^{\perp} L \tilde{\pi}_{k} .
$$

From (5.19) we have

$$
\tilde{\pi}_{k} L \tilde{\pi}_{k}=\delta^{2 k} \Delta_{k}+O\left(\delta^{2 k+1}\right),
$$


and from Lemma 5.12 combined with Theorem 5.13 (c) we find

$$
\left|\tilde{\pi}_{k} L \pi_{k+1}^{\perp}\left(\pi_{k+1}^{\perp} L \pi_{k+1}^{\perp}\right)^{-1} \pi_{k+1}^{\perp} L \tilde{\pi}_{k}\right| \leqq c_{2} \delta^{2 k+2},
$$

which proves the theorem in the case $k>1$.

If $k=1$ then $\operatorname{dim} E_{k}^{p}=\infty$, and we do not have uniform bounds for the errors which appear. However, this is not a problem. Fix $c>0$ and consider

$$
\Gamma_{c}=\left\{\lambda_{i}(\delta) \mid \limsup _{\delta \rightarrow 0} \delta^{-2} \lambda_{i}(c)<c\right\} .
$$

From Lemma 5.4 we know $L_{\delta}>\delta^{2} k$ for a $2^{\text {nd }}$ order operator $k$ with positive symbol, so there is an upper bound $N_{c}$, independent of $\delta$, for the number of eigenvalues of $L_{\delta}$ which are $<c \delta^{2}$. Then the same argument as for $k>1$ shows that for $\operatorname{dim} E_{2}^{p}+1 \leqq i \leqq \# \Gamma_{c}$,

$$
\lambda_{i}(\delta)=\delta^{2} \bar{\lambda}_{i}+O(\delta)
$$

where the $\bar{\lambda}_{i}$ are the eigenvalues of

$$
\Delta_{0}^{p}: \widetilde{E}_{0}^{p} \rightarrow \widetilde{E}_{0}^{p}
$$

which are $<c$. Letting $c \rightarrow \infty$ completes the proof.

So far, we have shown that the eigenspaces of $L_{\delta}^{p}$ approach the spaces $E_{k}^{p}$ continuously. We complete this section by showing that our previous analysis actually implies the $C^{\infty}$ convergence of the kernel of $L_{\delta}^{p}$.

Theorem 5.21. The spaces

$$
\operatorname{Ker} L_{\delta}^{p}=\rho_{\delta} \mathscr{H}_{\delta}^{p}(M, V)
$$

form a $C^{\infty}$ map from $[0,1]$ to the space of $\left(\operatorname{dim} H^{p}(M, V)\right)$-dimensional subspaces of the $L^{2} p$-forms on $M$.

(Note : This generalizes Theorem 17 of [Ma-Me].)

Proof. Fix $M>0$. We will show that $\rho_{\delta} \mathscr{H}_{\delta}^{p}$ is $C^{M}$. We follow the proof of Theorem 5.17, with one modification. In defining $E_{N, \delta}^{p}=E_{\infty, \delta}^{p}$, we truncated the formal power series $(5.18)$ at the $\delta^{N+2}$ term. To prove $C^{M}$ convergence, we truncate the power series at $\delta^{N+M}$. That is, let

$$
\Phi^{\prime}(v)=v+\delta v_{1}+\cdots+\delta^{N+M-1} v_{N+M-1},
$$

so that

$$
\begin{aligned}
& d_{\delta} \Phi^{\prime}(v) \in \delta^{N+M} \Omega^{p+1}[\delta], \\
& d_{\delta}^{*} \Phi^{\prime}(v) \in \delta^{N+M} \Omega^{p-1}[\delta] .
\end{aligned}
$$

Let $E_{\infty, \delta}^{p}$ denote the image of $\Phi^{\prime}$ applied to the space $E_{\infty}^{p}$. Then $E_{\infty, \delta}^{p}$ is $C^{\infty}$ on $[0,1]$ (in fact is polynomial). Now we continue as before. Suppose

$$
v_{\delta} \in \rho_{\delta} \mathscr{H}_{\delta}^{p},
$$


so that

$$
\left\langle L_{\delta} v_{\delta}, v_{\delta}\right\rangle=0
$$

and

$$
\left|v_{\delta}\right|=1
$$

Write

$$
v_{\delta}=\alpha_{\delta}+\beta_{\delta}
$$

with

$$
\alpha_{\delta} \in E_{\infty, \delta}^{p}, \beta_{\delta} \in\left(E_{\infty, \delta}^{p}\right)^{\perp}
$$

Then

$$
0=\langle L v, v\rangle=\left|L^{\frac{1}{2}} \alpha\right|^{2}+2\left\langle L^{\frac{1}{2}} \alpha, L^{\frac{1}{2}} \beta\right\rangle+\left|L^{\frac{1}{2}} \beta\right|^{2},
$$

so that

$$
\begin{aligned}
0 & \geqq\left|L^{\frac{1}{2}} \beta\right|^{2}+2\left\langle L^{\frac{1}{2}} \alpha, L^{\frac{1}{2}} \beta\right\rangle \\
& \geqq\left|L^{\frac{1}{2}} \beta\right|^{2}-2\left|L^{\frac{1}{2}} \alpha\right|\left|L^{\frac{1}{2}} \beta\right| \\
& =\left|L^{\frac{1}{2}} \beta\right|\left(\left|L^{\frac{1}{2}} \beta\right|-2\left|L^{\frac{1}{2}} \alpha\right|\right) .
\end{aligned}
$$

Therefore

$$
\left|L^{\frac{1}{2}} \beta\right| \leqq 2\left|L^{\frac{1}{2}} \alpha\right|
$$

Now, $\alpha \in E_{\infty, \delta}^{p}$ so from (5.40),

$$
\left|L^{\frac{1}{2}} \alpha\right| \leqq c \delta^{N+M}|\alpha| \leqq c \delta^{N+M} .
$$

Moreover, $\beta \in\left(E_{\infty, \delta}^{p}\right)^{\perp}$ implies there is a $c>0$ such that

$$
\left|L^{\frac{1}{2}} \beta\right| \geqq c \delta^{N-1}|\beta|
$$

This yields

$$
|\beta| \leqq c \delta^{m+1}
$$

Thus

$$
\rho_{\delta} \mathscr{H}_{\delta}^{p}=E_{\infty, \delta}^{p}+O\left(\delta^{M+1}\right),
$$

which implies $\rho_{\delta} \mathscr{H}_{\delta}^{p}$ is $C^{M}$ as desired.

As noted in [Ma-Me], this implies that any formally harmonic power series (5.12) is, in fact, the Taylor series at $\delta=0$ of a $C^{\infty}$ family of forms $\omega_{\delta}$ satisfying,

$$
\text { for every } \delta \in[0,1] \omega_{\delta} \in \rho_{\delta} \mathscr{H}_{\delta}^{p}(M, V) \text {. }
$$

Applying the map $\rho_{\delta}^{-1}$, we learn

Corollary 5.22 (Corollary 18 of [Ma-Me]): The space $H_{\delta}^{p}(M, V)$ defines a $C^{\infty}$ map from $[0,1]$ to the space of $\left(\operatorname{dim} H^{p}(M, V)\right)$-dimensional subspaces of the $L^{2}$ p-forms on $M$. 


\section{References}

[Bi-Fr] Bismut, J.-M., Freed, D.S.: The analysis of Elliptic Families, I. Metrics and Connections on Determinant Bundles. Commun. Math. Phys. 106, 159-176 (1986); II. Dirac Operators Eta Invariants and the Holonomy Theorem. 107, 103-163 (1986)

[Bi-Ch] Bismut, J.-M., Cheeger. J.: $\eta$-Invariants and Their Adiabatic Limits. J. Am. Math. Soc. 2, 33-70 (1989)

[Bo-Tu] Bott, R. Tu, L.W.: Differential Forms in Algebraic Topology. Graduate Texts in Mathematics no. 82, Berlin Heidelberg, New York: Springer, 1982

[Ch] Cheeger, J.: Eta Invariants, the Adiabatic Approximation and Conical Singularities. J. Diff. Geom. 26, 175-221 (1987)

[Dai] Dai, X.: Adiabatic Limits, Non-multiplicity of Signature and the Leray Spectral Sequence.. J. Am. Math. Soc. 4, 265-321 (1991)

[Du-Sc] Dunford, N., Schwartz, J.: Linear Operators Part II. Pure and Applied Mathematics, Vol. VII New York: Interscience Publishers, 1963

[Fo] Forman, R.: Hodge Theory and Spectral Sequences. Topology 33, 591-611 (1994)

[Ma-Me] Mazzeo, R.R., Melrose, R.B.: The Adiabatic Limit, Hodge Cohomology and Leray's Spectral Sequence for a Fibration. J. Diff. Geom. 31, 185-213 (1990)

[McC] McClearly, J.: User's Guide to spectral Sequences. Mathematics Lecture Series no. 12, Berkeley: Publish or Perish, Inc., 1985

[Mo] Molino, P.: Riemannian Foliations, Progress in Mathematics no. 73, Baset, Boston: Birkhäuser, 1988

[Re] Reinhart, B.L.: Differential Geometry of Foliations. Ergebnisse der mathematik und ihrer Grenzgebiete no. 99, Berlin, Heidelberg, New York: Springer, 1983

[Sa] Sarkaria, K.S.: A Finiteness Theorem for Foliated Manifolds. J. Math. Soc. Japan 30, 687-96 (1978)

[Wi] Witten, E.: Global Gravitational Anomalies. Commun. Math. Phys, 100, 197-229 (1985)

Communicated by A. Connes 